\title{
THE DETAILED CHEMICAL PROPERTIES OF M31 STAR CLUSTERS. I. Fe, ALPHA AND LIGHT ELEMENTS*
}

\author{
Janet E. Colucci ${ }^{1,3}$, Rebecca A. Bernstein ${ }^{1}$, and Judith G. Cohen ${ }^{2}$ \\ ${ }^{1}$ The Observatories of the Carnegie Institution for Science, 813 Santa Barbara St., Pasadena, CA 91101, USA; jcolucci@ obs.carnegiescience.edu \\ ${ }^{2}$ Palomar Observatory, Mail Stop 105-24, California Institute of Technology, Pasadena, CA 91125, USA \\ Received 2014 July 18; accepted 2014 October 29; published 2014 December 5
}

\begin{abstract}
We present ages, $[\mathrm{Fe} / \mathrm{H}]$ and abundances of the $\alpha$ elements $\mathrm{Ca}$ I, Si I, Ti I, Ti II, and light elements $\mathrm{Mg}$ I, Na I, and $\mathrm{Al}$ I for 31 globular clusters (GCs) in M31, which were obtained from high-resolution, high signal-to-noise ratio $>60$ echelle spectra of their integrated light (IL). All abundances and ages are obtained using our original technique for high-resolution IL abundance analysis of GCs. This sample provides a never before seen picture of the chemical history of M31. The GCs are dispersed throughout the inner and outer halo, from $2.5 \mathrm{kpc}<R_{\mathrm{M} 31}<$ $117 \mathrm{kpc}$. We find a range of $[\mathrm{Fe} / \mathrm{H}]$ within $20 \mathrm{kpc}$ of the center of $\mathrm{M} 31$, and a constant $[\mathrm{Fe} / \mathrm{H}] \sim-1.6$ for the outer halo clusters. We find evidence for at least one massive GC in M31 with an age between 1 and 5 Gyr. The $\alpha$-element ratios are generally similar to the Milky Way GC and field star ratios. We also find chemical evidence for a late-time accretion origin for at least one cluster, which has a different abundance pattern than other clusters at similar metallicity. We find evidence for star-to-star abundance variations in $\mathrm{Mg}, \mathrm{Na}$, and $\mathrm{Al}$ in the $\mathrm{GCs}$ in our sample, and find correlations of $\mathrm{Ca}, \mathrm{Mg}, \mathrm{Na}$, and possibly $\mathrm{Al}$ abundance ratios with cluster luminosity and velocity dispersion, which can potentially be used to constrain GC self-enrichment scenarios. Data presented here were obtained with the HIRES echelle spectrograph on the Keck I telescope.
\end{abstract}

Key words: galaxies: halos - galaxies: individual (M31) - galaxies: star clusters: general - Local Group stars: abundances

Online-only material: color figures, machine-readable tables

\section{INTRODUCTION}

Study of M31, the Milky Way's (MW) nearest massive neighbor, is interesting for many reasons. One of the most fundamental questions is whether the characteristics of the M31 spiral galaxy support the assertion that the MW is a "normal" spiral galaxy. This is important because we can study the properties of the MW in great detail, and studies of our own galaxy by necessity are the foundation for our understanding of how galaxies in general form and evolve. As the next closest massive galaxy, M31 is the first place to test galaxy formation theories developed from studies of the MW, and in some respects is a more ideal test-case because M31 can be observed as a whole from the outside, whereas study of our own galaxy is complicated by our position within it.

However, the distance to M31 means that we are unable to study its individual stars at the same level of detail that we can obtain in the MW. For example, much of our detailed knowledge of the evolution of the MW has come from chemical evolution studies of our Galaxy's individual stars. Stars are ideal records of chemical evolution because their atmospheres generally retain the same chemical composition as the gas reservoir out of which they formed, and therefore with "fossil" chemistry of stars of all ages, one can gain unparalleled insight on the history of a galaxy. The most precise detailed chemical abundance analyses require high-resolution, high signal-to-noise ratio $(\mathrm{S} / \mathrm{N})$ spectra, so that individual transitions of a myriad of elements can be isolated and analyzed. Unfortunately, at a distance of $785 \mathrm{kpc}$

\footnotetext{
* The data presented herein were obtained at the W. M. Keck Observatory, which is operated as a scientific partnership among the California Institute of Technology, the University of California and the National Aeronautics and Space Administration. The Observatory was made possible by the generous financial support of the W. M. Keck Foundation.

3 NSF Astronomy and Astrophysics Postdoctoral Fellow.
}

(McConnachie et al. 2005), the individual stars in M31 are far too faint for obtaining high-resolution spectra.

With the development of our original technique for abundance analysis of high-resolution integrated light (IL) spectra of globular clusters (GCs), we can now make significant advances in chemical evolution studies of distant massive galaxies. Unresolved GCs, which are luminous and therefore observationally accessible to large distances, can be used to learn about the chemical enrichment and formation history of other galaxies, just as they were originally used to learn about the formation of the MW (e.g., Eggen et al. 1962; Searle \& Zinn 1978). Our technique has been developed and demonstrated on resolved GCs in the MW and Large Magellanic Cloud (LMC) in a series of papers: Bernstein \& McWilliam (2005), McWilliam \& Bernstein (2008, hereafter MB08), Colucci et al. (2011, hereafter C11), and Colucci \& Bernstein (2012). These works demonstrate that the IL analysis provides accurate $\mathrm{Fe}$ abundances and $[\mathrm{X} / \mathrm{Fe}]$ ratios to $\sim 0.1 \mathrm{dex}$, as well as distinguishes ages for $\mathrm{GCs}$ with a range in properties, including $[\mathrm{Fe} / \mathrm{H}]$ of -2 to +0 and ages from 0.05 to $12 \mathrm{Gyr}$. We also note that a detailed discussion of potential systematic errors in high-resolution IL analysis was presented for a similar technique in Sakari et al. (2014). This work also demonstrated that systematic uncertainties in GC IL analysis are small for $\mathrm{Fe}, \mathrm{Ca}, \mathrm{Ti}$, and $\mathrm{Ni}$, although individual elements with few transitions (Ba II and Eu II) can have larger systematic uncertainties.

With this method, we have now begun an unprecedented study of the chemical composition of the GC system of M31. Presently, the number of confirmed, massive GCs in M31 is $>400$; a long history of study of M31 GCs is embodied in the extensive photometric and spectroscopic properties maintained in the Revised Bologna Catalog (Galleti et al. 2004). With the recent addition of a large imaging survey of the outer halo of M31, the Pan-Andromeda Archaeological Survey (PAndAS; 
McConnachie et al. 2009; Ibata et al. 2014), the GC system out to projected galactocentric radii of $\sim 150 \mathrm{kpc}$ is thought to be complete to cluster magnitudes of $M_{V}=-6$ (Huxor et al. 2014). The inner GCs of M31 have been well-studied with low-resolution spectroscopy; most GCs have several metallicity estimates available from a variety of methods (e.g., Huchra et al. 1991; Barmby \& Huchra 2000; Perrett et al. 2002; Beasley et al. 2005; Puzia et al. 2005; Galleti et al. 2009; Caldwell et al. 2011). As part of our ongoing project, we present the first detailed chemical abundances of GCs in M31, which now allows us to compare the detailed chemical history of old stellar populations in M31 to those in the MW for the first time.

Detailed abundances of $\sim 20$ elements were presented for a pilot sample of 5 M31 GCs in Colucci et al. (2009, hereafter C09). Here we extend the sample of $\mathrm{C} 09$ and now present ages and abundances of $\mathrm{Fe}, \mathrm{Ca}, \mathrm{Ti}, \mathrm{Si}, \mathrm{Mg}, \mathrm{Na}$, and $\mathrm{Al}$ of an additional $26 \mathrm{GCs}$ in M31. In future papers we will present detailed abundances of Fe-peak and r- and s-process elements in this sample of GCs. In Section 2, we describe the target selection, observations, data reduction and velocity measurements. In Section 3, we describe the equivalent width (EW) and line synthesis abundance analyses of Fe I lines, which are used to determine both $[\mathrm{Fe} / \mathrm{H}]$ and age. In Section 4, we present the results for Fe II, Ca I, Si I, Ti I, Ti II, Na I, Al I, and Mg I; and in Section 5, we discuss the results with respect to the star formation history of M31, formation histories of GCs in general, and previous work on the GC system of M31.

\section{TARGETS, OBSERVATIONS AND REDUCTIONS}

Our GC targets were chosen from the Revised Bologna Catalog (Galleti et al. 2004), and have all been previously spectroscopically confirmed as members of the M31 GC system. Our selection criteria required that the GCs be more luminous than $V \sim 17$ mag, but less luminous than $V \sim 15$ mag because the brightest clusters have the highest velocity dispersions $\left(v_{\sigma}\right)$, leading to more line broadening and blending and are thus more difficult to analyze. We picked GCs that are in relatively uncrowded regions and that are not projected onto the highest surface brightness part of the M31 bulge or disk. While our sample is obviously not complete, we have selected GCs with a wide range in previously estimated $[\mathrm{Fe} / \mathrm{H}]$, age, $v_{\sigma}$, and projected galactocentric distance from M31 $\left(R_{\mathrm{M} 31}\right)$ in order to increase our chances of surveying the range of properties present in M31 GCs. The magnitudes and spatial information are listed for all of the GCs in Table 1. In Figure 1, we show the locations of the GCs in our sample over a composite STScI Digitized Sky Survey image of M31 and the surrounding field. Note that the three GCs with the largest projected distances from M31-B514, MCGC5 and MGC1 - are beyond the edges of the field, which emphasizes the extensive radial coverage of our sample.

We obtained high-resolution IL spectra of the M31 GCs using the HIRES spectrograph (Vogt et al. 1994) on the Keck I telescope. The data were taken over several observing runs from 2008 to 2012. In all observing runs, we used identical setups that utilized the D3 decker, which has a slit size of $1^{\prime \prime} .7 \times 7^{\prime \prime} .0$ and spectral resolution of $R=24,000$, which is sufficient to resolve individual spectral lines of GCs with $v_{\sigma} \geqslant 7 \mathrm{~km} \mathrm{~s}^{-1}$. Twenty-two of the 26 GCs have previously measured half light radii (Barmby et al. 2007; Peacock et al. 2010; Ma et al. 2012; Wang \& Ma 2013) that are between $\sim 0$.'6 and 1". 1 , which means that $70 \%-90 \%$ of the GC light was included in the $1^{\prime \prime} .7 \times 7$ ". 0 slit during the observation. We assume that the other 4 GCs have similar half light radii, with the conclusion that the
GC populations are well sampled in the IL. The wavelength coverage of the HIRES spectra is approximately 3800-8300. Total exposure times were between one and six hr for each GC and are listed in Table 1 along with the date each GC was observed. The total exposure for each GC was divided into 1800 or $3600 \mathrm{~s}$ increments to aid in cosmic ray removal. The $\mathrm{S} / \mathrm{N}$ estimates at $6000 \AA$ are also given in Table 1 . Data were reduced with standard flat fielding, sky subtraction, and wavelength calibration routines in the HiRes Redux pipeline. ${ }^{4}$ To remove the blaze function of HIRES, we used low-order polynomial fits to the spectrum of a $G$ star taken during each run, which should approximately have the same color as the IL of GCs (MB08). In Figures 2-4, we show a portion of the final spectra of the GCs in a $\sim 100 \AA$ region centered at approximately $6365 \AA$, which is a region that includes several spectral features used in the abundance determinations. The GCs are shown in order of increasing metallicity from our analysis, and velocity dispersion of each GC are noted for reference.

\subsection{Velocity Dispersion Measurements}

One-dimensional velocity dispersion $\left(v_{\sigma}\right)$ measurements were obtained by cross correlation with Galactic template stars, as described in C09. In brief, the IRAF task fxcor is used to cross correlate the GC spectra with a template star on an order by order basis. The FWHM of the cross correlation peaks is then converted to a line-of-sight velocity dispersion using an empirical relation, as described in Tonry \& Davis (1979). The template stars used in this analysis include HD188510 (G5V), HR6757 (G8II), HR6940 (G8II-III), and HR7325 (G9III), and were observed with identical setups as the GC targets during each run. The variation in derived velocity dispersion when different template stars were used was generally less than $1-2 \mathrm{~km}$ $\mathrm{s}^{-1}$, which is comparable to the scatter in measurements between individual echelle orders for individual template stars. For our final measurements, we average the results for all four template stars, with an uncertainty equal to the standard deviation of the mean. The results are listed in Table 2, along with the heliocentric corrected radial velocities. In Table 2, we also list previously measured radial velocities and velocity dispersions for the GCs. The majority of GCs with previously measured radial velocities agree with our results to within $3 \sigma$ of the quoted errors.

Nearly all of the previously measured velocity dispersions agree with our results to within $1 \sigma$, and all agree to within $2 \sigma$. We note that some differences between analyses are expected due to differences in apertures, but we neglect that effect here because our primary goal in measuring velocity dispersions is to use them in spectral synthesis analysis. Further discussion of the M31 GC velocity dispersions and an analysis of massto-light $(M / L)$ ratios will be presented in J. E. Colucci et al. (2014, in preparation). For nine of the GCs, we present the first measurements of the velocity dispersion (B034, B311, B312, B383, B403, B457, B514, G327, and MGC1). In Figure 5, we show our velocity dispersion measurements for the M31 GCs as a function of the reddening corrected absolute $V$ magnitudes of the GCs. In this work we use the $V$ and $E(B-V)$ values referenced in Table 1 , a distance modulus of 24.47 (McConnachie et al. 2005) and the extinction parameter $R_{V}=3.1$. Figure 5 confirms that the M31 GCs generally show the same trend as MW GCs (data taken from Dubath et al. 1997; Harris 1996, 2010 revision), and that the M31 sample

\footnotetext{
4 http://www.ucolick.org/ xavier/IDL/index.html
} 
Table 1

Observations and Cluster Properties

\begin{tabular}{|c|c|c|c|c|c|c|c|c|}
\hline Name & $\begin{array}{c}\text { R.A. } \\
\text { (J2000) }\end{array}$ & $\begin{array}{c}\text { Decl. } \\
(\mathrm{J} 2000)\end{array}$ & $V$ & $E(B-V)$ & $\begin{array}{l}R_{\mathrm{M} 31} \\
(\mathrm{kpc})\end{array}$ & Date & $\begin{array}{l}T_{\exp } \\
(\mathrm{hr})\end{array}$ & $\begin{array}{c}\mathrm{S} / \mathrm{N}\left(\mathrm{pixel}^{-1}\right) \\
(6040 \AA)\end{array}$ \\
\hline B006-G058 & $00: 40: 26.5$ & $+41: 27: 26.4$ & 15.46 & 0.17 & 6.39 & 2008 Sep & 3.0 & 91 \\
\hline B012-G064 & $00: 40: 32.5$ & $+41: 21: 44.2$ & 15.04 & 0.17 & 5.74 & 2008 Sep & 2.1 & 86 \\
\hline B029-G090 & $00: 41: 17.8$ & $+41: 00: 22.8$ & 16.58 & 0.27 & 6.78 & 2011 Sep & 5.0 & 62 \\
\hline B034-G096 & $00: 41: 28.1$ & $+40: 53: 49.6$ & 15.47 & 0.16 & 6.02 & 2010 Oct & 4.0 & 100 \\
\hline B048-G110 & $00: 41: 45.5$ & $+41: 13: 30.7$ & 16.51 & 0.36 & 2.59 & 2011 Sep & 4.0 & 76 \\
\hline B088-G150 & $00: 42: 21.1$ & $+41: 32: 14.3$ & 15.00 & $0.46^{\mathrm{a}}$ & 3.80 & $\begin{array}{l}2009 \text { Sep } \\
2011 \text { Sep }\end{array}$ & $\begin{array}{l}1.2 \\
2.0\end{array}$ & $85^{\mathrm{e}}$ \\
\hline B110-G172 & $00: 42: 33.1$ & $+41: 03: 28.4$ & 15.28 & 0.12 & 2.93 & 2010 Oct & 3.0 & 112 \\
\hline B163-G217 & $00: 43: 17.0$ & $+41: 27: 44.9$ & 15.04 & 0.21 & 3.00 & 2012 Sep & 2.7 & 114 \\
\hline B171-G222 & $00: 43: 25.0$ & $+41: 15: 37.1$ & 15.28 & 0.19 & 1.77 & 2012 Sep & 3.0 & 91 \\
\hline B182-G233 & $00: 43: 36.7$ & $+41: 08: 12.2$ & 15.43 & 0.33 & 2.88 & 2010 Oct & 4.0 & 105 \\
\hline B193-G244 & $00: 43: 45.5$ & $+41: 36: 57.5$ & 15.33 & 0.23 & 5.41 & 2010 Oct & 1.1 & 56 \\
\hline B225-G280 & $00: 44: 29.8$ & $+41: 21: 36.6$ & 14.15 & 0.12 & 4.68 & 2008 Sep & 1.0 & 106 \\
\hline B232-G286 & $00: 44: 40.5$ & $+41: 15: 01.4$ & 15.65 & 0.21 & 4.96 & 2008 Sep & 3.0 & 87 \\
\hline B235-G297 & $00: 44: 57.9$ & $+41: 29: 23.7$ & 16.27 & 0.14 & 6.46 & 2011 Sep & 4.0 & 72 \\
\hline B240-G302 & $00: 45: 25.2$ & $+41: 06: 23.8$ & 15.18 & 0.13 & 7.22 & 2008 Sep & 3.0 & 98 \\
\hline B311-G033 & $00: 39: 33.8$ & $+40: 31: 14.4$ & 15.45 & 0.36 & 13.06 & 2010 Oct & 4.0 & 84 \\
\hline B312-G035 & $00: 39: 40.1$ & $+40: 57: 02.3$ & 15.52 & 0.23 & 9.02 & 2008 Sep & 3.0 & 80 \\
\hline B383-G318 & $00: 46: 12.0$ & $+41: 19: 43.2$ & 15.30 & 0.20 & 8.92 & 2009 Sep & 2.0 & 69 \\
\hline B384-G319 & $00: 46: 21.9$ & $+40: 17: 00.0$ & 15.75 & 0.10 & 16.42 & 2008 Sep & 3.5 & 91 \\
\hline B403-G348 & $00: 49: 17.0$ & $+41: 35: 08.2$ & 16.22 & 0.26 & 17.34 & 2012 Sep & 5.0 & 77 \\
\hline B457-G097 & $00: 41: 29.0$ & $+42: 18: 37.7$ & 16.91 & 0.13 & 14.58 & 2012 Sep & 6.0 & 54 \\
\hline B514-MCGC4 & $00: 31: 09.8$ & $+37: 53: 59.6$ & 15.76 & $0.09^{b}$ & 55.30 & 2008 Sep & 3.5 & 72 \\
\hline G327-MVI & $00: 46: 49.6$ & $+42: 44: 44.6$ & 15.90 & $0.18^{\mathrm{a}}$ & 22.70 & 2009 Sep & 3.0 & 68 \\
\hline G002 & $00: 33: 33.8$ & $+39: 31: 18.5$ & 15.93 & $0.08^{c}$ & 33.62 & 2008 Sep & 3.6 & 85 \\
\hline MCGC5-H10 & $00: 35: 59.8$ & $+35: 41: 03.9$ & 16.09 & $0.05^{b}$ & 78.68 & 2008 Sep & 4.3 & 77 \\
\hline MGC1 & $00: 50: 42.5$ & $+32: 54: 58.7$ & 15.50 & $0.17^{\mathrm{d}}$ & 117.05 & 2008 Sep & 3.2 & 67 \\
\hline \multicolumn{9}{|c|}{ Clusters analyzed in C09 } \\
\hline B045-G108 & $00: 41: 43.1$ & $+41: 34: 20.0$ & 15.83 & 0.18 & 4.90 & 2006 Sep & 4.5 & 100 \\
\hline B358-G219 & $00: 43: 17.9$ & $+39: 49: 13.2$ & 15.12 & $0.06^{\mathrm{a}}$ & 19.86 & 2006 Sep & 2.9 & 110 \\
\hline B381-G315 & $00: 46: 06.6$ & $+41: 20: 58.9$ & 15.76 & 0.24 & 8.72 & 2006 Sep & 4.0 & 100 \\
\hline B386-G322 & $00: 46: 27.0$ & $+42: 01: 52.8$ & 15.64 & 0.18 & 14.08 & 2006 Sep & 3.5 & 90 \\
\hline B405-G351 & $00: 49: 39.8$ & $+41: 35: 29.7$ & 15.20 & 0.18 & 18.28 & 2006 Sep & 3.0 & 100 \\
\hline
\end{tabular}

Notes. Cluster identifications, positions, $V$ magnitudes, and projected galactocentric radii from M31 are taken from the Revised Bologna Catalog (Galleti et al. 2004). Reddening values are taken from Caldwell et al. (2011), with the exceptions of ${ }^{\mathrm{a}}$ : Fan et al. (2008), ${ }^{\mathrm{b}}$ : Mackey et al. (2007), ${ }^{\mathrm{c}}$ : Barmby et al. (2007),

d: Mackey et al. (2010a), ${ }^{\text {e: }} \mathrm{S} / \mathrm{N}$ of combined 2009 and 2011 spectrum.

is mainly found in the more luminous range because of our selection criteria. Figure 5 also shows on the inset axis the corresponding effective spectral resolution of the GCs due to the velocity dispersion broadening, which can be found on the inset axis. This shows the large range in effective resolution, and that nearly all of the GC spectra are completely resolved with an instrumental setup of $R=24,000$.

\section{ABUNDANCE AND AGE ANALYSIS}

Our method for obtaining detailed abundances from IL GC spectra was presented for a pilot sample of M31 GCs in C09. In that paper, we performed an IL EW analysis using our routine ILABUNDS (MB08). In this work, we initially repeat that analysis for Fe I lines, which is reviewed in Section 3.1, and then refine that analysis as needed with an additional IL spectrum synthesis analysis, as described in Section 3.2 below.

\subsection{IL EW Analysis}

As described in C09, absorption line EWs are measured with the semi-automated program GETJOB (McWilliam et al. 1995b). The line lists used in our analysis are taken from references compiled in McWilliam \& Rich (1994), McWilliam et al. (1995a), McWilliam (1998), MB08 and Johnson et al. (2006).

ILABUNDS utilizes the 2010 version of MOOG (Sneden 1973) to calculate flux-weighted IL EWs to match to the observed EWs. In order to calculate IL EWs, we construct synthetic color-magnitude diagrams (CMDs) from Teramo isochrones (Pietrinferni et al. 2004, 2006; Cordier et al. 2007). We use canonical isochrones with an extended asymptotic giant branch (AGB), $\alpha$-enhanced low-temperature opacities calculated according to Ferguson et al. (2005), and a mass loss parameter of $\eta=0.2$. As in all of our analyses, we apply an initial mass function of the form in Kroupa (2002). The synthetic CMDs are divided into $\sim 25$ boxes of stars with similar properties, with each containing $\sim 4 \%$ of the total $V$-band flux. The atmospheres of the average stellar types are interpolated from the one-dimensional, plane parallel ODFNEW and AODFNEW model grids of $\mathrm{Kurucz}^{5}$ (e.g., Castelli \& Kurucz 2004). All abundances in both the EW and line synthesis analysis are calculated under the assumption of local thermodynamic equilibrium (LTE). All abundance ratios

\footnotetext{
5 The models are available from R. L. Kurucz's Web site at http://kurucz.harvard.edu/grids.html.
} 


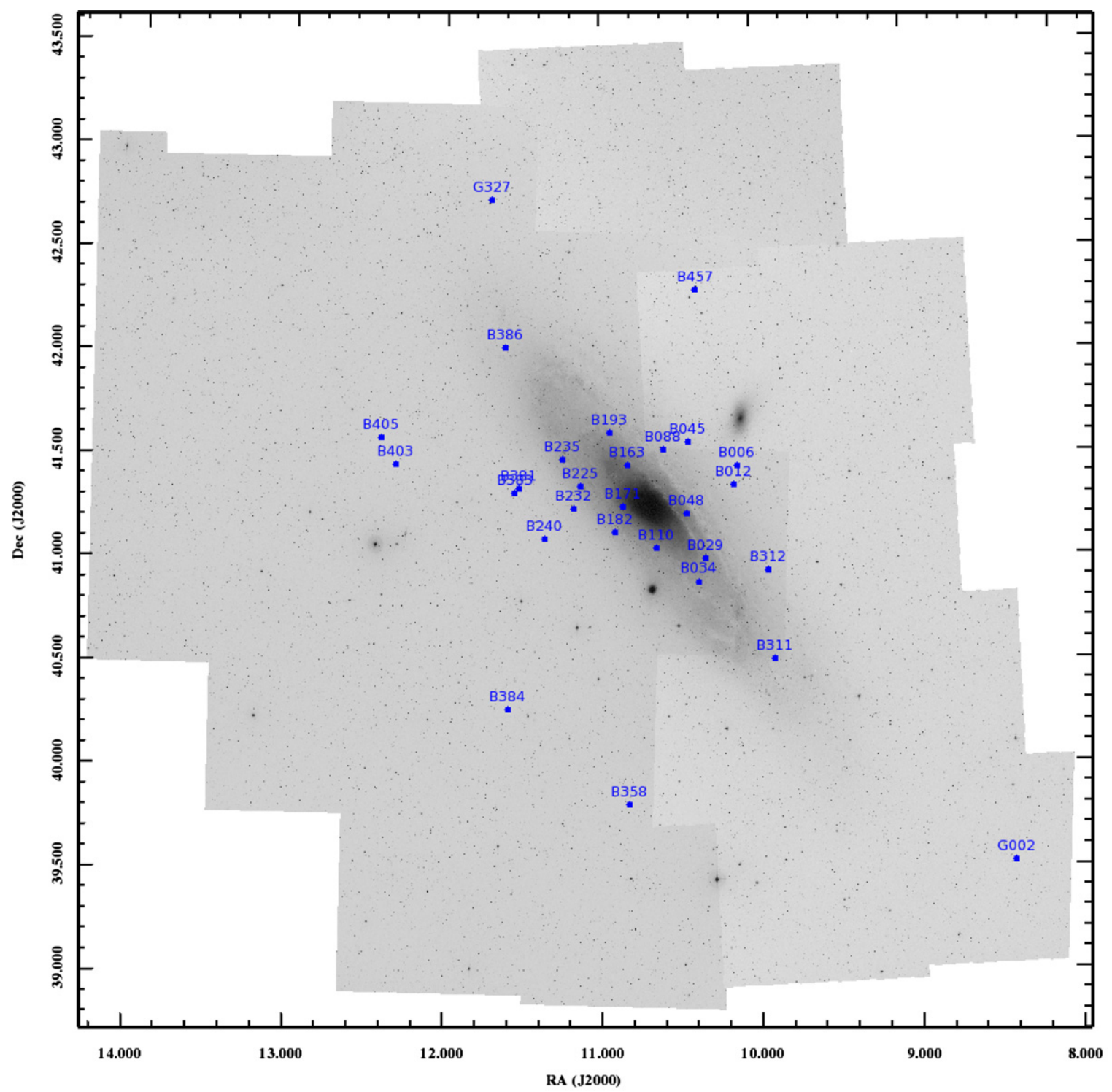

Figure 1. M31 GC targets superimposed over a composite STScI Digitized Sky Survey image of M31 and the surrounding field. Note that B514, MCGC5, and MGC1 are beyond the edges of the field and have galactocentric radii of 55, 79, and $117 \mathrm{kpc}$, respectively. For comparison, the next most distant GC from the center of M31 in our sample is G002, which has a galactocentric radius of $34 \mathrm{kpc}$.

(A color version of this figure is available in the online journal.)

relative to solar are calculated with the solar abundance values of Asplund et al. (2009).

The age and $[\mathrm{Fe} / \mathrm{H}]$ solutions for each cluster are identified as the range in synthetic $\mathrm{CMD}$ ages and $[\mathrm{Fe} / \mathrm{H}]$ that produce the most self-consistent results using the 10-80 individual $\mathrm{Fe}_{\mathrm{I}}$ lines measured in each cluster. The best solutions have the smallest line-to-line statistical error $\left(\sigma_{\mathrm{N}}\right)$, and minimal dependence of $\mathrm{Fe}$ I abundance with line excitation potential (EP), wavelength, and EW. The line-to-line scatter also includes systematic uncertainties between the lines themselves. For each cluster, there is a range in CMD ages that produce similarly self-consistent solutions. For older clusters, this range is typically $10-15 \mathrm{Gyr}$ and leads to a systematic uncertainty in $[\mathrm{Fe} / \mathrm{H}]$ of $\lesssim 0.05 \mathrm{dex}$, which we denote $\sigma_{\mathrm{Age}}$. For younger clusters, the preferred age range may be smaller, but the $\sigma_{\text {Age }}$ can be larger $(\sim 0.1 \mathrm{dex})$ due to the more rapidly changing stellar populations at younger ages. For the total uncertainty in $[\mathrm{Fe} / \mathrm{H}]$ for each cluster, we add the statistical error in the mean abundance $\left(\sigma_{\mathrm{N}} / \sqrt{N-1}\right)$ (which also includes systematic errors between lines) and the systematic age uncertainty $\sigma_{\mathrm{Age}}$ in quadrature.

\subsection{Refined Fe Line Synthesis Analysis}

Since the publication of our pilot study in C09, we have further refined our abundance analysis techniques using the IL spectral synthesis component of ILABUNDS (C11). In C11, we implemented a $\chi^{2}$ minimization scheme with the IL spectral synthesis in order to recover more elemental abundances from lower $\mathrm{S} / \mathrm{N}$ data $(\mathrm{S} / \mathrm{N} \sim 40)$. This type of analysis can also improve the precision of the measurements in cases where line blending is significant because unlike EW analysis of single features, the synthesis includes the contributions from all the nearby lines in a specified region. It also allows for more accurately establishing the "pseudo" continuum around the lines of interest (see Sakari et al. 2013 for a more detailed discussion of continuum in IL spectra). In terms of the analysis of GC IL spectra, line blending is most significant when the overall 
Table 2

Velocity Dispersions and Radial Velocities

\begin{tabular}{|c|c|c|c|c|c|c|c|c|c|}
\hline \multirow[b]{2}{*}{ Name } & \multicolumn{4}{|c|}{ This Work } & \multicolumn{5}{|c|}{ Literature } \\
\hline & $\begin{array}{c}v_{\sigma} \\
\left(\mathrm{km} \mathrm{s}^{-1}\right)\end{array}$ & $\begin{array}{c}\text { Error } \\
\left(\mathrm{km} \mathrm{s}^{-1}\right)\end{array}$ & $\begin{array}{c}v_{r} \\
\left(\mathrm{~km} \mathrm{~s}^{-1}\right)\end{array}$ & $\begin{array}{c}\text { Error } \\
\left(\mathrm{km} \mathrm{s}^{-1}\right)\end{array}$ & $\begin{array}{c}v_{\sigma, \mathrm{Lit}} \\
\left(\mathrm{km} \mathrm{s}^{-1}\right)\end{array}$ & $\begin{array}{c}\text { Error } \\
\left(\mathrm{km} \mathrm{s}^{-1}\right)\end{array}$ & $\begin{array}{c}v_{r, \mathrm{Lit}} \\
\left(\mathrm{km} \mathrm{s}^{-1}\right)\end{array}$ & $\begin{array}{c}\text { Error } \\
\left(\mathrm{km} \mathrm{s}^{-1}\right)\end{array}$ & Ref. \\
\hline B006-G058 & 11.93 & 0.45 & -238.1 & 0.2 & 11.9 & 0.7 & -236.5 & 0.6 & $1,3,5$ \\
\hline B012-G064 & 19.50 & 0.76 & -359.4 & 0.1 & 17.8 & 2.2 & -360.7 & 0.6 & 1,5 \\
\hline B029-G090 & 6.51 & 0.74 & -520.1 & 0.3 & 6.8 & 0.6 & -505.2 & 0.6 & 1 \\
\hline B034-G096 & 12.38 & 0.41 & -555.5 & 0.4 & $\ldots$ & $\ldots$ & -539 & 6 & 2 \\
\hline B048-G110 & 7.42 & 0.73 & -241.7 & 0.1 & 7.1 & 0.5 & -228.1 & 0.5 & 1 \\
\hline B088-G150 & 14.25 & 0.90 & -491.9 & 0.2 & 16.5 & 1.0 & -489.4 & 0.6 & 1 \\
\hline B110-G172 & 18.20 & 0.54 & -238.4 & 0.6 & 19.6 & 1.1 & -237.0 & 0.5 & 1 \\
\hline B163-G217 & 17.41 & 0.83 & -174.8 & 0.1 & 18.8 & 1.0 & -163.5 & 0.5 & 1 \\
\hline B171-G222 & 15.04 & 0.81 & -287.6 & 0.1 & 15.6 & 0.9 & -267.5 & 0.5 & 1 \\
\hline B182-G233 & 19.29 & 0.57 & -361.7 & 0.5 & 18.4 & 1.0 & -356.6 & 0.5 & 1 \\
\hline B193-G244 & 15.79 & 0.37 & -59.4 & 0.5 & 14.7 & 2.1 & -62.1 & 0.5 & 1,5 \\
\hline B225-G280 & 25.73 & 1.14 & -154.2 & 0.2 & 27.2 & 1.6 & -163.7 & 1.6 & $1,3,5$ \\
\hline B232-G286 & 14.24 & 0.72 & -188.3 & 0.3 & 13.3 & 0.8 & -191.7 & 0.6 & 1 \\
\hline B235-G297 & 8.20 & 0.59 & -106.7 & 0.3 & 8.2 & 0.5 & -92.4 & 0.4 & 1 \\
\hline B240-G302 & 12.23 & 0.48 & -53.1 & 0.2 & 12.4 & 0.6 & -55.8 & 2.0 & 1,5 \\
\hline B311-G033 & 14.01 & 0.50 & -514.0 & 0.2 & $\ldots$ & $\ldots$ & -469 & 10 & 2 \\
\hline B312-G035 & 12.91 & 1.24 & -172.6 & 0.3 & $\ldots$ & $\ldots$ & -174 & 11 & 2 \\
\hline B383-G318 & 11.13 & 0.40 & -231.1 & 0.3 & $\ldots$ & $\ldots$ & -253 & 9 & 2 \\
\hline B384-G319 & 9.00 & 0.56 & -359.4 & 0.3 & 10.3 & 0.4 & -363.8 & 0.3 & 2,5 \\
\hline B403-G348 & 9.70 & 0.39 & -366.0 & 0.3 & $\ldots$ & $\ldots$ & -358 & 48 & 2 \\
\hline B457-G097 & 4.73 & 0.80 & -72.1 & 0.3 & $\ldots$ & $\ldots$ & -63 & 15 & 2 \\
\hline B514-MCGC4 & 8.49 & 0.55 & -474.7 & 0.1 & $\ldots$ & $\ldots$ & -458 & 23 & 2 \\
\hline G327-MVI & 8.43 & 0.68 & -270.3 & 0.2 & $\ldots$ & $\ldots$ & -251 & 11 & 2 \\
\hline G002 & 10.12 & 0.52 & -349.3 & 0.2 & 9.7 & 0.3 & -313 & 17 & 5 \\
\hline MCGC5-H10 & 7.12 & 0.80 & -354.6 & 0.1 & 7.2 & 0.4 & -358.3 & 1.9 & 4 \\
\hline MGC1 & 8.29 & 0.72 & -354.6 & 0.1 & $\ldots$ & $\ldots$ & -355 & 2 & 2 \\
\hline
\end{tabular}

References. 1. Strader et al. 2011. 2. Galleti et al. 2004. 3. Dubath \& Grillmair 1997. 4. Alves-Brito et al. 2009. 5. Djorgovski et al. 1997. Where two references are listed, we have calculated the average of the two results and an error equal to the standard deviation in the mean.

metallicity is high, and/or when the cluster's velocity dispersion is large. Therefore, it is especially important to evaluate the impact of line blending on our analysis when we apply our technique to clusters in galaxies whose GC systems are thought to reach higher overall metallicities than the MW's GC system. In addition, this is important for analyzing GCs in more distant galaxies where we are observationally limited to probing the brightest, most massive, portion of the GC luminosity function. In this case, the GCs we can observe will likely have larger velocity dispersions than the typical GCs that we can observe in the Local Group (see Colucci et al. 2013b for massive GCs in NGC 5128).

Our refined Fe line synthesis analysis first consists of an automated procedure to synthesize a region $\pm 10 \AA$ around each Fe I feature in the preferred line list used for our EW analysis, which was described above. As in C11, the wavelengths, $g f$ values, and other atomic parameters of the neighboring features around the preferred $\mathrm{Fe}$ I lines are drawn from the larger Kurucz database. ${ }^{6}$ Other than the line list, to calculate the IL spectra we only need input a synthetic CMD, which has its own associated $[\mathrm{Fe} / \mathrm{H}]$ that is used as a starting abundance. The procedure then synthesizes IL spectra in each region with abundances that vary from $\pm 0.5 \mathrm{dex}$ from the initial $[\mathrm{Fe} / \mathrm{H}]$, in steps of $0.1 \mathrm{dex}$. We have found that for the typical $\mathrm{S} / \mathrm{Ns}$ we obtain, 0.1 dex increments in $[\mathrm{Fe} / \mathrm{H}]$ are sufficient for discriminating meaningful abundance differences in Fe I lines with different atomic parameters across the full wavelength range. In principle, however, this choice is arbitrary and a smaller increment could be used for

\footnotetext{
6 http://kurucz.harvard.edu/linelists.html
}

exceptional quality data. We note that this step size is a negligible source of uncertainty compared to the systematics, which are demonstrated by the line-to-line scatter when multiple lines are available and the uncertainty due to the unknown age of the $\mathrm{GC}$, which is represented in our analysis by the range in CMD ages we use. The total uncertainty for any abundance measured from multiple lines is estimated from the line-to-line scatter $\left(\sigma_{\mathrm{N}}\right)$, which will include systematic uncertainties between the lines themselves. These systematics are more difficult to quantify (oscillator strengths, incomplete line lists, effect of stellar population mismatch, etc.) than the statistical errors associated with measuring the EW, which are much smaller.

In order to perform a meaningful $\chi^{2}$ minimization, we must appropriately compare the observed spectra with the synthesized spectra, as described in C11. As a starting point, we automatically normalize the data to an average of the top $\sim 100$ maximum values of the flux across the pixels in the $20 \AA$ region of each synthesis. We bias the normalization to the highest flux values, with a reasonable allocation for the noise level, in order to account for absorption lines preferentially lowering the pseudo-continuum. The $\chi^{2}$ minimization can then be performed in a region that is approximately $\pm 0.25 \AA$ around the Fe I line. As pointed out in $\mathrm{C} 11$, in practice one must review the data synthesis comparison around each Fe I line by eye in order to obtain the most accurate measurements. First, it is particularly important to evaluate the normalization over a broad region, because subtle differences in matching the pseudo-continuum in high metallicity or highly broadened cluster spectra can greatly impact the results. Note that in our analysis, we have chosen to isolate the $\mathrm{Fe}$ I lines of interest rather than performing a $\chi^{2}$ 


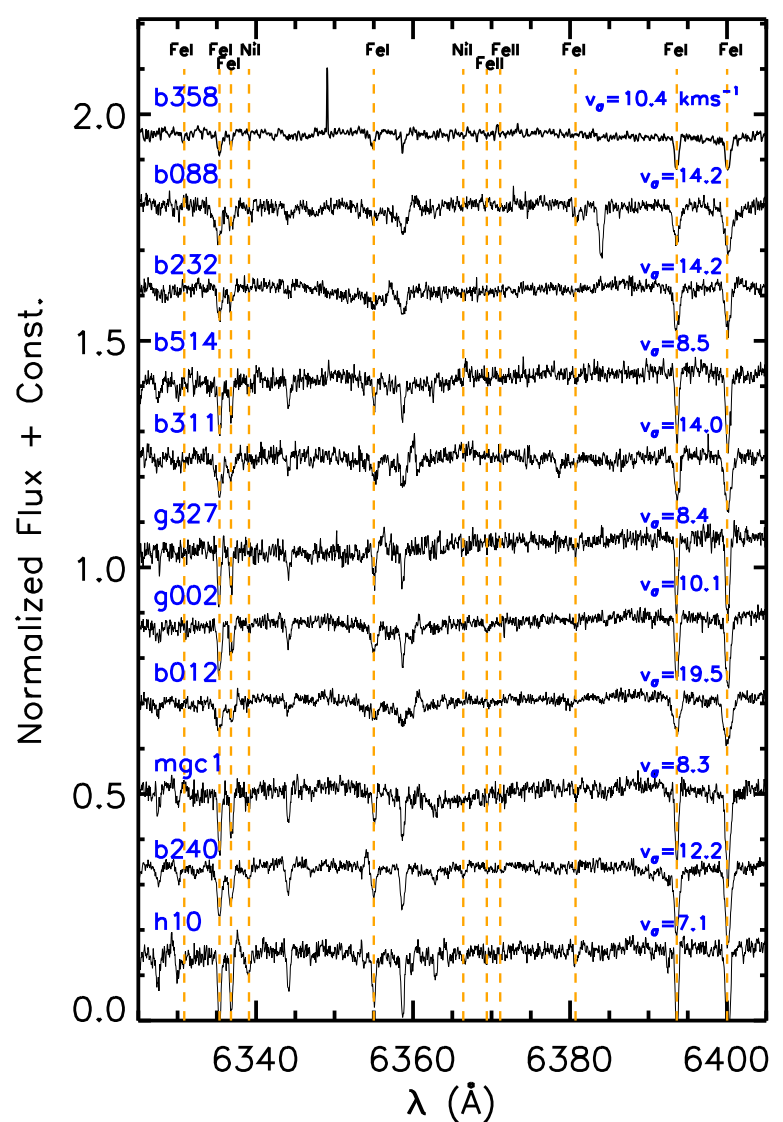

Figure 2. Portion of the spectrum of each of the 11 most metal-poor GCs in our sample. The spectra are normalized to 1.0 , with a base at 0.0 , and a constant offset has been applied to each for visualization. Metallicity increases from top to bottom. The cluster names are shown, as well as the velocity dispersions we measure in Section 2.1. Dashed lines correspond to Fe I, Fe II, and Ni I transitions, as noted.

(A color version of this figure is available in the online journal.)

minimization over the full $20 \AA$ region. We choose to do this because we are interested in specific Fe I transitions with the most accurate $g f$ values, and we want to minimize uncertainties introduced by an (unavoidably) more uncertain extended line list in the region. By reviewing each line, we can also eliminate Fe lines that are too badly blended to provide a meaningful measurement, those that may have strong non-LTE effects, lines that are in areas of particularly bad local noise, those that are coincident with sky absorption lines, etc.

Once the cleanest set of $\mathrm{Fe}$ I lines is established in this way, the rest of the $[\mathrm{Fe} / \mathrm{H}]$ and age analysis proceeds automatically in the same way as in the EW analysis. We use the mean abundance derived from the set of Fe I lines to iteratively solve for a selfconsistent CMD at each age, where the input CMD abundance is equal to the final derived abundance for all lines. This results in one self consistent synthetic CMD of a given $[\mathrm{Fe} / \mathrm{H}]$ at each age. Then, because we are still isolating individual $\mathrm{Fe}$ lines, we use the same diagnostics to constrain the most appropriate age for each GC; namely the self-consistency of the abundance from individual $\mathrm{Fe}$ lines as a function of wavelength, EP, and EW. The one difference is that in this case we must calculate a "pseudo-EW" for each line, which is essentially the EW we would have observed in the absence of line blending. In order to do this, we re-synthesize IL spectra with the final inferred $[\mathrm{Fe} / \mathrm{H}]$ using only the single Fe I transitions in our line list, and then calculate the inferred, pseudo-EWs for each Fe line.

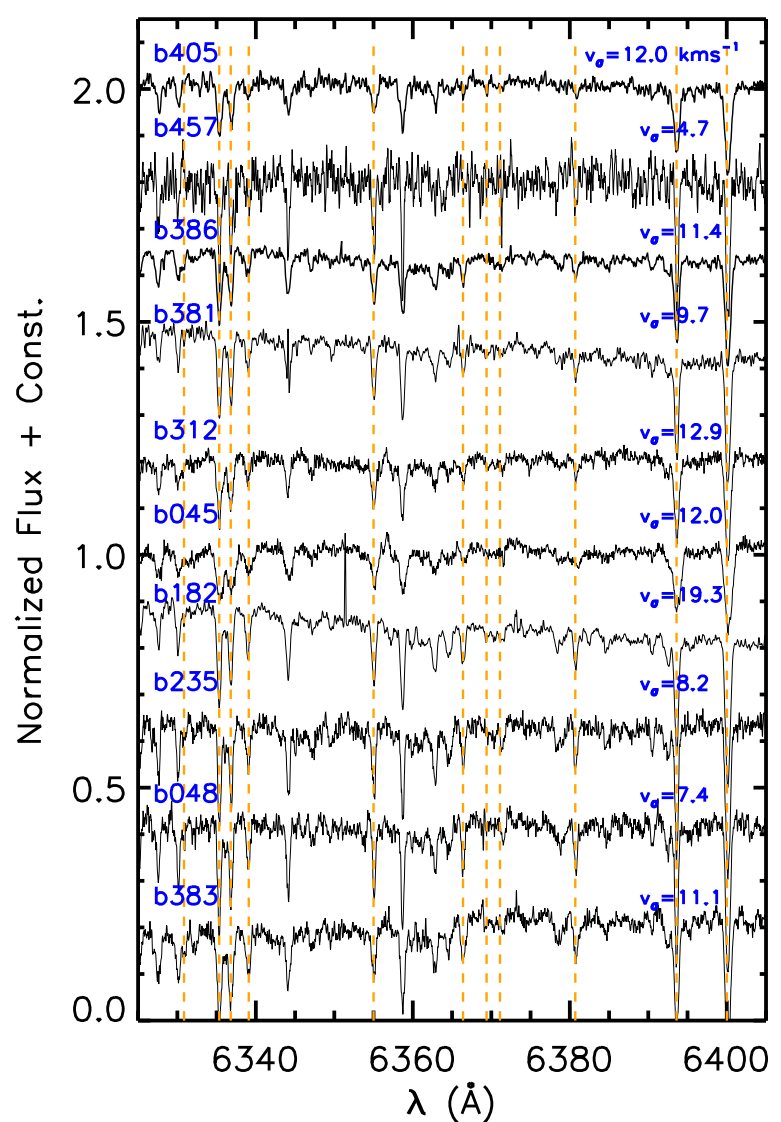

Figure 3. Same as Figure 2 for the 10 intermediate metallicity GCs in our sample.

(A color version of this figure is available in the online journal.)

This refined Fe line synthesis analysis is much more time and user intensive than the semi-automated EW analysis. It is also more user intensive than other techniques that rely on automated full spectrum fitting. However, GC IL spectra are fundamentally complex, and there are more measurement subtleties than in analysis of individual stars. These uncertainties are only compounded when full spectrum fitting over regions with poorly calibrated line lists, large non-LTE effects, or strong lines that are not on the linear region of the curve of growth. The refined $\mathrm{Fe}$ line synthesis analysis outlined here makes it possible to recover precise, reliable abundance information from GC IL spectra that have $\mathrm{S} / \mathrm{N}$ too low for standard $\mathrm{EW}$ analysis or are significantly affected by line blending due to high overall metallicity and/or large velocity broadening.

Due to the intensiveness of this technique for determining the overall $[\mathrm{Fe} / \mathrm{H}]$ of a $\mathrm{GC}$, we have performed tests to establish the situations where EW analysis is not sufficient for measuring accurate abundances and ages. We highlight some examples using the M31 sample in the next section.

\subsubsection{EW versus Synthesis Tests}

We initially performed an EW analysis for all the GCs in our sample. Next we performed a refined Fe line synthesis analysis for a subset of the GCs with large $v_{\sigma}$ and/or high $[\mathrm{Fe} / \mathrm{H}]$. To begin, the subset included all of the GCs with velocity dispersions greater than $15 \mathrm{~km} \mathrm{~s}^{-1}$, as well as all of the $\mathrm{GCs}$ with $[\mathrm{Fe} / \mathrm{H}]>-0.5$. Note that $\mathrm{B} 225$, which has the largest velocity dispersion by far, is not included in this subset because we were not able to perform an EW analysis with GETJOB. 


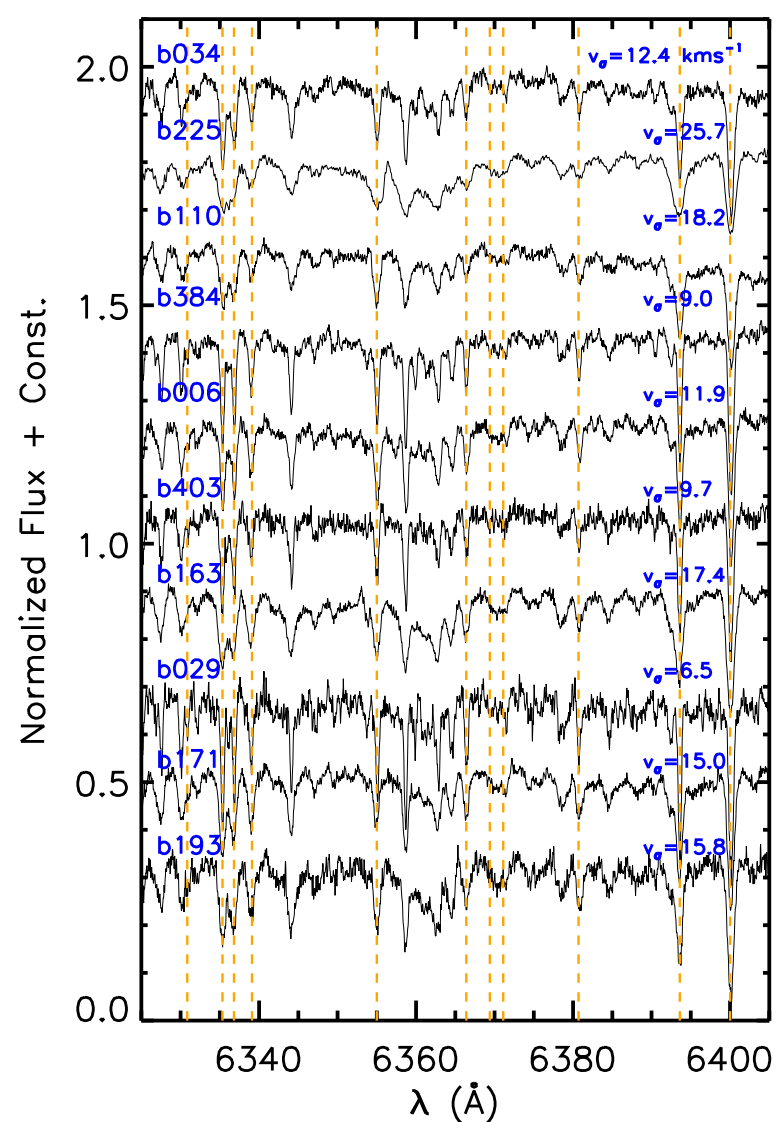

Figure 4. Same as Figure 2 for the 11 most metal-rich GCs in our sample. (A color version of this figure is available in the online journal.)

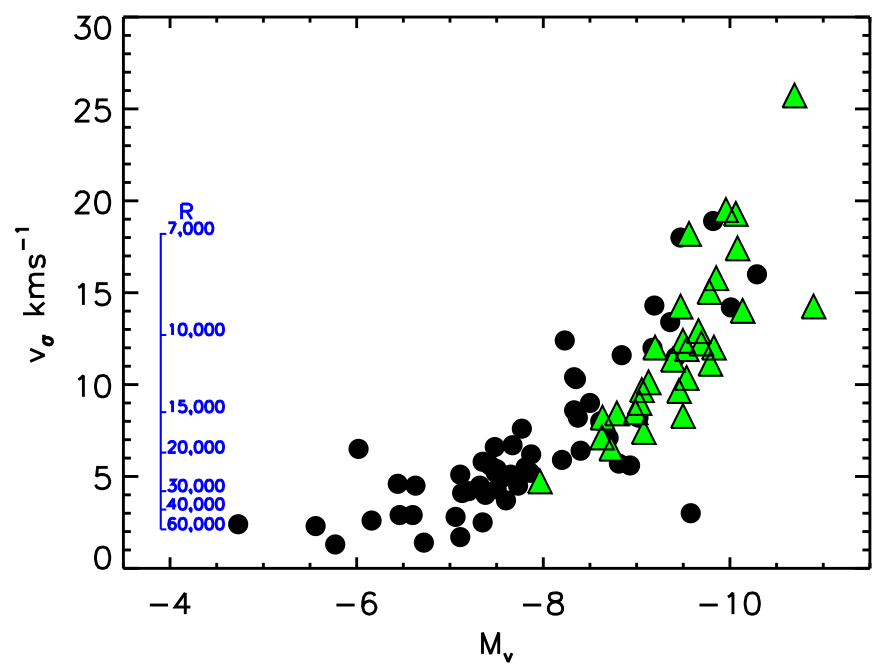

Figure 5. Green triangles show our velocity dispersion measurements for the M31 sample as a function of reddening corrected absolute $V$ magnitude. Reddening and magnitudes are listed in Table 1, and we assume a distance modulus of 24.47 (McConnachie et al. 2005) and extinction parameter of $R_{v}=$ 3.1. For comparison, a subset of the MW GC system is shown in black; data are taken from Dubath et al. (1997) and the 2010 revision of the Harris (1996) catalog. The inset axis shows the effective spectral resolution corresponding to the velocity dispersion broadening.

(A color version of this figure is available in the online journal.)

Some of the GCs presented here satisfy both of the criteria. In addition, we performed the synthesis for a subset of GCs that sample the rest of the range of velocity dispersion and $[\mathrm{Fe} / \mathrm{H}]$ in order to establish the parameter space where EW analysis
Table 3

EW versus Synthesis Analysis

\begin{tabular}{lcccccc}
\hline \hline \multirow{2}{*}{ Name } & \multicolumn{2}{c}{ EW } & & \multicolumn{2}{c}{ Synthesis } & \multirow{2}{*}{$v_{\sigma}$} \\
\cline { 2 - 3 } & {$[\mathrm{Fe} / \mathrm{H}]$} & $\sigma$ & & {$[\mathrm{Fe} / \mathrm{H}]$} & $\sigma$ & \\
\hline B029-G090 & -0.39 & 0.19 & & -0.43 & 0.11 & 6.5 \\
B514-MCGC4 & -1.74 & 0.17 & & -1.81 & 0.13 & 8.5 \\
B384-G319 & -0.71 & 0.15 & & -0.63 & 0.14 & 9.0 \\
B403-G348 & -0.68 & 0.18 & & -0.80 & 0.16 & 9.7 \\
B383-G318 & -0.78 & 0.16 & & -0.80 & 0.14 & 11.1 \\
B006-G058 & -0.58 & 0.22 & & -0.70 & 0.15 & 11.9 \\
B034-G096 & -0.65 & 0.29 & -0.75 & 0.14 & 12.4 \\
B311-G033 & -1.71 & 0.19 & & -1.75 & 0.12 & 14.0 \\
B171-G222 & -0.46 & 0.24 & & -0.45 & 0.19 & 15.0 \\
B193-G244 & -0.30 & 0.40 & -0.16 & 0.12 & 15.8 \\
B163-G217 & -0.26 & 0.34 & -0.49 & 0.18 & 17.4 \\
B110-G172 & -0.74 & 0.15 & -0.66 & 0.10 & 18.2 \\
B182-G233 & -1.28 & 0.36 & -1.04 & 0.18 & 19.3 \\
B012-G064 & -1.61 & 0.21 & -1.71 & 0.20 & 19.5 \\
\hline
\end{tabular}

produces results as accurate as the synthesis. The full test subset of GCs and the results from both analyses are listed in Table 3 .

In Figure 6, we show the general trends in the comparison between EW and synthesis analysis. We have quantified the differences in two ways; first in terms of the difference in the final derived $[\mathrm{Fe} / \mathrm{H}]$, and second in terms of the reduction of the statistical error $\left(\sigma_{\mathrm{N}}\right)$, i.e., the scatter in abundance between individual Fe I lines. We show both of these quantities as a function of $[\mathrm{Fe} / \mathrm{H}]$ and $v_{\sigma}$ in Figures $6(\mathrm{a})$ and (c) and Figures 6(b) and (d), respectively. For the $[\mathrm{Fe} / \mathrm{H}]$ comparison, we also show the $1 \sigma$ error bars $\left(\sigma_{\mathrm{N}}\right)$ as a guide for evaluating consistent results between analyses.

Inspection of Figure 6 seems to imply that large velocity dispersions have a bigger impact on the results than high $[\mathrm{Fe} / \mathrm{H}]$. In general, the naive expectation is true in that line synthesis analysis results in abundances that are slightly lower than EW analysis; however, this is not necessarily the case for solutions that had large statistical errors $\left(\sigma_{\mathrm{N}}\right)$ to begin with. As might be expected, the most discrepant cases occur for GCs that have both a large velocity dispersion and a high $[\mathrm{Fe} / \mathrm{H}]$. At worst, the $[\mathrm{Fe} / \mathrm{H}]$ differs by $\sim 0.25 \mathrm{dex}$, and the statistical error can be reduced by $\sim 0.25$ dex, which is a change of $70 \%$. Nonetheless, in most other cases the differences are less dramatic. Figure 6(a) shows that the $[\mathrm{Fe} / \mathrm{H}]$ results are consistent within $1 \sigma$ for all of the GCs that have velocity dispersions $<15 \mathrm{~km} \mathrm{~s}^{-1}$, and for the lowest metallicity clusters even the statistical errors $\left(\sigma_{\mathrm{N}}\right)$ are nearly identical in both cases, which means that EW abundance analysis is just as accurate as full synthesis analysis in this regime. Figure 6(b) more clearly shows that inconsistent $[\mathrm{Fe} / \mathrm{H}]$ are not seen until GC velocity dispersions are $>15 \mathrm{~km} \mathrm{~s}^{-1}$. Figure $6(\mathrm{~d})$ shows that the statistical errors can be significantly improved for GCs with velocity dispersions as low as $12 \mathrm{~km} \mathrm{~s}^{-1}$, even though the final $[\mathrm{Fe} / \mathrm{H}]$ for these GCs are not formally inconsistent between analyses. Our results suggest that $\mathrm{GCs}$ with $[\mathrm{Fe} / \mathrm{H}]$ at least as high as -0.4 can be accurately analyzed with EWs if the velocity dispersion is not a concern. The two GCs in our sample that have higher $[\mathrm{Fe} / \mathrm{H}]$ also have large velocity dispersions, but the most conservative conclusion is that the high $[\mathrm{Fe} / \mathrm{H}]$ is exacerbating the problem. Therefore, our conclusions from this analysis are that it is necessary to perform the more intensive line synthesis analysis for GCs with velocity dispersions $>15 \mathrm{~km} \mathrm{~s}^{-1}$, and those with $[\mathrm{Fe} / \mathrm{H}]>-0.3$. Finally, we note that none of the GCs in C09 

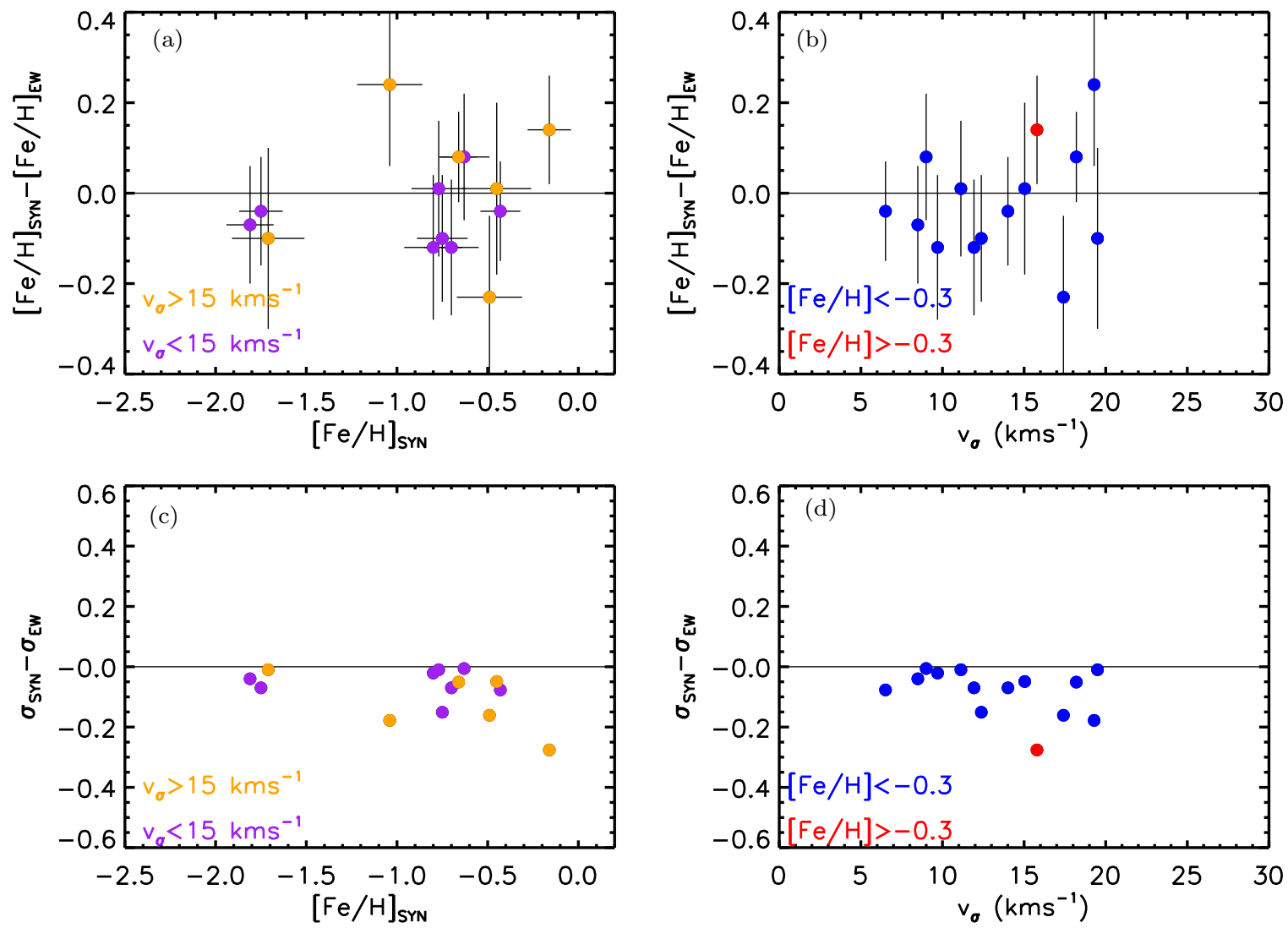

Figure 6. Results of our tests between EW and line synthesis analysis. (a) The difference in mean $[\mathrm{Fe} / \mathrm{H}]$ from EWs and synthesis as a function of the $[\mathrm{Fe} / \mathrm{H}]$ determined from line synthesis. GCs with velocity dispersions $>15 \mathrm{~km} \mathrm{~s}^{-1}$ are highlighted in orange, and those with $v_{\sigma}<15 \mathrm{~km} \mathrm{~s}$ are highlighted in purple. (b) The same as (a), instead as a function of velocity dispersion. Blue and red designate clusters with $[\mathrm{Fe} / \mathrm{H}]$ below and above -0.3 . (c) The difference in the statistical error of the mean $[\mathrm{Fe} / \mathrm{H}]$ between the two analyses as a function of $[\mathrm{Fe} / \mathrm{H}]$ determined from the line synthesis analysis. (d) The same as (c), only as a function of velocity dispersion.

(A color version of this figure is available in the online journal.)

meet these criteria, and thus the Fe results and conclusions from the EW analysis of $\mathrm{C} 09$ are unaffected.

The $v_{\sigma}$ and $[\mathrm{Fe} / \mathrm{H}]$ effects are straightforward to quantify, but we have also found other situations when a more intensive line synthesis is beneficial or even necessary. The first cases are for GCs where the data quality is low enough that the EW analysis doesn't converge to a $[\mathrm{Fe} / \mathrm{H}]$ solution with a reasonable statistical error. B457, which has a relatively low velocity dispersion of $4.7 \mathrm{~km} \mathrm{~s}^{-1}$, has the lowest $\mathrm{S} / \mathrm{N}$ in our sample $(\mathrm{S} / \mathrm{N}=54)$, and was difficult to analyze with EWs. For that reason, we use line synthesis for the final $\mathrm{Fe}$ I analysis. Note that the $\mathrm{S} / \mathrm{N}$ of $\mathrm{B} 193$ is similar $(\mathrm{S} / \mathrm{N}=56)$. For that cluster, synthesis analysis was used because it satisfies our velocity dispersion criterium $\left(v_{\sigma}=15.8\right)$. A second, perhaps more interesting, case occurred when the EW analysis suggested a GC had a young or intermediate age. Initially we found three GCs that appeared young when analyzed with EWs (B029, B034, B384). These three GCs are among the more metalrich GCs in our sample, so we were suspicious of how higher metallicity could subtly be influencing the results. We also note that the existence of intermediate age GCs in M31 is still heavily debated, so it is particularly important that we investigate any potential systematic effects that may cause us to measure young ages. Therefore, we performed the more indepth line synthesis analysis to be as confident as possible with the results. For B029, the line synthesis analysis didn't change the age determination, which is discussed in more detail in
Section 4.1.1. However, for B034 and B384, we found that the subtle measurement differences in the line synthesis analysis changed the age solutions so that they were more consistent with old ages. We suspect that the changes are due to the disproportionate influence of cool giants on strong, red Fe lines.

In Figures 7 and 8, we investigate the differences between the solutions in an attempt to determine what the cause may be. Here we use B384 as an example, but the same behaviors are seen in the solution for B034. In Figure 7, we show the change in the EW measured from GETJOB and that measured in the line synthesis analysis as a function of wavelength, and in Figure 8, we show the change in derived abundance as a function of wavelength. We have highlighted the strong lines, i.e., those Fe I lines that have EWs $>100 \mathrm{~m} \AA$. Unfortunately, we don't have any weak lines bluer than $6000 \AA$ to compare, but above $6000 \AA$, where we have both strong and weak lines, the strong lines always have large changes in EW between analyses. With these data, a general conclusion would be that the large changes in EW for strong red lines, which will also result in large changes in $[\mathrm{Fe} / \mathrm{H}]$, are primarily responsible for the differences in the final solutions between analyses. However, it is also possible that local issues (continuum, line blending) can be affecting this particular set of lines, so we will address this issue with a larger sample of metal-rich MW clusters in an upcoming paper (Colucci et al. 2014).

It is helpful to qualitatively observe how these subtle differences are manifested in the $\mathrm{Fe}$ abundance versus wavelength 
Table 4

M31 GC Fe I EWs

\begin{tabular}{|c|c|c|c|c|c|c|c|c|c|c|c|c|c|c|c|}
\hline $\begin{array}{l}\lambda \\
(\AA)\end{array}$ & $\begin{array}{c}\mathrm{EP} \\
(\mathrm{eV})\end{array}$ & $\log g f$ & $\begin{array}{l}\text { B006 } \\
(\mathrm{mA})\end{array}$ & $\begin{array}{l}\text { B048 } \\
(\mathrm{mA})\end{array}$ & $\begin{array}{l}\text { B088 } \\
(\mathrm{m} \AA)\end{array}$ & $\begin{array}{l}\mathrm{B} 232 \\
(\mathrm{~m} \AA)\end{array}$ & $\begin{array}{l}\mathrm{B} 235 \\
(\mathrm{~m} \AA)\end{array}$ & $\begin{array}{l}\mathrm{B} 240 \\
(\mathrm{~m} \AA)\end{array}$ & $\begin{array}{l}\text { B311 } \\
(\mathrm{mA})\end{array}$ & $\begin{array}{l}\text { B312 } \\
(\mathrm{mA})\end{array}$ & $\begin{array}{l}\text { B383 } \\
(\mathrm{m} \AA)\end{array}$ & $\begin{array}{l}\mathrm{B} 403 \\
(\mathrm{~m} \AA)\end{array}$ & $\begin{array}{l}\mathrm{B} 514 \\
(\mathrm{~m} \AA)\end{array}$ & $\begin{array}{l}\mathrm{G} 002 \\
(\mathrm{~m} \AA)\end{array}$ & $\begin{array}{l}\text { G327 } \\
(\mathrm{mA})\end{array}$ \\
\hline 6322.694 & 2.590 & -2.440 & 93.6 & 82.3 & $\ldots$ & $\ldots$ & 67.0 & $\ldots$ & $\ldots$ & 66.5 & 81.5 & 87.6 & $\ldots$ & $\ldots$ & $\ldots$ \\
\hline 6335.337 & 2.200 & -2.170 & 138.4 & 115.9 & $\ldots$ & $\ldots$ & 115.9 & 83.8 & $\ldots$ & $\ldots$ & 139.9 & 135.8 & $\ldots$ & $\ldots$ & $\ldots$ \\
\hline 6336.830 & 3.690 & -0.670 & 95.8 & 99.4 & $\ldots$ & $\ldots$ & 85.8 & $\ldots$ & $\ldots$ & 73.5 & 123.6 & 99.9 & 45.0 & $\ldots$ & 49.1 \\
\hline 6355.035 & 2.840 & -2.330 & $\ldots$ & 76.5 & $\ldots$ & $\ldots$ & $\ldots$ & $\ldots$ & $\ldots$ & 76.6 & 89.7 & 98.8 & $\ldots$ & .. & $\ldots$ \\
\hline 6380.750 & 4.190 & -1.370 & $\ldots$ & 51.6 & $\ldots$ & $\ldots$ & $\ldots$ & $\ldots$ & $\ldots$ & $\ldots$ & $\ldots$ & 57.6 & $\ldots$ & $\ldots$ & $\ldots$ \\
\hline 6393.612 & 2.430 & -1.500 & $\ldots$ & $\ldots$ & 85.0 & $\ldots$ & 138.8 & 114.3 & 76.0 & 130.2 & $\ldots$ & 149.8 & 80.0 & $\ldots$ & $\ldots$ \\
\hline 6411.658 & 3.650 & -0.650 & 140.9 & 97.0 & 45.4 & $\ldots$ & 120.2 & 80.5 & 64.0 & 88.7 & 113.7 & 124.4 & 74.8 & $\ldots$ & $\ldots$ \\
\hline 6421.360 & 2.280 & -1.980 & $\ldots$ & $\ldots$ & 83.8 & $\ldots$ & $\ldots$ & $\ldots$ & $\ldots$ & 118.1 & $\ldots$ & $\ldots$ & $\ldots$ & $\ldots$ & $\ldots$ \\
\hline 6475.632 & 2.560 & -2.930 & 88.5 & $\ldots$ & $\ldots$ & $\ldots$ & 66.9 & 32.4 & $\ldots$ & $\ldots$ & 58.5 & 82.6 & $\ldots$ & $\ldots$ & $\ldots$ \\
\hline 6481.878 & 2.280 & -2.980 & 94.2 & $\ldots$ & $\ldots$ & $\ldots$ & 94.0 & 45.3 & $\ldots$ & 74.8 & 83.2 & $\ldots$ & $\ldots$ & $\ldots$ & $\ldots$ \\
\hline 6494.994 & 2.400 & -1.250 & $\ldots$ & $\ldots$ & $\ldots$ & $\ldots$ & $\ldots$ & 132.3 & 109.6 & 179.5 & $\ldots$ & $\ldots$ & 101.8 & 112.1 & $\ldots$ \\
\hline 6498.945 & 0.960 & -4.670 & $\ldots$ & 81.7 & $\ldots$ & $\ldots$ & $\ldots$ & $\ldots$ & $\ldots$ & $\ldots$ & $\ldots$ & $\ldots$ & $\ldots$ & $\ldots$ & 46.3 \\
\hline 6518.373 & 2.830 & -2.400 & $\ldots$ & 68.5 & $\ldots$ & $\ldots$ & $\ldots$ & 38.3 & $\ldots$ & $\ldots$ & $\ldots$ & 72.4 & $\ldots$ & $\ldots$ & $\ldots$ \\
\hline 6569.224 & 4.730 & -0.380 & $\ldots$ & $\ldots$ & $\ldots$ & $\ldots$ & $\ldots$ & $\ldots$ & $\ldots$ & $\ldots$ & $\ldots$ & 91.6 & $\ldots$ & $\ldots$ & $\ldots$ \\
\hline 6593.874 & 2.430 & -2.380 & $\ldots$ & $\ldots$ & $\ldots$ & $\ldots$ & $\ldots$ & $\ldots$ & $\ldots$ & $\ldots$ & $\ldots$ & $\ldots$ & $\ldots$ & 47.4 & $\ldots$ \\
\hline 6597.571 & 4.770 & -0.970 & 40.3 & $\ldots$ & $\ldots$ & $\ldots$ & $\ldots$ & $\ldots$ & $\ldots$ & $\ldots$ & $\ldots$ & $\ldots$ & & & \\
\hline 6608.044 & 2.270 & -3.940 & $\ldots$ & 46.9 & $\ldots$ & $\ldots$ & $\ldots$ & $\ldots$ & $\ldots$ & $\ldots$ & 36.6 & $\ldots$ & $\ldots$ & $\ldots$ & $\ldots$ \\
\hline 6625.039 & 1.010 & -5.280 & $\ldots$ & $\ldots$ & $\ldots$ & $\ldots$ & $\ldots$ & $\ldots$ & $\ldots$ & $\ldots$ & $\ldots$ & 67.8 & $\ldots$ & $\ldots$ & $\ldots$ \\
\hline 6677.997 & 2.690 & -1.400 & $\ldots$ & $\ldots$ & $\ldots$ & 70.3 & $\ldots$ & 91.4 & $\ldots$ & 129.5 & $\ldots$ & $\ldots$ & $\ldots$ & $\ldots$ & 80.9 \\
\hline 6703.576 & 2.760 & -3.060 & $\ldots$ & $\ldots$ & $\ldots$ & $\ldots$ & 57.4 & $\ldots$ & $\ldots$ & $\ldots$ & 39.5 & $\ldots$ & $\ldots$ & $\ldots$ & $\ldots$ \\
\hline
\end{tabular}

Notes. Lines listed twice were measured in two orders with overlapping wavelength coverage.

(This table is available in its entirety in a machine-readable form in the online journal. A portion is shown here for guidance regarding its form and content.)

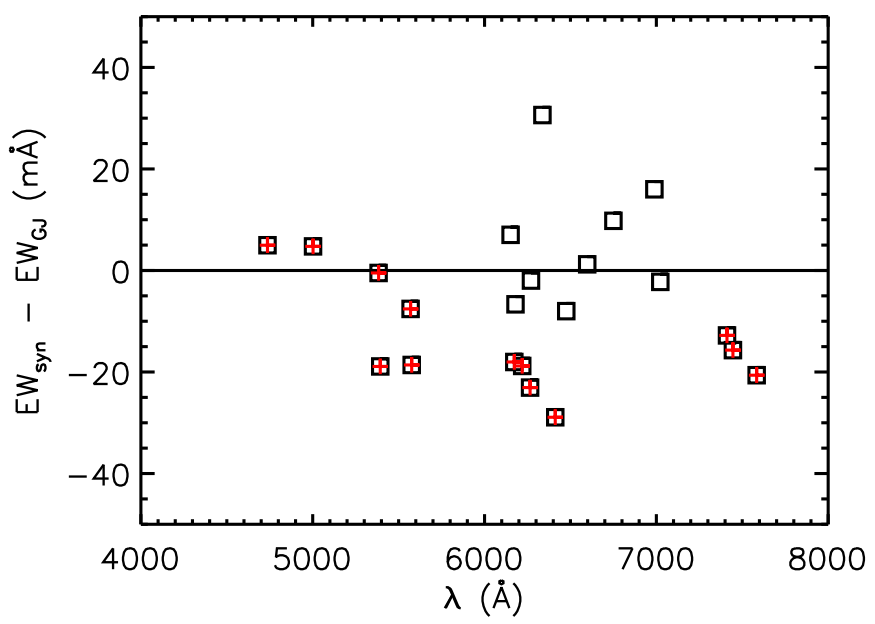

Figure 7. Difference in the EWs measured with GETJOB and the pseudo-EW measured from line synthesis for B384, as a function of wavelength. A solid line is drawn at 0 to guide the eye. Fe lines with $\mathrm{EWs}>100 \mathrm{~m} \AA$ are highlighted in red.

(A color version of this figure is available in the online journal.)

diagnostics for different CMD ages. As an example we show the old (15 Gyr) and young (1 Gyr) solutions for B384 in Figure 9. In the top panels the EW solutions are shown, and we see that the 1 Gyr solution is preferred because there is no dependence of the Fe abundance on wavelength, while for the 15 Gyr solution there is a trend of increasing $\mathrm{Fe}$ abundance with wavelength. In the bottom panels, we show the synthesis solutions, and because in both cases the Fe abundance of the strong, red lines decreased, the old 15 Gyr now exhibits no trend in Fe abundance with wavelength, and is therefore now the better solution; the 1 Gyr solution now has a trend of decreasing Fe abundance with wavelength.

As a final summary, we show the $[\mathrm{Fe} / \mathrm{H}]$ results as a function of $v_{\sigma}$ for the entire M31 sample in Figure 10. In

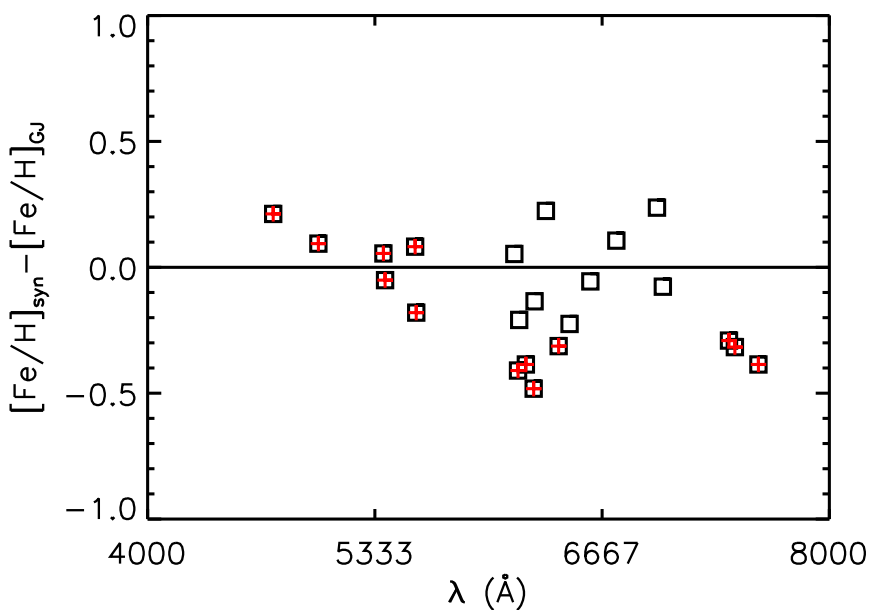

Figure 8. Difference in the $[\mathrm{Fe} / \mathrm{H}]$ obtained using EWs from GETJOB and the $[\mathrm{Fe} / \mathrm{H}]$ measured from line synthesis for $\mathrm{B} 384$, as a function of wavelength. A solid line is drawn at 0 to guide the eye. Fe lines with EWs $>100 \mathrm{~m} \AA$ are highlighted in red.

(A color version of this figure is available in the online journal.)

red, we highlight GCs that meet the criteria for requiring line synthesis analysis $\left([\mathrm{Fe} / \mathrm{H}]>-0.3, v_{\sigma}>15 \mathrm{~km} \mathrm{~s}^{-1}\right)$. In purple, we show the GCs where line synthesis was preferred for $\mathrm{S} / \mathrm{N}$ reasons. In blue, we show the GCs where line synthesis was necessary to distinguish between young and old ages. The five GCs from C09 are shown as black diamonds, demonstrating that they are outside of the regime where line synthesis is necessary.

\section{CHEMICAL ABUNDANCE AND AGE RESULTS}

In this work, we present the Fe abundance analysis results for 26 GCs. The EWs and line parameters of the 15 GCs for which the analysis was performed with GETJOB are listed in Table 4; 

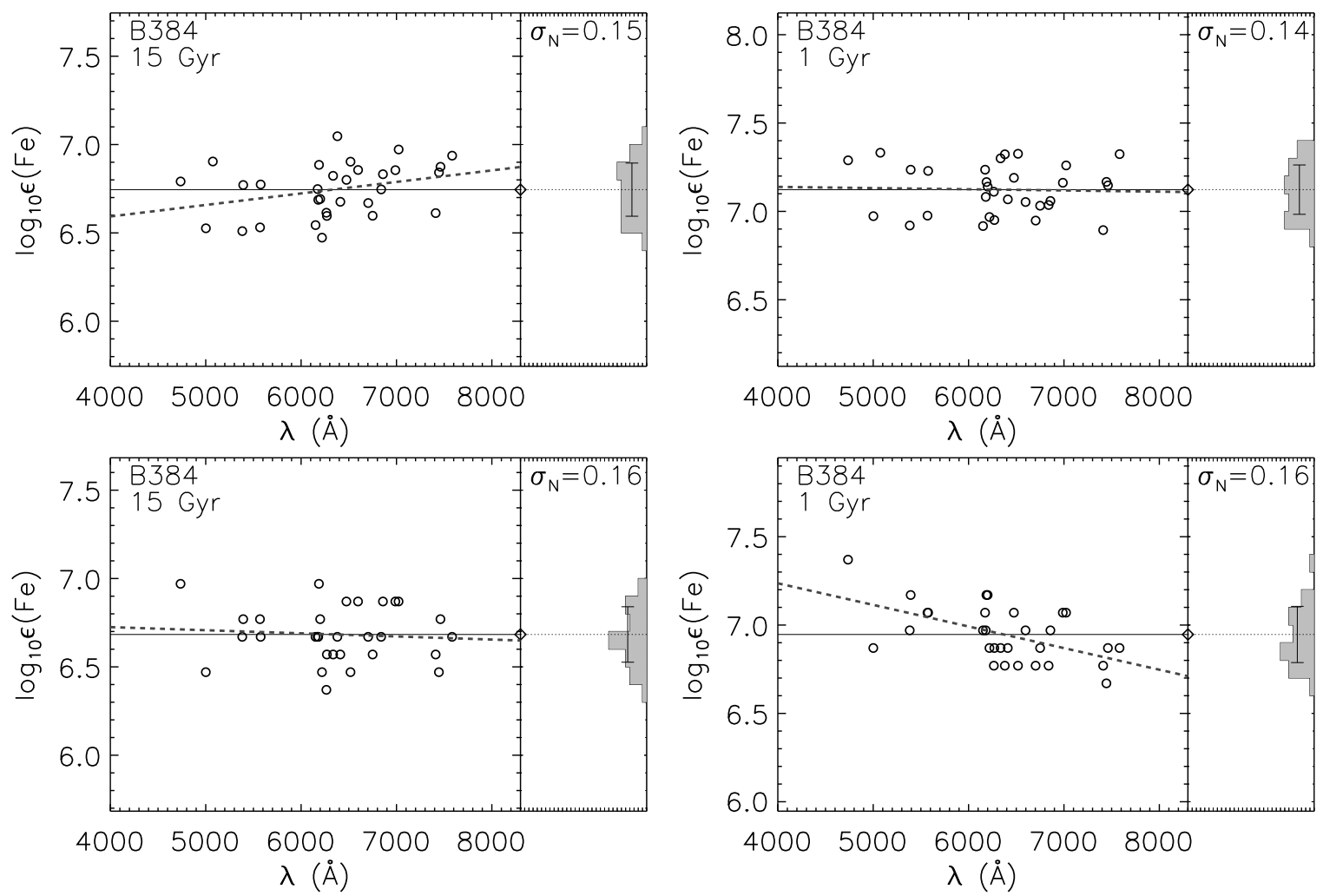

Figure 9. Individual Fe line abundances as a function of wavelength for B384. The top panels are the results using EWs measured in GETJOB, and the bottom panels show the results using line synthesis. In both cases, an old (15 Gyr) solution is shown on the left and a young (1 Gyr) solution in shown on the right. Solid lines show the mean abundance from all lines, and the dashed line shows a linear least-squares fit. Using GETJOB, the young solution is preferred, while with line synthesis the old solution is preferred. More details can be found in the text.

Table 5

M31 GC Fe I Synthesis Abundances

\begin{tabular}{|c|c|c|c|c|c|c|c|c|c|c|c|}
\hline $\begin{array}{l}\lambda \\
(\AA)\end{array}$ & B012 & B029 & B034 & B110 & B163 & B171 & B182 & B193 & B225 & B384 & B457 \\
\hline 6297.799 & $\ldots$ & 6.81 & $\ldots$ & $\ldots$ & 6.53 & $\ldots$ & $\ldots$ & $\ldots$ & $\ldots$ & $\ldots$ & 6.62 \\
\hline 6322.694 & $\ldots$ & $\ldots$ & $\ldots$ & 6.80 & $\ldots$ & 7.03 & $\ldots$ & $\ldots$ & $\ldots$ & $\ldots$ & 6.81 \\
\hline 6335.337 & 5.86 & $\ldots$ & $\ldots$ & $\ldots$ & 7.03 & 7.33 & $\ldots$ & $\ldots$ & $\ldots$ & $\ldots$ & 6.02 \\
\hline 6336.830 & 5.66 & 7.01 & $\ldots$ & $\ldots$ & 6.83 & 7.03 & $\ldots$ & $\ldots$ & $\ldots$ & 6.62 & 5.92 \\
\hline 6355.035 & $\ldots$ & $\ldots$ & $\ldots$ & $\ldots$ & 7.13 & $\ldots$ & $\ldots$ & $\ldots$ & $\ldots$ & $\ldots$ & $\ldots$ \\
\hline 6380.750 & $\ldots$ & $\ldots$ & $\ldots$ & $\ldots$ & 7.13 & 7.53 & $\ldots$ & $\ldots$ & 6.70 & 6.62 & $\ldots$ \\
\hline 6411.658 & 5.66 & $\ldots$ & $\ldots$ & $\ldots$ & 7.03 & 6.83 & $\ldots$ & $\ldots$ & 6.70 & 6.62 & 6.42 \\
\hline 6419.956 & $\ldots$ & $\ldots$ & 6.93 & $\ldots$ & & & $\ldots$ & $\ldots$ & $\ldots$ & $\ldots$ & \\
\hline 6475.632 & $\ldots$ & 7.01 & 6.83 & 6.90 & 7.03 & 7.43 & 6.99 & 7.42 & $\ldots$ & 6.92 & 6.42 \\
\hline 6481.878 & $\ldots$ & 7.11 & $\ldots$ & $\ldots$ & $\ldots$ & $\ldots$ & $\ldots$ & $\ldots$ & $\ldots$ & $\ldots$ & $\ldots$ \\
\hline 6518.373 & $\ldots$ & 6.91 & $\ldots$ & $\ldots$ & $\ldots$ & 7.23 & $\ldots$ & $\ldots$ & $\ldots$ & 6.52 & 6.22 \\
\hline 6569.224 & $\ldots$ & $\ldots$ & $\ldots$ & $\ldots$ & 7.23 & 7.33 & $\ldots$ & $\ldots$ & $\ldots$ & $\ldots$ & $\ldots$ \\
\hline 6597.571 & $\ldots$ & $\ldots$ & $\ldots$ & $\ldots$ & $\ldots$ & $\ldots$ & $\ldots$ & $\ldots$ & $\ldots$ & 6.82 & $\ldots$ \\
\hline 6703.576 & $\ldots$ & 6.91 & 6.53 & 6.70 & 7.03 & 6.93 & $\ldots$ & $\ldots$ & $\ldots$ & 6.62 & $\ldots$ \\
\hline
\end{tabular}

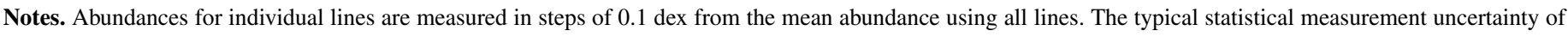

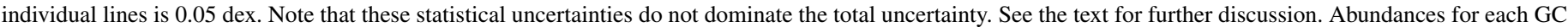
are calculated using its CMD solution that has the oldest age.

(This table is available in its entirety in a machine-readable form in the online journal. A portion is shown here for guidance regarding its form and content.)

and for the remaining $11 \mathrm{GCs}$ that were analyzed with line synthesis, we present the $\mathrm{Fe}$ abundance measured for each line in Table 5. The final $[\mathrm{Fe} / \mathrm{H}]$ and age results and their accompanying uncertainties for the $31 \mathrm{GCs}$ in our full sample are presented in Table 6; the five GCs analyzed previously in C09 are separated at the end of the table for clarity. Table 6 also lists the number of Fe I lines used in the analysis and whether the final results were obtained with an EW analysis or synthesis analysis (SYN).
The last column denotes whether the final abundances were calculated with solar-scaled (S) or $\alpha$-enhanced (A) isochrones and stellar atmospheres. This decision is based on the $\alpha$ element abundances measured for Ca I, Si I, Ti I, and Ti II presented in Section 4.3. We require the averaged [X/Fe] ratio of all of the $\alpha$ elements to be $>+0.15$ for $\alpha$-enhanced calculations to be used instead of scaled-solar. We also present the final Fe solutions as a function of wavelength for the $26 \mathrm{GCs}$ analyzed in this work 
Table 6

M31 GC Ages and Fe I Abundances

\begin{tabular}{|c|c|c|c|c|c|c|c|c|c|}
\hline Name & $\begin{array}{l}\text { Age } \\
\text { (Gyr) }\end{array}$ & $\begin{array}{l}\Delta \text { Age } \\
(\mathrm{Gyr})\end{array}$ & {$[\mathrm{Fe} \mathrm{I} / \mathrm{H}]$} & $N^{\mathrm{a}}$ & $\sigma_{\mathrm{N}}^{\mathrm{b}}$ & $\sigma_{\text {Age }}{ }^{\mathrm{c}}$ & $\sigma_{T}^{\mathrm{d}}$ & $\mathrm{EW} / \mathrm{SYN}^{\mathrm{e}}$ & $\alpha^{\mathrm{f}}$ \\
\hline B006-G058 & 12.5 & 2.5 & -0.73 & 30 & 0.22 & 0.07 & 0.08 & EW & A \\
\hline B012-G064 & 11.5 & 1.5 & -1.61 & 30 & 0.21 & 0.01 & 0.04 & SYN & A \\
\hline B029-G090 & 2.1 & 0.9 & -0.43 & 33 & 0.11 & 0.11 & 0.11 & SYN & A \\
\hline B034-G096 & 12.5 & 2.5 & -0.75 & 24 & 0.14 & 0.05 & 0.06 & SYN & A \\
\hline B048-G110 & 12.5 & 2.5 & -0.85 & 46 & 0.17 & 0.07 & 0.07 & EW & A \\
\hline B088-G150 & 14.0 & 1.0 & -1.71 & 28 & 0.22 & 0.02 & 0.05 & EW & A \\
\hline B110-G172 & 6.5 & 3.5 & -0.66 & 18 & 0.10 & 0.04 & 0.05 & SYN & A \\
\hline B171-G222 & 12.5 & 2.5 & -0.45 & 43 & 0.19 & 0.06 & 0.07 & SYN & A \\
\hline B163-G217 & 11.5 & 1.5 & -0.49 & 41 & 0.18 & 0.03 & 0.04 & SYN & A \\
\hline B182-G233 & 12.5 & 2.5 & -1.04 & 14 & 0.18 & 0.07 & 0.09 & SYN & A \\
\hline B193-G244 & 8.0 & 5.0 & -0.16 & 14 & 0.12 & 0.04 & 0.05 & SYN & A \\
\hline B225-G280 & 10.0 & 3.0 & -0.66 & 16 & 0.13 & 0.03 & 0.05 & SYN & A \\
\hline B232-G286 & 14.0 & 1.0 & -1.77 & 27 & 0.23 & 0.06 & 0.08 & EW & A \\
\hline B235-G297 & 12.5 & 2.5 & -0.86 & 55 & 0.19 & 0.06 & 0.07 & EW & A \\
\hline B240-G302 & 12.5 & 2.5 & -1.54 & 58 & 0.13 & 0.04 & 0.04 & EW & A \\
\hline B311-G033 & 14.0 & 1.0 & -1.71 & 23 & 0.19 & 0.04 & 0.06 & EW & A \\
\hline B312-G035 & 12.5 & 2.5 & -1.06 & 74 & 0.22 & 0.02 & 0.03 & EW & A \\
\hline B383-G318 & 12.5 & 2.5 & -0.78 & 34 & 0.16 & 0.04 & 0.05 & EW & A \\
\hline B384-G319 & 6.5 & 3.5 & -0.63 & 29 & 0.14 & 0.11 & 0.11 & SYN & A \\
\hline B403-G348 & 11.0 & 1.0 & -0.80 & 49 & 0.16 & 0.03 & 0.04 & EW & A \\
\hline B457-G097 & 11.0 & 4.0 & -1.23 & 32 & 0.25 & 0.04 & 0.06 & SYN & A \\
\hline B514-MCGC4 & 14.0 & 1.0 & -1.74 & 72 & 0.17 & 0.04 & 0.04 & EW & A \\
\hline G327-MVI & 11.5 & 1.5 & -1.65 & 36 & 0.25 & 0.06 & 0.07 & EW & A \\
\hline G002 & 11.5 & 1.5 & -1.63 & 64 & 0.16 & 0.04 & 0.04 & EW & $\mathrm{S}$ \\
\hline MCGC5-H10 & 12.5 & 2.5 & -1.45 & 57 & 0.10 & 0.02 & 0.02 & EW & A \\
\hline MGC1 & 11.5 & 1.5 & -1.56 & 24 & 0.09 & 0.02 & 0.03 & EW & A \\
\hline \multicolumn{10}{|c|}{ GCs analyzed in C09 } \\
\hline B045-G108 & 12.5 & 2.5 & -0.94 & 49 & 0.22 & 0.03 & 0.04 & EW & $\mathrm{A}$ \\
\hline B358-G219 & 12.5 & 2.5 & -2.21 & 47 & 0.21 & 0.03 & 0.04 & EW & A \\
\hline B381-G315 & 12.5 & 2.5 & -1.17 & 61 & 0.17 & 0.03 & 0.04 & EW & A \\
\hline B386-G322 & 11.0 & 4.0 & -1.14 & 35 & 0.16 & 0.03 & 0.04 & EW & A \\
\hline B405-G351 & 12.5 & 2.5 & -1.33 & 42 & 0.26 & 0.03 & 0.05 & EW & A \\
\hline
\end{tabular}

Notes. Results from C09 are reproduced here for completeness.

a Number of Fe I lines measured.

b Statistical error of the mean Fe I abundance.

${ }^{\mathrm{c}}$ Dependence of the final abundance on the age of the CMD.

${ }^{\mathrm{d}}$ Final total uncertainty in abundance, $\sigma_{T}=\left(\left(\sigma_{\mathrm{N}} / \sqrt{N-1}\right)^{2}+\sigma_{\text {Age }}^{2}\right)(1 / 2)$.

e Denotes whether the final analysis was performed with GETJOB (EW) or line synthesis (SYN).

${ }^{\mathrm{f}}$ Denotes whether solar-scaled (S) or $\alpha$-enhanced (A) isochrones and stellar atmospheres were used in the final analysis.

in Figure 11 for those analyzed with GETJOB and in Figure 12 for those analyzed with line synthesis.

\subsection{Age-Metallicity Relationship}

In Figure 13, we show the age-metallicity relationship for our current sample of M31 GCs. With the exception of B029, all of the GCs are consistent with having ages at least as old as $10 \mathrm{Gyr}$. A handful of the higher metallicity GCs (B110, B193, B384) are consistent with either having ages between 5 and 10 Gyr or having a strong contribution to the IL from hot blue horizontal branch (HB) stars. However, the colors for these GCs suggest that it is more likely that these GCs are old with blue HBs (Kang et al. 2012). Finally, we find an intermediate age for one GC (B029) as mentioned in Section 3.2.1, which we discuss in more detail in the following section.

\subsubsection{B029: Evidence for an Intermediate Age}

B029 is the only GC in our current sample for which we find an intermediate age; in this work, we define intermediate age

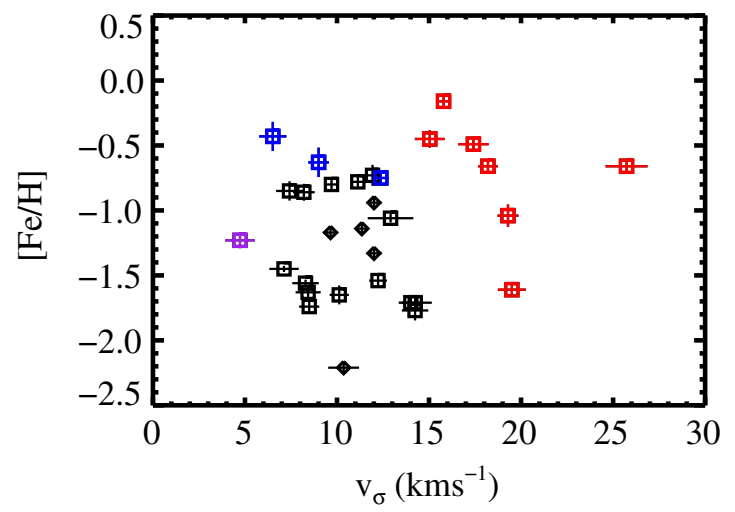

Figure 10. Final $[\mathrm{Fe} / \mathrm{H}]$ plotted against velocity dispersion for the M31 sample. Squares are new GCs in this work, diamonds show GCs from C09. Black symbols show GCs for which the final analysis was performed with GETJOB. Red symbols show GCs where line synthesis was used because the GCs fit the $[\mathrm{Fe} / \mathrm{H}]>-0.3$ or $v_{\sigma}>15 \mathrm{~km} \mathrm{~s}^{-1}$ criteria. Purple and blue symbols show $\mathrm{GCs}$ where synthesis was used because of $\mathrm{S} / \mathrm{N}$ or potentially young ages, respectively.

(A color version of this figure is available in the online journal.) 

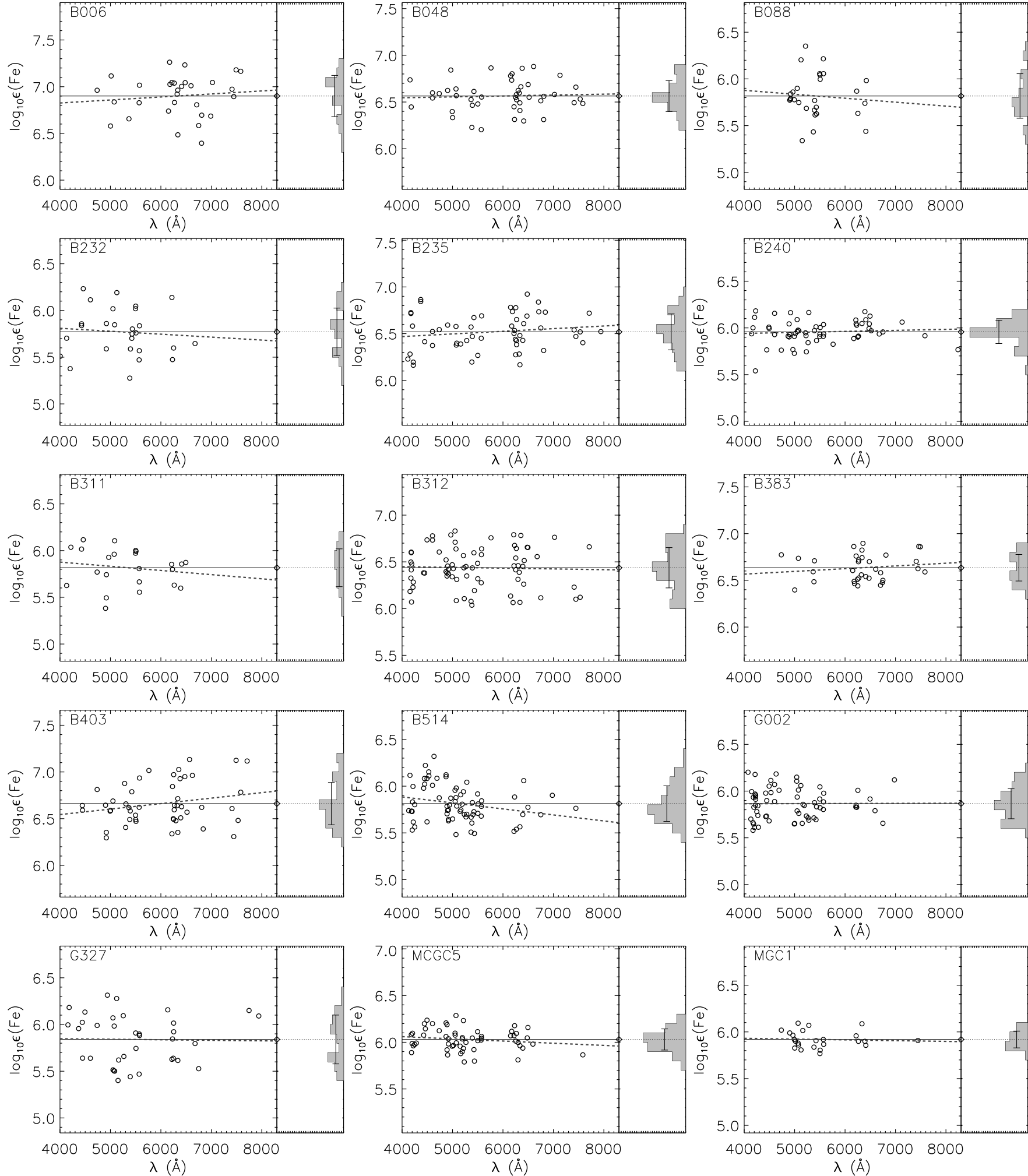

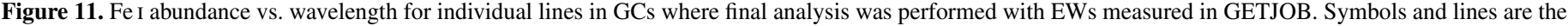
same as in Figure 9. The total range in the $y$ axis is the same in all panels in order to appreciate the range in quality of the solutions.

as having formed between 1 and 5 Gyr ago. As discussed in Section 3.2.1, to check this result, we have performed the Fe I analysis with both EWs and synthesis and we find consistency between the two methods. In addition, we have performed experiments with adding very hot, completely blue HBs to the synthetic CMDs. In performing these tests, we replaced all of the red HB stars with blue $\mathrm{HB}$ stars, while conserving the total $V$ flux on the HB. We can find no "extreme" HB cases that give a more self consistent $\mathrm{Fe}$ I solution across all of the diagnostics than the one obtained with an intermediate age. The diagnostic patterns we find for B029 are similar to what we found for the $\sim 2 \mathrm{Gyr}$ LMC clusters we analyzed in C11, where we demonstrated that 

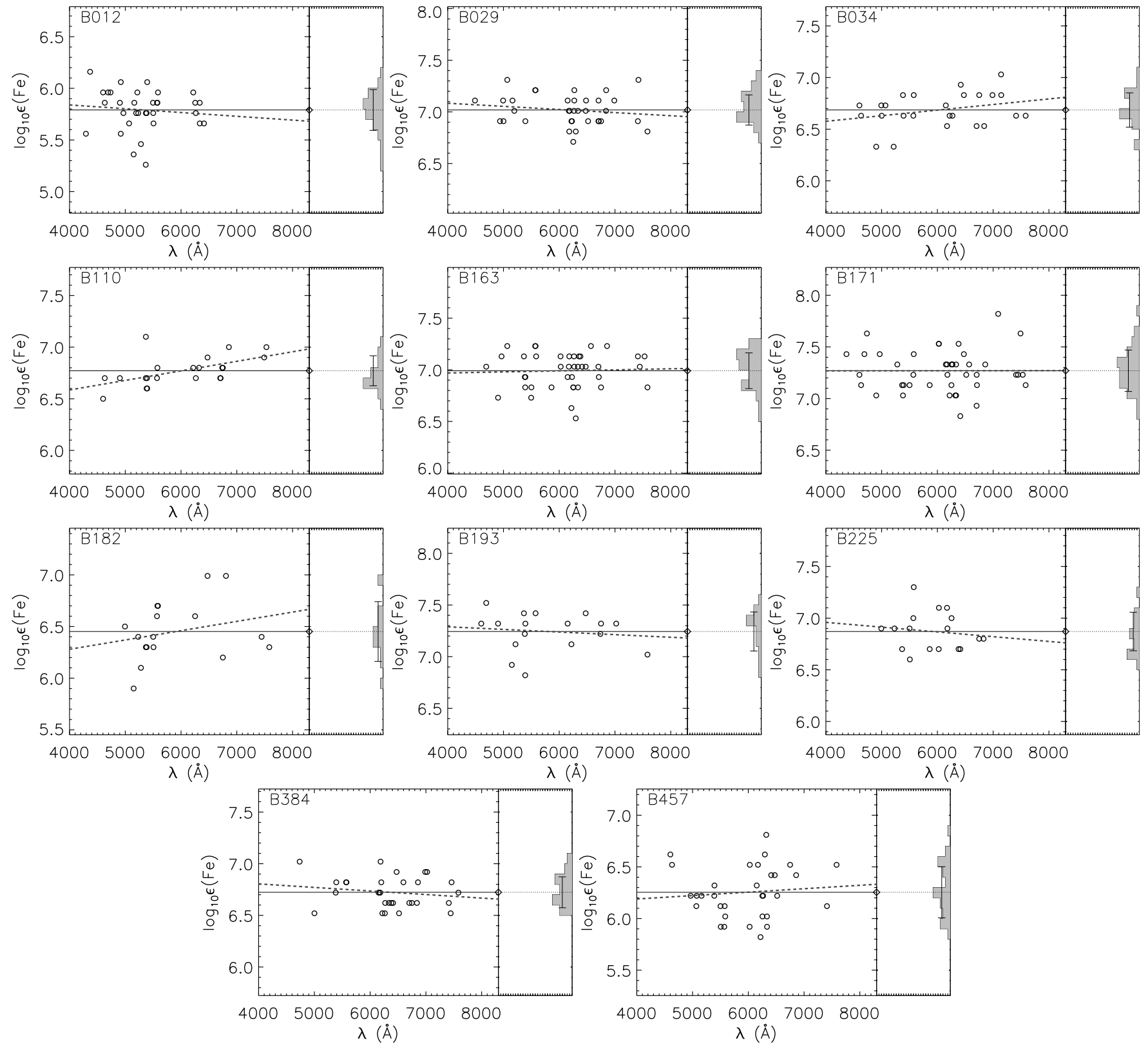

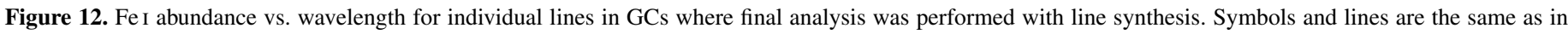
Figure 9. The total range in the $y$ axis is the same in all panels in order to appreciate the range in quality of the solutions.

the Fe line diagnostics are successful in identifying clusters that have ages $<5$ Gyr.

To illustrate the difference evident in our analysis, in Figure 14 we show the analysis diagnostics for an age of $1.6 \mathrm{Gyr}$, an age of $\sim 13 \mathrm{Gyr}$, and an age of $\sim 13 \mathrm{Gyr}$ with an extreme, hot HB. There are several points to take away from Figure 14. First, the $\mathrm{Fe}$ solutions in the top panels, for an age of $1.6 \mathrm{Gyr}$, clearly have a smaller statistical scatter and smaller trends with wavelength, $\mathrm{EW}$, and EP than the solutions in the middle panels for an age of 13 Gyr. We note that it is possible to eliminate the trend in $[\mathrm{Fe} / \mathrm{H}]$ with wavelength by using a $13 \mathrm{Gyr} \mathrm{CMD}$ with a completely blue HB, as shown in the bottom panels. However, the statistical scatter and behavior of $[\mathrm{Fe} / \mathrm{H}]$ with $\mathrm{EW}$ and $\mathrm{EP}$ in the extreme blue HB case are still not as good as in the $1.6 \mathrm{Gyr}$ case. Prior experience with our training set GCs suggests low statistical scatter is a critical characteristic of a good solution (McWilliam \& Bernstein 2008; Cameron 2009;

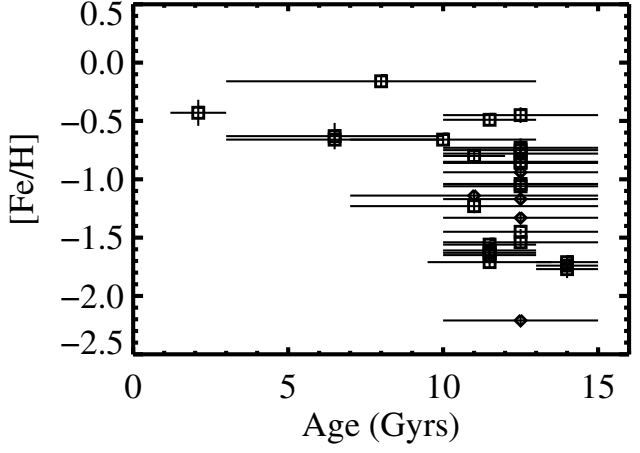

Figure 13. Age-metallicity relationship for the M31 GC sample. GCs from C09 are shown as diamonds. All GCs, with the exception of one, have solutions consistent with ages of $\geqslant 10 \mathrm{Gyr}$. 

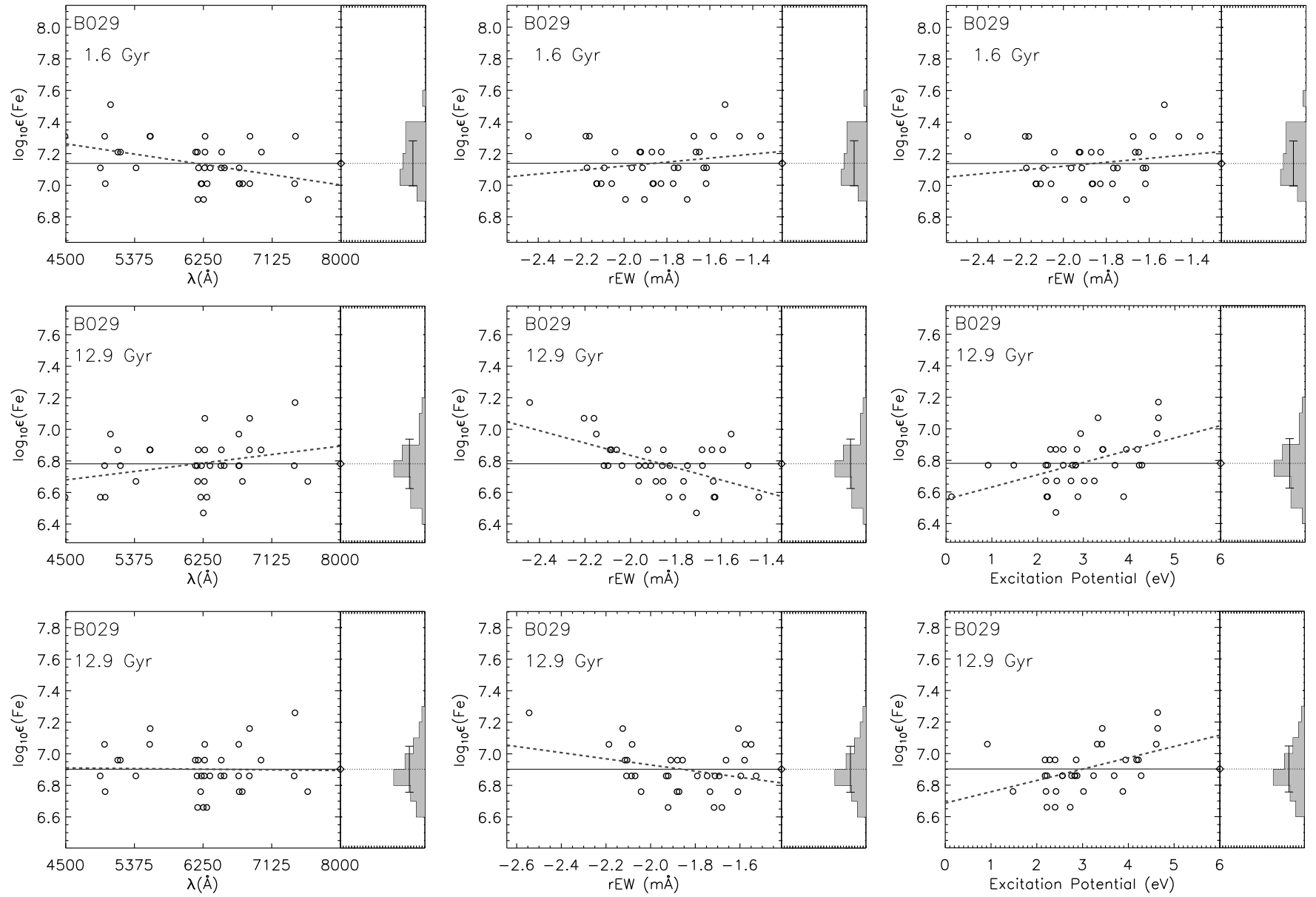

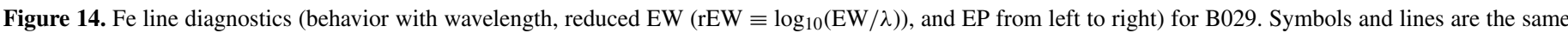

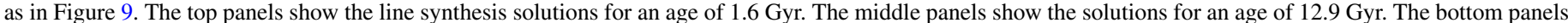

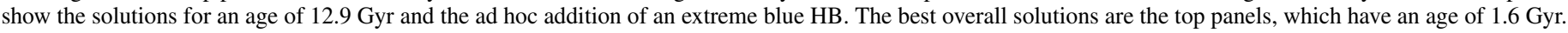

Colucci et al. 2011, 2014). Therefore, we conclude that overall the best solutions for $\mathrm{B} 029$ are for ages of $\sim 2 \mathrm{Gyr}$ and a relatively high overall metallicity of $[\mathrm{Fe} / \mathrm{H}]=-0.43$.

The only definitive way to confirm an intermediate age for B029 is to obtain a deep Hubble Space Telescope CMD that reaches the turnoff, which unfortunately is not available and would require several days of integration time (e.g., Brown et al. 2004). There are other indirect, non-spectroscopic strategies for arguing for or against intermediate ages for extragalactic GCs when CMDs that reach the turnoff are unavailable. We consider three common consistency checks in this section, but note that age dating unresolved GCs has a long, extensive and sometimes conflicting history.

First, the simplest consistency check is to consider integrated optical colors. Unfortunately, B029 is significantly reddened, likely because it is projected onto the outer disk of M31. The $E(B-V)$ in the literature vary quite a bit; Fan et al. (2008) find $E(B-V)=0.12$, while Caldwell et al. (2011) find $E(B-V)=$ 0.27 . The differences in these $E(B-V)$ values alone make conclusions drawn from colors ambiguous. As an example, in Figure 15, we show the predicted $(B-V)_{\mathrm{o}}$ from the Teramo isochrones used in our analysis compared to the observed $B-V$ for B029. Figure 15 shows that the lower $E(B-V)$ value of Fan et al. (2008) is consistent with an old age for B029, but that the higher of Caldwell et al. (2011) is consistent with the younger age we determine (however, we note that Caldwell et al. (2011) find an old age for B029 from low-resolution spectroscopy). We

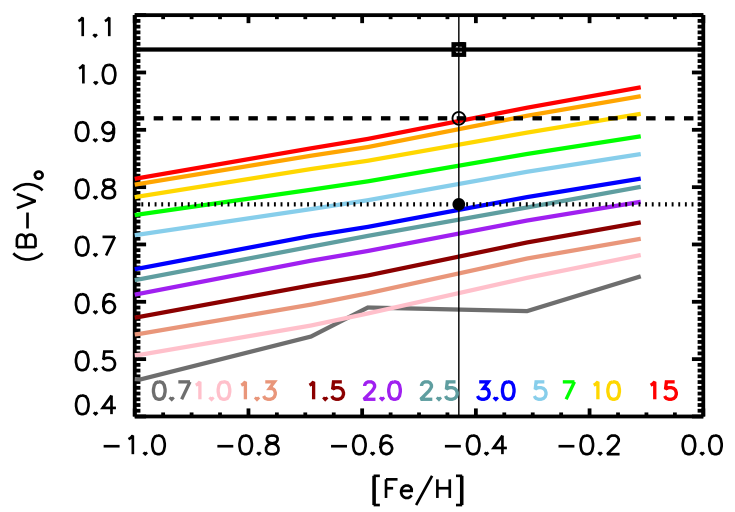

Figure 15. Predicted integrated color as a function of $[\mathrm{Fe} / \mathrm{H}]$ and age, as calculated from the Teramo isochrones we use in our analysis. To guide the eye, a vertical solid line marks the $[\mathrm{Fe} / \mathrm{H}]$ solution we find for $\mathrm{B} 029$. The observed $B-V$ color of B029 from the Revised Bologna Catalog (Galleti et al. $2004)$ is shown as a horizontal solid line. The dashed line shows the reddening corrected color of B029, using the $E(B-V)=0.12$ from Fan et al. (2008). The dotted line shows the corrected color using the $E(B-V)=0.27$ from Caldwell et al. (2011). The latter is consistent with the age range we derive.

(A color version of this figure is available in the online journal.)

conclude that an intermediate age for B029 cannot be ruled out by the observed optical colors.

Second, $M / L$ ratios are often investigated under the assumption that intermediate age GCs will have larger $M / L$ s than old 
Table 7

M31 GC Line Abundances

\begin{tabular}{|c|c|c|c|c|c|c|c|c|c|c|c|c|}
\hline \multirow[t]{2}{*}{ Species } & \multirow{2}{*}{$\begin{array}{c}\lambda \\
(\AA)\end{array}$} & \multirow{2}{*}{$\begin{array}{c}\text { EP } \\
(\mathrm{eV})\end{array}$} & \multirow[t]{2}{*}{$\log g f$} & \multicolumn{9}{|c|}{$12+\log (\mathrm{X} / \mathrm{H})$} \\
\hline & & & & B006 & B012 & В029 & B034 & B045 & B048 & B088 & B110 & B163 \\
\hline $\mathrm{Na} I$ & 5682.650 & 2.100 & -0.700 & 6.22 & 4.90 & 6.24 & 5.89 & 5.43 & $\ldots$ & $\ldots$ & $\ldots$ & 6.37 \\
\hline $\mathrm{Na} I$ & 5688.220 & 2.100 & -0.460 & 6.32 & 5.20 & 6.24 & 6.09 & 5.53 & $\ldots$ & $\ldots$ & $\ldots$ & 6.57 \\
\hline $\mathrm{Na} I$ & 6154.230 & 2.100 & -1.570 & 6.12 & 5.39 & 6.34 & 5.89 & 5.53 & $\ldots$ & $\ldots$ & $\ldots$ & $\ldots$ \\
\hline $\mathrm{Na} \mathrm{I}$ & 6160.753 & 2.100 & -1.270 & $\ldots$ & 5.39 & $\ldots$ & $\ldots$ & $\ldots$ & $\ldots$ & $\ldots$ & $\ldots$ & $\ldots$ \\
\hline $\mathrm{Mg}_{\mathrm{I}}$ & 4167.277 & 4.346 & -0.995 & $\ldots$ & $\ldots$ & $\ldots$ & 7.10 & $\ldots$ & 6.84 & $\ldots$ & $\ldots$ & $\ldots$ \\
\hline $\mathrm{Mg}_{\mathrm{I}}$ & 4351.921 & 4.346 & -0.520 & $\ldots$ & 6.55 & $\ldots$ & $\ldots$ & $\ldots$ & $\ldots$ & $\ldots$ & $\ldots$ & $\ldots$ \\
\hline Mg I & 4571.102 & 0.000 & -5.569 & 7.38 & $\ldots$ & $\ldots$ & 7.00 & 6.89 & 6.54 & 5.36 & $\ldots$ & $\ldots$ \\
\hline $\operatorname{Mg} I_{I}$ & 4703.003 & 4.346 & -0.377 & 7.38 & $\ldots$ & $\ldots$ & $\ldots$ & 6.59 & 6.44 & 5.46 & $\ldots$ & $\ldots$ \\
\hline
\end{tabular}

(This table is available in its entirety in a machine-readable form in the online journal. A portion is shown here for guidance regarding its form and content.)

GCs because the stellar populations are less evolved. Strader et al. (2011) calculated $M / L$ ratios for a large sample of M31 GCs, and for B029 found $M / L=1.5$ in $V$ and $M / L=0.5$ in $\mathrm{K}$, which is comparable to the rest of their old, high $[\mathrm{Fe} / \mathrm{H}]$ GCs in M31. However, Strader et al. (2011) also find that the behavior of the $M / L$ ratios in GCs does not follow that expected from stellar population models. Similarly, Zaritsky et al. (2012) and Zaritsky et al. (2013) also discuss how $M / L$ ratios of well-studied, resolved GCs do not consistently match current theoretical expectations. Therefore, it's hard to draw definitive conclusions about $\mathrm{GC}$ properties using $M / L$ ratios until their behavior is better understood theoretically.

Third, near-ultraviolet (NUV) and far-ultraviolet (FUV) colors are used in order to distinguish between GCs with young ages and GCs that have very hot HB stars. An old GC with significant numbers of hot $\left(T_{\text {eff }}>10,000 \mathrm{~K}\right) \mathrm{HB}$ stars will be detectable in the FUV, with progressively older ages producing stronger FUV flux (e.g., Lee et al. 2002). Very young GCs ( $\leqslant 0.5 \mathrm{Gyr})$ will also have significant NUV and FUV flux due to hot main sequence stars, but these GCs can be identified by significantly bluer optical colors than what is observed for B029 (Rey et al. 2009). True intermediate age GCs are faint or undetectable in the FUV (Lee \& Worthey 2005). Rey et al. (2009), with an update by Kang et al. (2012), present the GALEX FUV and NUV catalog of M31 GCs. B029 is not detected in either the NUV or FUV, which is consistent with having an intermediate age. However, the high reddening remains a possible explanation for the lack of blue flux, so even this evidence is not particularly definitive.

In summary, we do not find compelling independent evidence to rule out an intermediate age for B029. Because Balmer lines are temperature sensitive, they have historically been the primary spectroscopic age indicator. However, ages derived from Balmer line strengths are notoriously problematic because the same line depths can be achieved for true intermediate age clusters and old clusters that have significant numbers of blue HB stars (e.g., Worthey 1994; Schiavon et al. 2004; Percival \& Salaris 2011). A strength of our analysis compared to other spectroscopic techniques is that we use only unsaturated $\mathrm{Fe}$ lines to constrain the ages of the clusters so that we can avoid the degeneracies between hot main-sequence and hot HB stars. Instead, unsaturated Fe lines are principally sensitive to the temperature of the red giant branch (RGB), AGB, and turnoff stars (see discussions in MB08, C09, C11). Therefore, this is the first evidence for the existence of an intermediate age cluster in M31 that does not solely rely on integrated colors or Balmer line absorption

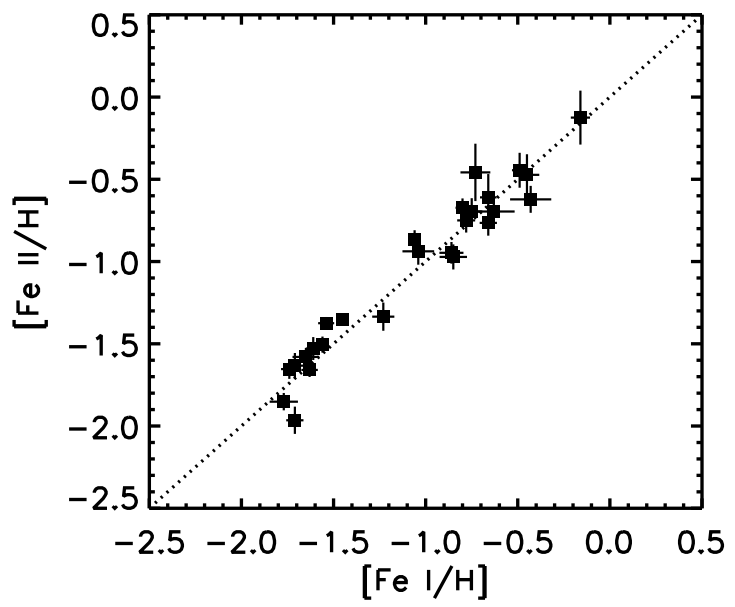

Figure 16. Comparison of the mean abundances measured from Fe II lines and the mean abundances measured from Fe I lines. The dotted line shows 1:1 correspondence.

from line indexes (Barmby \& Huchra 2000; Jiang et al. 2003; Beasley et al. 2004, 2005; Puzia et al. 2005). Additional highresolution abundance analyses of GCs that are not detected in GALEX would be interesting to further investigate the intermediate age population of GCs in M31.

\subsection{Abundances from Fe II Lines}

In addition to abundance measurements from $\mathrm{Fe}$ I lines, which we use to constrain the age and metallicity of the GCs, we are also able to measure abundances from Fe II lines. We have measured all of the Fe II abundances using spectral synthesis and $\chi^{2}$ minimization, since there are fewer trusted transitions and they are often significantly more blended than the Fe I lines. For consistency, we have re-measured the Fe II lines for GCs from C09 using spectral synthesis. The final abundance of each Fe II line, as well as the adopted line parameters, are given in Table 7. The mean $[\mathrm{Fe}$ II $/ \mathrm{H}]$ abundances are listed in Table 8 along with the statistical error and the number of Fe II lines used in the analysis. With line synthesis, on average we are able to measure $\sim 10 \mathrm{Fe}$ II lines for each GC, which is an improvement over what we were able to measure in $\mathrm{C} 09$, where we averaged 3-4 Fe II lines per GC. We find that the abundances measured from Fe II lines are consistent with the abundances measured from $\mathrm{Fe}$ I lines, as shown in Figure 16. The Fe II and Fe I abundances all agree to within $2 \sigma$, with most agreeing within $1 \sigma$. The excellent agreement of the Fe II abundances is independent confirmation of the accuracy of our measurements. 
Table 8

M31 GC Mean Abundances

\begin{tabular}{|c|c|c|c|c|c|c|c|c|c|c|c|c|c|c|c|c|}
\hline $\begin{array}{l}\text { Name } \\
\text { (1) }\end{array}$ & $\begin{array}{c}{[\mathrm{Fe} \mathrm{II} / \mathrm{H}]} \\
(2)\end{array}$ & $\begin{array}{c}\sigma_{\mathrm{Fe} \mathrm{II}} \\
(3)\end{array}$ & $\begin{array}{c}N_{\mathrm{Fe} \text { II }} \\
(4)\end{array}$ & $\begin{array}{c}\sigma_{\mathrm{A}, \mathrm{Fe} \text { II }} \\
(5)\end{array}$ & $\begin{array}{c}{[\mathrm{Na} I / \mathrm{Fe}]} \\
(6)\end{array}$ & $\begin{array}{c}\sigma_{\mathrm{Na} \mathrm{I}} \\
(7)\end{array}$ & $\begin{array}{c}N_{\mathrm{NaI}} \\
(8)\end{array}$ & $\begin{array}{c}\sigma_{\mathrm{A}, \mathrm{Na}} \\
(9)\end{array}$ & $\begin{array}{c}{[\mathrm{Mg} \mathrm{I} / \mathrm{Fe}]} \\
(10)\end{array}$ & $\begin{array}{c}\sigma_{\mathrm{MgI}_{\mathrm{I}}} \\
(11)\end{array}$ & $\begin{array}{c}N_{\mathrm{Mg}_{\mathrm{I}}} \\
(12)\end{array}$ & $\begin{array}{c}\sigma_{\mathrm{A}, \mathrm{Mg}} \\
(13)\end{array}$ & $\begin{array}{c}{[\mathrm{Al} \mathrm{I} / \mathrm{Fe}]} \\
(14)\end{array}$ & $\begin{array}{l}\sigma_{\mathrm{Al} \mathrm{I}} \\
(15)\end{array}$ & $\begin{array}{l}N_{\mathrm{Al} \text { I }} \\
(16)\end{array}$ & $\begin{array}{c}\sigma_{\mathrm{A}, \mathrm{Al}} \\
(17)\end{array}$ \\
\hline B006-G058 & -0.46 & 0.04 & 12 & 0.17 & 0.52 & 0.04 & 3 & 0.02 & 0.32 & 0.03 & 5 & 0.03 & 0.62 & 0.13 & 2 & 0.10 \\
\hline B012-G064 & -1.53 & 0.06 & 9 & 0.03 & 0.62 & 0.13 & 4 & 0.00 & -0.08 & 0.07 & 2 & 0.04 & $\ldots$ & $\ldots$ & $\ldots$ & $\ldots$ \\
\hline B029-G090 & -0.62 & 0.06 & 11 & 0.06 & 0.48 & 0.08 & 3 & 0.07 & 0.17 & 0.08 & 3 & 0.05 & 0.38 & 0.14 & 2 & 0.11 \\
\hline B034-G096 & -0.70 & 0.06 & 11 & 0.05 & 0.47 & 0.08 & 3 & 0.00 & 0.21 & 0.08 & 5 & 0.04 & 0.50 & 0.10 & 1 & 0.00 \\
\hline B045-G108 & -0.84 & 0.06 & 11 & 0.07 & 0.12 & 0.04 & 3 & 0.07 & 0.04 & 0.15 & 4 & 0.05 & 0.10 & 0.07 & 2 & 0.07 \\
\hline B048-G110 & -0.97 & 0.04 & 16 & 0.06 & $\ldots$ & $\ldots$ & $\ldots$ & $\ldots$ & 0.10 & 0.08 & 5 & 0.11 & 0.40 & 0.07 & 2 & 0.07 \\
\hline B088-G150 & -1.97 & 0.07 & 6 & 0.04 & $\ldots$ & $\ldots$ & $\ldots$ & $\ldots$ & -0.48 & 0.00 & 3 & 0.02 & $\ldots$ & $\ldots$ & $\ldots$ & $\ldots$ \\
\hline B110-G172 & -0.77 & 0.06 & 8 & 0.05 & $\ldots$ & $\ldots$ & $\ldots$ & $\ldots$ & 0.40 & 0.10 & 1 & 0.00 & $\ldots$ & $\ldots$ & $\ldots$ & $\ldots$ \\
\hline B163-G217 & -0.45 & 0.10 & 4 & 0.04 & 0.72 & 0.07 & 2 & 0.04 & 0.25 & 0.10 & 1 & 0.07 & 0.50 & 0.00 & 2 & 0.00 \\
\hline B171-G222 & -0.47 & 0.12 & 6 & 0.03 & 0.73 & 0.08 & 3 & 0.00 & 0.18 & 0.17 & 4 & 0.02 & 0.38 & 0.21 & 2 & 0.04 \\
\hline B182-G233 & -0.94 & 0.08 & 7 & 0.01 & $\ldots$ & $\ldots$ & $\ldots$ & $\ldots$ & 0.35 & 0.10 & 1 & 0.07 & $\ldots$ & $\ldots$ & $\ldots$ & $\ldots$ \\
\hline B193-G244 & -0.12 & 0.12 & 5 & 0.11 & $\ldots$ & $\ldots$ & $\ldots$ & $\ldots$ & 0.40 & 0.10 & 1 & 0.00 & $\ldots$ & $\ldots$ & $\ldots$ & $\ldots$ \\
\hline B225-G280 & -0.61 & 0.14 & 2 & 0.01 & $\ldots$ & $\ldots$ & $\ldots$ & $\ldots$ & 0.45 & 0.10 & 1 & 0.07 & $\ldots$ & $\ldots$ & $\ldots$ & $\ldots$ \\
\hline B232-G286 & -1.85 & 0.04 & 9 & 0.03 & 0.35 & 0.07 & 2 & 0.00 & -0.10 & 0.04 & 2 & 0.00 & $\ldots$ & $\ldots$ & $\ldots$ & $\ldots$ \\
\hline B235-G297 & -0.95 & 0.04 & 15 & 0.05 & 0.43 & 0.04 & 3 & 0.05 & -0.01 & 0.09 & 4 & 0.02 & 0.35 & 0.10 & 1 & 0.07 \\
\hline B240-G302 & -1.38 & 0.04 & 14 & 0.01 & 0.23 & 0.07 & 2 & 0.04 & -0.03 & 0.05 & 3 & 0.08 & $\ldots$ & $\ldots$ & $\ldots$ & $\ldots$ \\
\hline B311-G033 & -1.63 & 0.08 & 6 & 0.00 & $\ldots$ & $\ldots$ & $\ldots$ & $\ldots$ & -0.07 & 0.12 & 3 & 0.05 & $\ldots$ & $\ldots$ & $\ldots$ & $\ldots$ \\
\hline B312-G035 & -0.87 & 0.06 & 10 & 0.01 & 0.38 & 0.00 & 2 & 0.04 & 0.00 & 0.14 & 2 & 0.00 & $\ldots$ & $\ldots$ & $\ldots$ & $\ldots$ \\
\hline B358-G219 & -2.35 & 0.04 & 8 & 0.04 & $\ldots$ & $\ldots$ & $\ldots$ & $\ldots$ & -0.03 & 0.21 & 2 & 0.00 & 0.69 & 0.05 & 2 & 0.05 \\
\hline B381-G315 & -1.03 & 0.06 & 11 & 0.00 & 0.23 & 0.11 & 3 & 0.00 & 0.15 & 0.07 & 5 & 0.01 & $\ldots$ & $\ldots$ & $\ldots$ & $\ldots$ \\
\hline B383-G318 & -0.75 & 0.07 & 13 & 0.01 & 0.55 & 0.00 & 3 & 0.07 & 0.24 & 0.07 & 4 & 0.01 & 0.35 & 0.14 & 2 & 0.07 \\
\hline B384-G319 & -0.70 & 0.06 & 14 & 0.08 & 0.33 & 0.04 & 3 & 0.05 & 0.15 & 0.06 & 4 & 0.00 & 0.30 & 0.10 & 1 & 0.00 \\
\hline B386-G322 & -1.11 & 0.05 & 11 & 0.03 & 0.38 & 0.07 & 2 & 0.04 & 0.13 & 0.05 & 5 & 0.01 & $\ldots$ & $\ldots$ & $\ldots$ & $\ldots$ \\
\hline B403-G348 & -0.67 & 0.05 & 15 & 0.03 & 0.38 & 0.07 & 3 & 0.02 & 0.18 & 0.05 & 6 & 0.00 & 0.20 & 0.00 & 2 & 0.00 \\
\hline B405-G351 & -1.35 & 0.07 & 9 & 0.04 & 0.25 & 0.07 & 2 & 0.00 & -0.03 & 0.07 & 5 & 0.01 & $\ldots$ & $\ldots$ & $\ldots$ & $\ldots$ \\
\hline B457-G097 & -1.33 & 0.08 & 10 & 0.02 & 0.07 & 0.14 & 4 & 0.04 & -0.42 & 0.15 & 5 & 0.11 & $\cdots$ & $\ldots$ & $\ldots$ & $\ldots$ \\
\hline B514-MCGC4 & -1.65 & 0.04 & 12 & 0.04 & 0.45 & 0.10 & 1 & 0.07 & -0.16 & 0.07 & 4 & 0.12 & $\ldots$ & $\ldots$ & $\ldots$ & $\ldots$ \\
\hline G002 & -1.66 & 0.03 & 12 & 0.03 & $\ldots$ & $\ldots$ & $\ldots$ & $\ldots$ & -0.55 & 0.07 & 3 & 0.07 & $\cdots$ & $\cdots$ & $\cdots$ & $\cdots$ \\
\hline G327-MVI & -1.58 & 0.05 & 10 & 0.03 & $\ldots$ & $\ldots$ & $\ldots$ & $\ldots$ & 0.02 & 0.10 & 4 & 0.02 & $\ldots$ & $\ldots$ & $\ldots$ & $\ldots$ \\
\hline MCGC5-H10 & -1.35 & 0.04 & 14 & 0.00 & -0.03 & 0.07 & 2 & 0.11 & -0.09 & 0.06 & 5 & 0.02 & $\ldots$ & $\ldots$ & $\ldots$ & $\ldots$ \\
\hline MGC1 & -1.50 & 0.04 & 13 & 0.02 & 0.10 & 0.00 & 2 & 0.00 & 0.09 & 0.15 & 3 & 0.08 & $\ldots$ & $\ldots$ & $\ldots$ & $\ldots$ \\
\hline
\end{tabular}

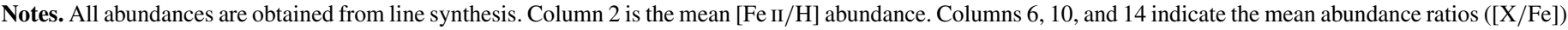

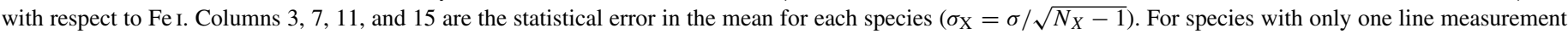

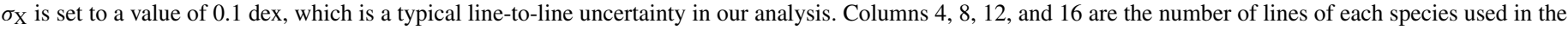
analysis $\left(N_{\mathrm{X}}\right)$. Columns $5,9,13$, and 17 are the uncertainty in the abundance due to the age of the $\mathrm{CMD}\left(\sigma_{\mathrm{A}, \mathrm{X}}\right)$.

\section{3. $\alpha$ Elements}

One goal of this work is to study the $\alpha$-element abundance pattern of M31 GCs, as sampled by the elements $\mathrm{Ca}$, $\mathrm{Si}$, and Ti. These $\alpha$ elements are produced and ejected into the interstellar medium (ISM) in large quantities by type II supernovae (SN II), whose progenitors are massive stars that live and die on short timescales on the order of millions of years (e.g., Woosley \& Weaver 1995). They build up quickly in active star formation epochs. The particular utility of $\alpha$ elements for learning about star formation histories comes from the comparison of their abundances to elements formed on different timescales. The standard comparison is to Fe or Fe-peak elements, which are contributed to the ISM in large quantities on comparatively longer timescales in supernovae type Ia (SN Ia), and in the simplest sense the behavior of the $[\alpha / \mathrm{Fe}]$ in a galaxy lets us infer the rates and timescales of star formation throughout its history. Here we aim to compare the $[\alpha / \mathrm{Fe}]$ abundance pattern in M31 GCs to GCs and stars in the MW in order to constrain the relative strengths and durations of star formation in M31.

We measure abundances for the $\alpha$-elements $\mathrm{Ca}$ I, Si I, Ti I, and Ti II. Note that we also measure Mg I, however, we do not include it here because it is not necessarily mono-metallic in GCs and so is instead included in Section 4.4. All of the $\alpha$-element measurements were made using IL spectral synthesis and $\chi^{2}$ minimization, which enables us to recover more individual lines for each element and with higher precision than the EW analysis we employed in C09. The abundances measured for each line are presented in Table 7 . The final averaged measurements for the $\alpha$ elements for the full M31 sample are listed in Table 9. In Figures 17 and 18, we show examples of the quality of the synthesis fits to $\mathrm{Ca}$ I and $\mathrm{Ti}$ II for three GCs.

The uncertainties in the abundances of the non-Fe elements are calculated in the same way as for $\mathrm{Fe}_{\mathrm{I}}$ when multiple lines are available, which gives an indication of the systematic errors between lines, as well as the uncertainty due to the age of the CMD. A special case occurs when we have only a single measurement of a given species, and therefore no indication of the line-to-line scatter. As an example, we have only one measurement of Si I for B012, and one measurement of $\mathrm{TiI}$ for B088. In these cases we have adopted a typical line-to-line scatter of $0.1 \mathrm{dex}$ as an estimate of the systematic error in the abundance, which is then added in quadrature with the systematic age uncertainty to obtain a total uncertainty.

The lines we measure in the GC IL spectra are all lines that are commonly measured in individual stars. Here we include some additional notes on their behavior in the IL spectra. Our Ca I line list includes 17 lines; the majority of which are 
Table 9

M31 GC Abundances Continued

\begin{tabular}{|c|c|c|c|c|c|c|c|c|c|c|c|c|c|c|c|c|}
\hline $\begin{array}{l}\text { Name } \\
\text { (1) }\end{array}$ & $\begin{array}{c}{[\mathrm{Ca} \mathrm{I} / \mathrm{Fe}]} \\
(2)\end{array}$ & $\begin{array}{c}\sigma_{\mathrm{Ca}} \mathrm{I} \\
(3)\end{array}$ & $\begin{array}{c}N_{\mathrm{Ca} \text { I }} \\
(4)\end{array}$ & $\begin{array}{c}\sigma_{\mathrm{A}, \mathrm{Ca} \mathrm{I}} \\
(5)\end{array}$ & $\begin{array}{c}{[\mathrm{Si} \mathrm{I} / \mathrm{Fe}]} \\
(6)\end{array}$ & $\begin{array}{c}\sigma_{\mathrm{Si} \text { I }} \\
(7)\end{array}$ & $\begin{array}{c}N_{\text {Si I }} \\
(8)\end{array}$ & $\begin{array}{c}\sigma_{\mathrm{A}, \mathrm{Si} \text { I }} \\
\quad(9)\end{array}$ & $\begin{array}{l}{\left[\mathrm{Ti} \mathrm{I} / \mathrm{Fe} \mathrm{I}_{\mathrm{I}}\right]} \\
\quad(10)\end{array}$ & $\begin{array}{l}\sigma_{\mathrm{Ti} \mathrm{I}} \\
(11)\end{array}$ & $\begin{array}{l}\mathrm{N}_{\mathrm{Ti} \text { I }} \\
(12)\end{array}$ & $\begin{array}{c}\sigma_{\mathrm{A}, \mathrm{TiI}} \\
(13)\end{array}$ & $\begin{array}{c}{[\mathrm{Ti} \mathrm{II} / \mathrm{Fe} \mathrm{II}]} \\
(14)\end{array}$ & $\begin{array}{l}\sigma_{\text {Ti II }} \\
(15)\end{array}$ & $\begin{array}{l}N_{\text {Ti II }} \\
(16)\end{array}$ & $\begin{array}{c}\sigma_{\mathrm{A}, \text { TiII }} \\
(17)\end{array}$ \\
\hline B006-G058 & 0.25 & 0.05 & 6 & 0.02 & 0.48 & 0.05 & 6 & 0.06 & 0.20 & 0.05 & 14 & 0.02 & 0.29 & 0.06 & 10 & 0.08 \\
\hline B012-G064 & 0.40 & 0.06 & 9 & 0.01 & 0.35 & 0.10 & 1 & 0.13 & $\ldots$ & $\ldots$ & $\ldots$ & $\ldots$ & 0.34 & 0.07 & 8 & 0.01 \\
\hline B029-G090 & 0.04 & 0.05 & 11 & 0.07 & 0.30 & 0.05 & 7 & 0.12 & 0.26 & 0.05 & 16 & 0.05 & 0.36 & 0.06 & 11 & 0.01 \\
\hline B034-G096 & 0.19 & 0.04 & 10 & 0.03 & 0.46 & 0.04 & 8 & 0.08 & 0.17 & 0.06 & 12 & 0.06 & 0.32 & 0.08 & 10 & 0.01 \\
\hline B045-G108 & 0.22 & 0.04 & 11 & 0.02 & 0.46 & 0.05 & 5 & 0.06 & 0.16 & 0.06 & 11 & 0.04 & 0.33 & 0.05 & 11 & 0.08 \\
\hline B048-G110 & 0.23 & 0.03 & 10 & 0.07 & 0.58 & 0.05 & 6 & 0.19 & 0.21 & 0.04 & 15 & 0.03 & 0.30 & 0.06 & 13 & 0.06 \\
\hline B088-G150 & 0.21 & 0.05 & 6 & 0.04 & $\ldots$ & $\ldots$ & $\ldots$ & $\ldots$ & 0.35 & 0.10 & 1 & 0.07 & 0.37 & 0.10 & 10 & 0.27 \\
\hline B110-G172 & 0.14 & 0.03 & 4 & 0.14 & 0.31 & 0.07 & 3 & 0.30 & 0.41 & 0.04 & 8 & 0.10 & 0.47 & 0.11 & 7 & 0.01 \\
\hline B163-G217 & 0.28 & 0.06 & 11 & 0.04 & 0.25 & 0.07 & 2 & 0.00 & 0.00 & 0.19 & 3 & 0.00 & 0.30 & 0.07 & 6 & 0.05 \\
\hline B171-G222 & 0.27 & 0.09 & 10 & 0.04 & 0.45 & 0.06 & 6 & 0.00 & -0.08 & 0.08 & 5 & 0.03 & 0.01 & 0.15 & 7 & 0.02 \\
\hline B182-G233 & 0.41 & 0.09 & 8 & 0.01 & 0.58 & 0.28 & 2 & 0.10 & 0.29 & 0.06 & 5 & 0.07 & 0.19 & 0.10 & 7 & 0.04 \\
\hline B193-G244 & 0.17 & 0.07 & 2 & 0.07 & 0.36 & 0.08 & 5 & 0.14 & 0.15 & 0.06 & 6 & 0.00 & 0.33 & 0.14 & 4 & 0.06 \\
\hline B225-G280 & 0.40 & 0.04 & 5 & 0.10 & 0.49 & 0.12 & 3 & 0.13 & $\ldots$ & $\ldots$ & $\ldots$ & $\ldots$ & 0.29 & 0.10 & 4 & 0.22 \\
\hline B232-G286 & 0.34 & 0.04 & 8 & 0.04 & $\ldots$ & $\ldots$ & $\ldots$ & $\ldots$ & 0.17 & 0.04 & 6 & 0.01 & 0.22 & 0.07 & 12 & 0.02 \\
\hline B235-G297 & 0.22 & 0.04 & 8 & 0.04 & 0.52 & 0.06 & 4 & 0.06 & 0.17 & 0.04 & 12 & 0.00 & 0.26 & 0.04 & 12 & 0.00 \\
\hline B240-G302 & 0.30 & 0.04 & 13 & 0.04 & 0.53 & 0.03 & 4 & 0.07 & 0.26 & 0.09 & 10 & 0.07 & 0.14 & 0.05 & 15 & 0.04 \\
\hline B311-G033 & 0.31 & 0.06 & 7 & 0.00 & 0.37 & 0.21 & 2 & 0.03 & 0.25 & 0.10 & 4 & 0.04 & 0.26 & 0.07 & 7 & 0.07 \\
\hline B312-G035 & 0.31 & 0.03 & 12 & 0.06 & 0.48 & 0.05 & 5 & 0.12 & 0.30 & 0.07 & 9 & 0.00 & 0.14 & 0.08 & 10 & 0.01 \\
\hline B358-G219 & 0.27 & 0.05 & 7 & 0.00 & $\ldots$ & $\ldots$ & $\ldots$ & $\ldots$ & 0.14 & 0.04 & 6 & 0.04 & 0.34 & 0.05 & 11 & 0.04 \\
\hline B381-G315 & 0.27 & 0.04 & 12 & 0.04 & 0.54 & 0.06 & 6 & 0.06 & 0.25 & 0.04 & 11 & 0.02 & 0.37 & 0.06 & 11 & 0.04 \\
\hline B383-G318 & 0.28 & 0.04 & 12 & 0.14 & 0.45 & 0.05 & 6 & 0.10 & 0.27 & 0.06 & 10 & 0.17 & 0.30 & 0.07 & 12 & 0.23 \\
\hline B386-G322 & 0.27 & 0.04 & 11 & 0.01 & 0.48 & 0.08 & 7 & 0.05 & 0.28 & 0.06 & 13 & 0.01 & 0.41 & 0.07 & 11 & 0.01 \\
\hline B384-G319 & 0.14 & 0.03 & 10 & 0.02 & 0.39 & 0.05 & 8 & 0.05 & 0.28 & 0.05 & 14 & 0.01 & 0.36 & 0.08 & 12 & 0.03 \\
\hline B403-G348 & 0.26 & 0.04 & 11 & 0.03 & 0.57 & 0.06 & 8 & 0.00 & 0.24 & 0.07 & 12 & 0.17 & 0.34 & 0.04 & 9 & 0.04 \\
\hline B405-G351 & 0.26 & 0.06 & 12 & 0.00 & 0.48 & 0.10 & 2 & 0.04 & 0.40 & 0.04 & 5 & 0.00 & 0.23 & 0.06 & 11 & 0.01 \\
\hline B457-G097 & 0.08 & 0.04 & 8 & 0.06 & 0.10 & 0.04 & 3 & 0.05 & 0.42 & 0.07 & 10 & 0.31 & 0.27 & 0.08 & 12 & 0.14 \\
\hline B514-MCGC4 & 0.45 & 0.04 & 9 & 0.05 & 0.66 & 0.10 & 1 & 0.09 & 0.06 & 0.04 & 7 & 0.07 & 0.17 & 0.06 & 15 & 0.06 \\
\hline G002 & -0.02 & 0.02 & 8 & 0.06 & 0.07 & 0.10 & 2 & 0.04 & -0.18 & 0.07 & 8 & 0.06 & -0.05 & 0.08 & 12 & 0.09 \\
\hline G327-MVI & 0.40 & 0.07 & 6 & 0.12 & 0.38 & 0.14 & 2 & 0.11 & 0.26 & 0.04 & 7 & 0.04 & 0.25 & 0.04 & 11 & 0.06 \\
\hline MCGC5-H10 & 0.18 & 0.04 & 9 & 0.02 & 0.37 & 0.12 & 4 & 0.03 & 0.05 & 0.09 & 7 & 0.01 & 0.25 & 0.05 & 14 & 0.01 \\
\hline MGC1 & 0.18 & 0.04 & 7 & 0.00 & 0.53 & 0.10 & 2 & 0.04 & 0.06 & 0.02 & 6 & 0.01 & 0.14 & 0.05 & 14 & 0.01 \\
\hline
\end{tabular}

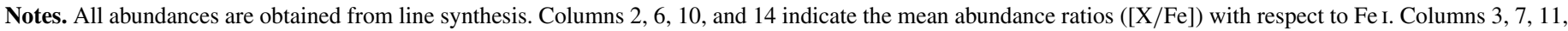

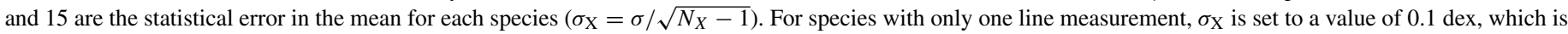

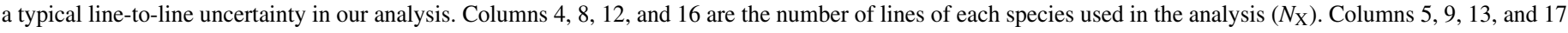
are the uncertainty in the abundance due to the age of the $\mathrm{CMD}\left(\sigma_{\mathrm{A}, \mathrm{X}}\right)$.

strong and isolated enough for clean measurement in most GCs. The $6169 \AA$ line is a blend of two Ca I features and is only included when the abundance can be cleanly measured. The $6455 \AA, 6464 \AA$, and $6508 \AA$ lines are weak and usually only measured in the more metal-rich GCs that have low velocity dispersions.

The Si I line list includes 11 lines, which are all weaker features than most of the Ca I lines. The $5793 \AA$, $5948 \AA$, $6721 \AA$, and $7932 \AA$ are the weakest and are only measured in a handful of GCs that are metal rich and have low velocity dispersions. The $7415 \AA$ and $7423 \AA$ are blended with Ni I lines, and were only measured when the velocity dispersion was low enough that the line profiles were sufficiently separated.

Our Ti I line list includes 22 lines, most of which are blended with other features to some extent. We include measurements of the blended lines when the line profiles are sufficiently separated that we are confident with the abundance measurement. Figure 18 shows a typical case where the line is blended with a nearby feature but the abundance is well determined. The Ti I lines that are most isolated are $4991 \AA$, $5866 \AA$, and $6743 \AA$. The redder Ti I lines $(\lambda>5200 \AA)$ are generally weaker and so are not usually cleanly measured in more metal-poor GCs. The $5173 \AA$ and $5192 \AA$ are in the $\mathrm{Mg}$ b region and are usually measured in more metal-poor GCs where the continuum is well determined. Likewise, the $6554 \AA$ and $6556 \AA$ Ti I lines are near $\mathrm{H} \alpha$ and are only included when the wings of $\mathrm{H} \alpha$ are not significant, so that the continuum is well determined.

17 lines are included in the Ti II line list; most are somewhat blended, similar to Ti I. The most isolated Ti II lines are $4501 \AA$, $4589 \AA$, and $5381 \AA$. The $5185 \AA$ line is in the $\mathrm{Mg}$ b region, and was only included when the continuum was well determined. The $4865 \AA$ line is near $\mathrm{H} \beta$ and was only included when the wings of $\mathrm{H} \beta$ did not affect the continuum near the line.

For consistency, we have re-measured the abundances of the $\alpha$ elements with IL synthesis for the GCs presented in C09. In Figure 19, we compare the results for the EW analysis in C09 to the results measured using line synthesis in this work. In general, the results are in good agreement, and we find that when using synthesis the number of lines recoverable is generally larger, and the statistical error is reduced. Only Ti II abundances appear to be significantly different, which may be because most of the Ti II lines suffer from line blending. This highlights the better precision obtained using line synthesis, which is especially important for elements that have only a handful of analyzable spectral features, such as Si I. We also note that we were able to add measurements for Si I for B405 and $\mathrm{Ti}$ I for B358, which we were not able to measure with EWs in $\mathrm{C} 09$. 

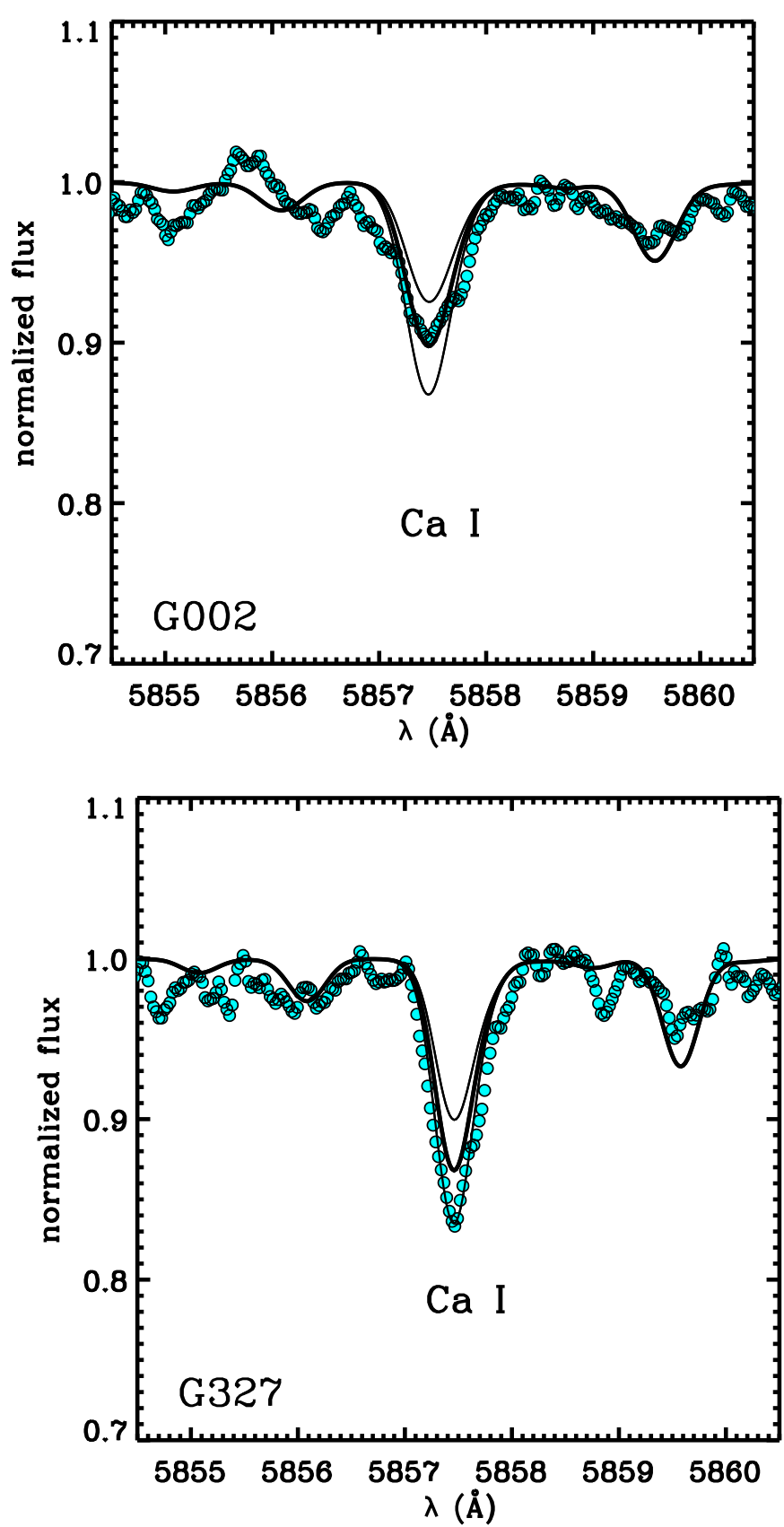

Figure 17. Example Ca I synthesis fits for G002 (top) and G327 (bottom), which have similar metallicities. Points show the data, which have been smoothed by 5 pixels for presentation. Solid lines correspond to ratios of $[\mathrm{Ca} / \mathrm{Fe}]=$ $-0.3,+0.0,+0.30$. The best fitting abundance for $\mathrm{G} 002$ is $[\mathrm{Ca} / \mathrm{Fe}]=+0.0$, while for $\mathrm{G} 327$ is $[\mathrm{Ca} / \mathrm{Fe}]=+0.3$.

(A color version of this figure is available in the online journal.)

The results in Tables 8 and 9 show that the total uncertainty in the abundance ratios, which is determined by adding the $\sigma_{\mathrm{X}}$ and $\sigma_{\mathrm{A}, \mathrm{X}}$ in quadrature, is usually $<0.1 \mathrm{dex}$, and the age uncertainty, $\sigma_{\mathrm{A}, \mathrm{X}}$, is usually less than or comparable to the statistical uncertainty, $\sigma_{\mathrm{X}}$. However, in some cases, the $\sigma_{\mathrm{A}, \mathrm{X}}$ dominates the abundance ratio uncertainty. It is expected that this would be the case for GCs with larger age uncertainties, like B193 and B457, which have uncertainties of 5 and 4 Gyr, respectively. However, these GCs also have the lowest $\mathrm{S} / \mathrm{N}$ data of the current sample. If the age uncertainty was solely due to a stellar population mismatch in the true and theoretical CMDs, this uncertainty would remain even if better quality, higher
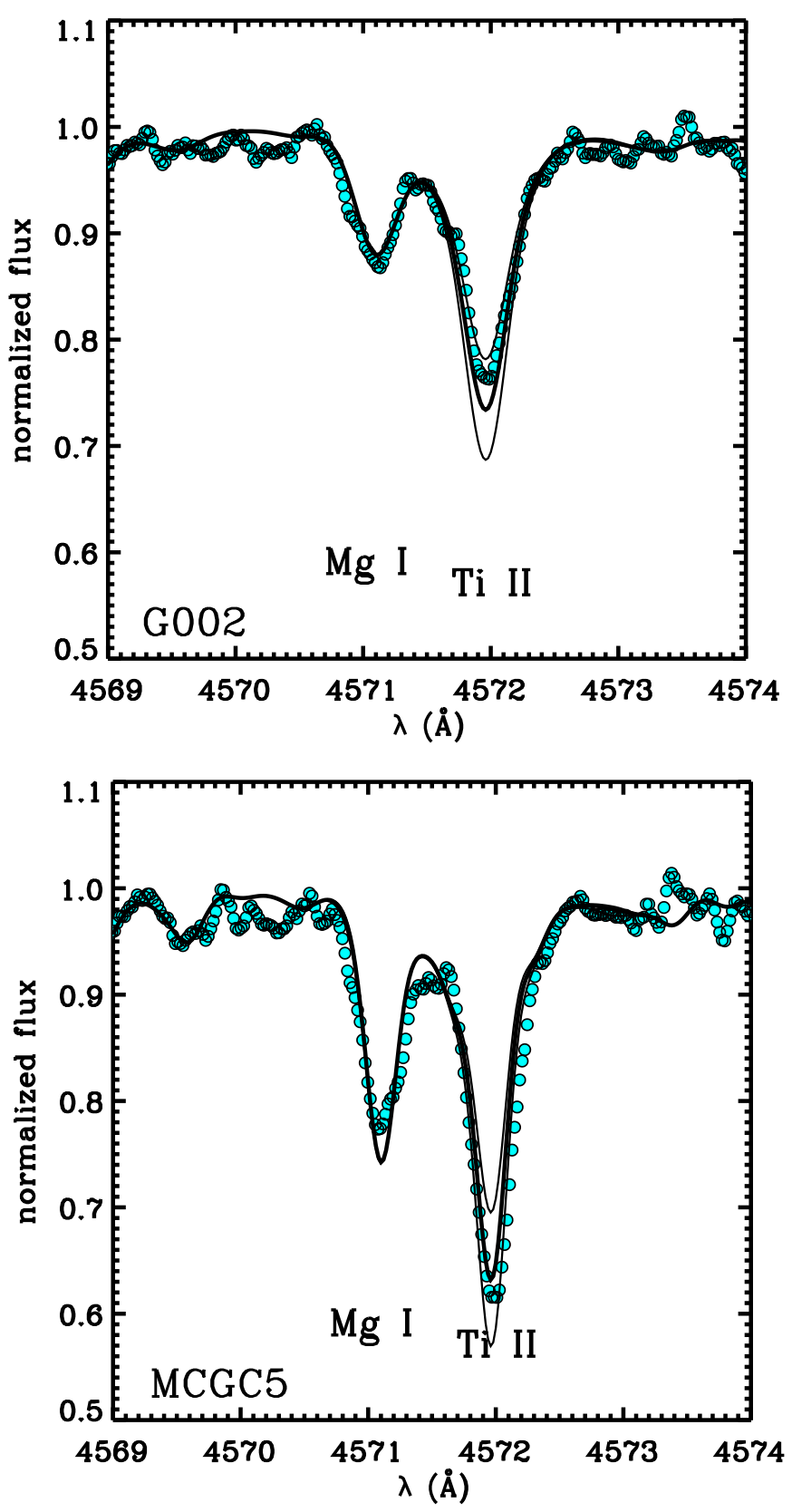

Figure 18. Example Ti II synthesis fits for G002 (top) and MCGC5 (bottom). Points show the data, which have been smoothed by 5 pixels for presentation. Solid lines correspond to ratios of $[\mathrm{Ti} / \mathrm{Fe}]=-0.5,+0.0,+0.50$, and the mean $[\mathrm{Mg} / \mathrm{Fe}]$ for each $\mathrm{GC}([\mathrm{Mg} / \mathrm{Fe}]=-0.5$ for $\mathrm{G} 002$ and $[\mathrm{Mg} / \mathrm{Fe}]=-0.1$ for MCGC5) has been adopted to account for blending with the $\mathrm{Mg}$ I feature. G002 has a lower [Ti/Fe] than MCGC5.

(A color version of this figure is available in the online journal.)

$\mathrm{S} / \mathrm{N}$ data were obtained. However, since we determine the age using the behavior of the abundances measured from different Fe I lines, it is possible that reducing the line to line scatter with better quality data could also have an impact on the age determination.

Because we have a large sample of both Ti I and Ti II abundances, it is interesting to compare the results for neutral and ionized species, as we did for Fe I and Fe II in Section 4.2. This comparison is shown for the absolute $\mathrm{Ti}$ I and $\mathrm{Ti}$ II abundances in Figure 20. We find that the abundances we obtain for Ti I are consistent with those of Ti II, just as the Fe I abundances were consistent with the Fe II abundances. 


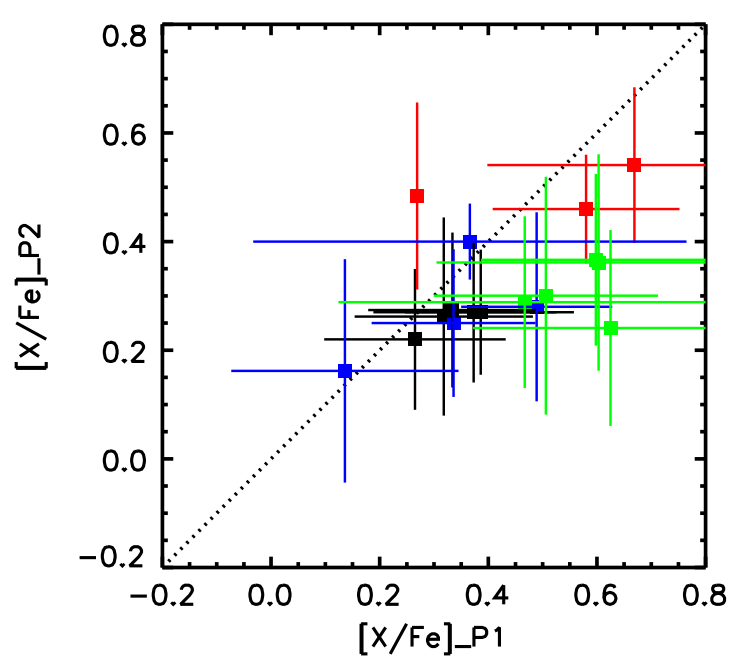

Figure 19. Comparison of $\alpha$ element ratios measured using EWs in C09 ([X/Fe]_P1) to those measured using spectral synthesis in this work ([X/ Fe]_P2). Black, red, blue and green show CaI, Si I, Ti I and Ti II ratios, respectively. The dotted line shows 1:1 correspondence.

(A color version of this figure is available in the online journal.)

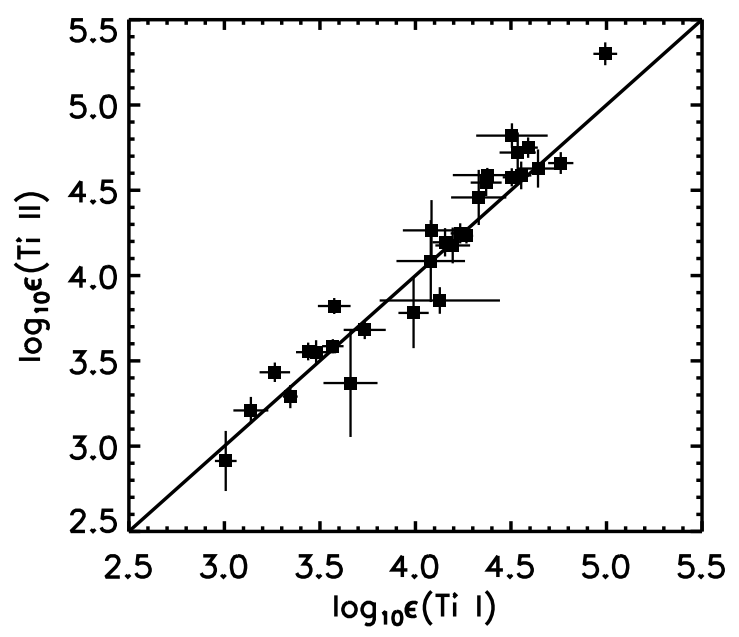

Figure 20. Same as Figure 16 for Ti I and Ti II abundances.

In Figure 21, we compare the $\alpha$ element ratios as a function of $[\mathrm{Fe} / \mathrm{H}]$ for M31 and the MW. For the MW comparison we use the compilation of GC mean abundances in Pritzl et al. (2005), which were obtained from homogenizing the abundances of individual stars by different authors. In general, the $[\mathrm{Ca} / \mathrm{Fe}]$, [Si/Fe], [Ti I/Fe], and [Ti II/Fe] abundance patterns of the M31 GCs are similar to the MW abundance patterns. It is interesting to compare the $[\alpha / \mathrm{Fe}]$ plateau values because the plateau is in principle sensitive to the relative numbers of high mass stars, and contains information about the early halo stellar population history. We calculate the mean plateau values using all GCs with $[\mathrm{Fe} / \mathrm{H}]<-0.7$; for easy comparison, the mean values for the M31 sample and the stellar GC data from Pritzl et al. (2005) are listed in Table 10. We find that the mean plateau values for the four individual elements, as well as a mean of the sample as a whole, agree very well. In addition, we show the mean obtained from the four elements for each individual GC in both samples in Figure 22, where the agreement for the M31 GCs and MW GCs in the plateau region is clear. Interestingly, we also note that the $[\mathrm{Ca} / \mathrm{Fe}]$ abundances in Figure 21 also follow the MW field star abundances very closely in the "knee" region from $[\mathrm{Fe} / \mathrm{H}] \sim-1$ to $[\mathrm{Fe} / \mathrm{H}] \sim 0$, perhaps more closely than
Table 10

Mean $\alpha$-Element Abundance Comparison

\begin{tabular}{lcc}
\hline \hline & $\begin{array}{c}\text { M31 ILS } \\
{[\mathrm{Fe} / \mathrm{H}]<-0.7}\end{array}$ & $\begin{array}{c}\text { MW, GC Stars } \\
{[\mathrm{Fe} / \mathrm{H}]<-0.7}\end{array}$ \\
\hline$[\mathrm{Ca} / \mathrm{Fe}]$ & $+0.26 \pm 0.10$ & $+0.27 \pm 0.13$ \\
{$[\mathrm{Si} / \mathrm{Fe}]$} & $+0.45 \pm 0.14$ & $+0.38 \pm 0.18$ \\
{$[\mathrm{TiI} / \mathrm{Fe}]$} & $+0.21 \pm 0.13$ & $+0.23 \pm 0.16$ \\
{$[\mathrm{TiII} / \mathrm{Fe}]$} & $+0.26 \pm 0.10$ & $+0.27 \pm 0.18$ \\
\hline$[$ Alpha/Fe $]$ & $+0.28 \pm 0.16$ & $+0.29 \pm 0.17$
\end{tabular}

Note. ${ }^{a}$ Using mean GC abundances from Pritzl et al. (2005).

the MW's own GCs. The decrease in $[\mathrm{Ca} / \mathrm{Fe}]$ in the knee region may be interpreted as an increasingly dominant contribution of SN Ia enrichment over SN II enrichment in the ISM, since SN Ia produce $\mathrm{Fe}$ in larger amounts than $\mathrm{SN}$ II.

One $\mathrm{GC}, \mathrm{G} 002$, has $\alpha$-element abundance ratios that are lower than those of the other GCs at similar metallicity ([Fe/ $\mathrm{H}] \sim-1.6)$. We have highlighted this GC with a different symbol in Figure 21 for clarity. The difference in $\mathrm{Ca}$ I line strength between G002 and another GC with similar metallicity and velocity dispersion, G327, can be seen in Figure 17, which also visually demonstrates the accuracy of the synthesis fits. We also find other GCs, such as B457 and B171, that have two species with $[\alpha / \mathrm{Fe}]$ that are lower than the average at their respective metallicities. The two GCs also have slightly different $\alpha$ element behaviors from each other. B457 only has lower than average $[\mathrm{Ca} / \mathrm{Fe}]$ and $[\mathrm{Si} / \mathrm{Fe}]$, while $\mathrm{B} 171$ has lower than average [Ti I/Fe] and [Ti II/Fe]. In general, lower $\alpha$ element abundance ratios can potentially indicate late-time accretion from dwarf galaxies. For example, similar deficits in $[\alpha / \mathrm{Fe}]$ are found in the MW GC Ruprecht 106 (Villanova et al. 2013), which is thought to have formed outside the MW. Lower $[\alpha / \mathrm{Fe}]$ abundances are also seen in Sagittarius GCs that are recently or currently being accreted into the MW GC system (e.g., Forbes \& Bridges 2010; Sbordone et al. 2005; Cohen 2004), although these GCs have higher $[\mathrm{Fe} / \mathrm{H}]$ than $\mathrm{G002}$. We discuss insights into the accretion history of M31 in more detail in Section 5.3.

Figure 21 also shows that, as in the MW, in M31 the different $\alpha$ elements show subtle differences in their abundance patterns. For example, $[\mathrm{Si} / \mathrm{Fe}]$ ratios are typically higher than $[\mathrm{Ca} /$ $\mathrm{Fe}]$ or $[\mathrm{Ti} / \mathrm{Fe}]$ ratios, which is a reflection of their different formation channels. This is important to keep in mind, because in extragalactic studies a "mean" $[\alpha / \mathrm{Fe}]$ ratio is often the only measurement that can be obtained, but as Figure 21 demonstrates this can potentially wash out the interesting and constraining behavior of these elements. In Figure 22, we average the abundances we obtain for $[\mathrm{Ca} / \mathrm{Fe}],[\mathrm{Si} / \mathrm{Fe}],[\mathrm{Ti} / \mathrm{Fe}]$, and [Ti II/Fe] to show how some information is lost when examining a mean $[\alpha / \mathrm{Fe}]$ alone, although we note that one would come to the same the general conclusion that the abundances in M31 are similar to the MW. In terms of details that are lost, Figure 22 shows that the mean $[\alpha / \mathrm{Fe}]$ for B457 and B171 now look more similar to the bulk of the GCs, even though half of their $[\alpha / \mathrm{Fe}]$ ratios are low. In the mean, G002 is the only GC that clearly has a different $[\alpha / \mathrm{Fe}]$ abundance pattern. We also find that the highest metallicity cluster, $\mathrm{B} 193$, is at higher mean $[\alpha / \mathrm{Fe}]$ than the MW disk stars, but if we separate the individual elements we find that B193 only appears to be particularly inconsistent in $[\mathrm{Si} / \mathrm{Fe}]$ and $[\mathrm{Ti} \mathrm{II} / \mathrm{Fe}]$. Together, this shows that the mean $[\alpha / \mathrm{Fe}]$ is certainly a useful tool for learning about the overall chemical evolution in other galaxies, but the extra information 

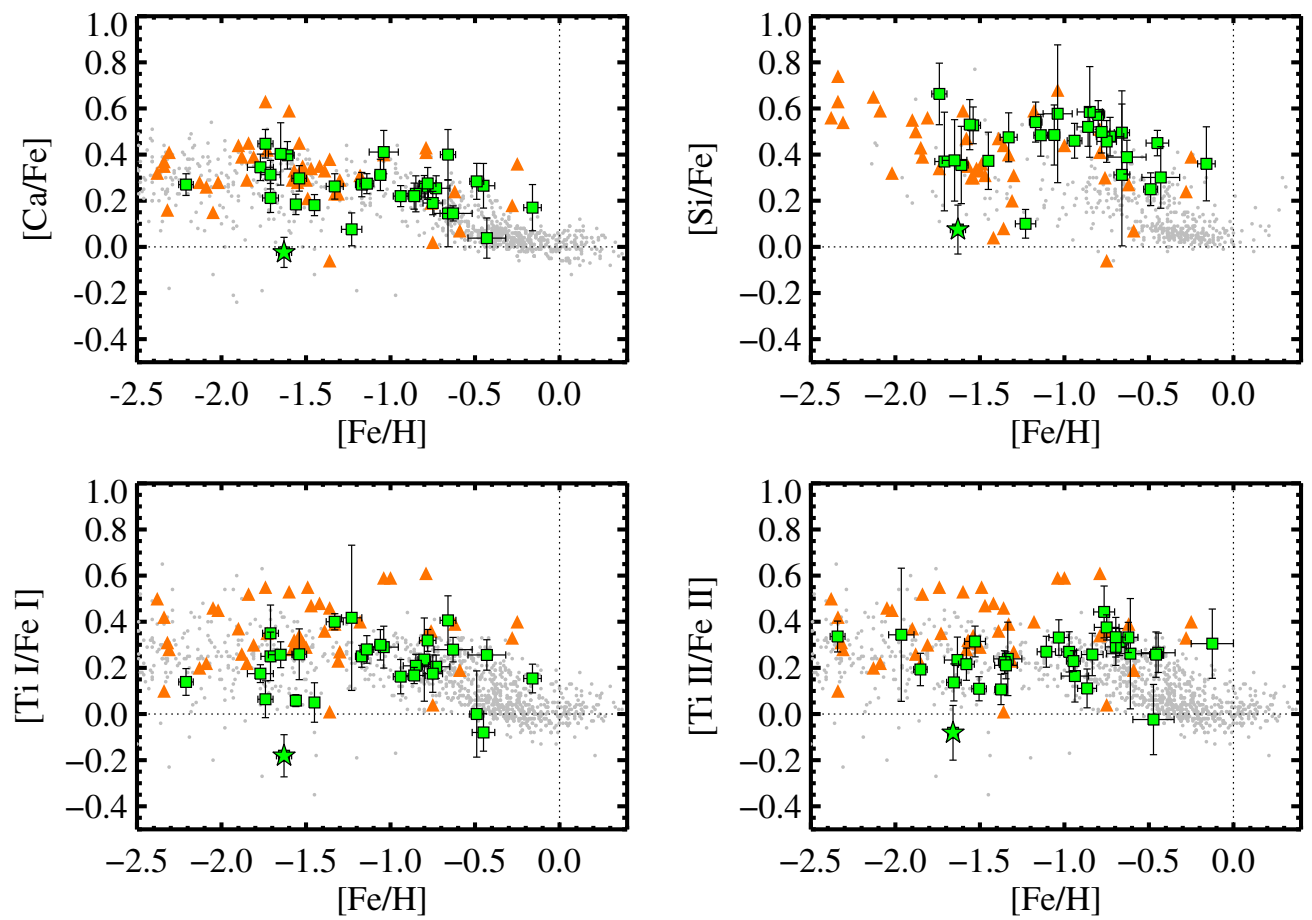

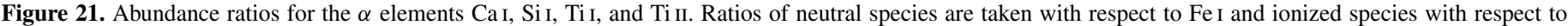

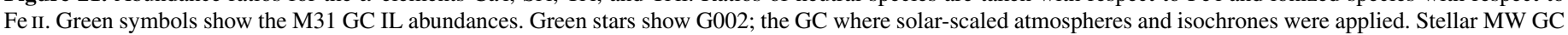

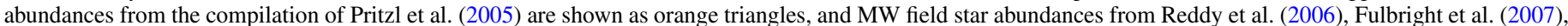
Barklem et al. (2005), and the compilation of Venn et al. (2004) are shown as gray circles.

(A color version of this figure is available in the online journal.)

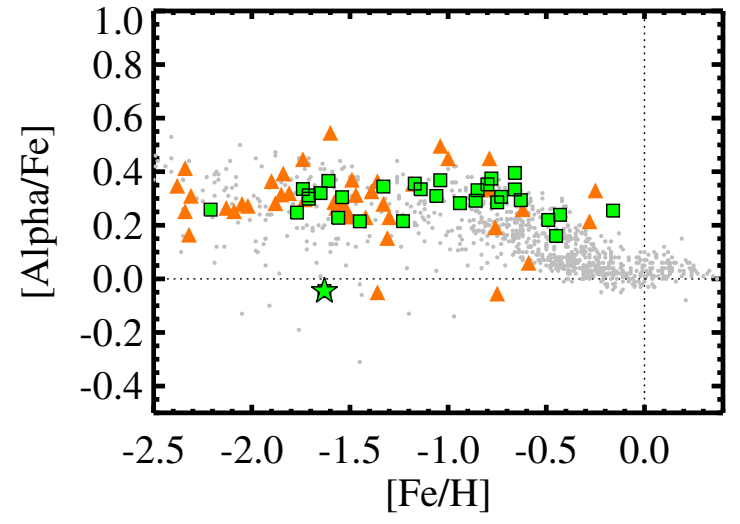

Figure 22. Mean $\alpha$ abundances from Ca I, Si I, Ti I, and Ti II ratios. Symbols and references are the same as in Figure 21.

(A color version of this figure is available in the online journal.)

from having precise measurements of several $\alpha$ elements shows promise for new insights in the details of galaxy evolution.

In summary, we measure Ca I, Si I, Ti I, and Ti II abundances in the majority of the GCs in our current M31 sample, providing a novel look at the evolution of $[\alpha / \mathrm{Fe}]$ over a wide range of metallicity in this galaxy. We find that the M31 GCs generally have properties similar to MW GCs and disk stars, and discuss further implications on the star formation history of M31 in Section 5.

\subsection{Light Elements}

We measure light element abundances for $\mathrm{Mg}$ I, $\mathrm{NaI}$, and Al I, which are presented in Table 8 . Like the $\alpha$ elements, these abundances were measured with IL spectral synthesis. The abundance ratios for these elements as a function of $[\mathrm{Fe} / \mathrm{H}]$ are shown in Figure 23. These light elements are interesting because they are not necessarily monometallic in GCs, unlike $[\mathrm{Fe} / \mathrm{H}]$ and the $\alpha$ elements discussed in Section 4.3. Instead, they have been observed to vary star-to-star in patterns that reflect high temperature proton capture nucleosynthesis (see review by Gratton et al. 2004 and references therein). When these patterns are present $[\mathrm{Na} / \mathrm{Fe}]$ and $[\mathrm{Al} / \mathrm{Fe}]$ are enhanced above the normal values of $\mathrm{MW}$ field stars, and $[\mathrm{O} / \mathrm{Fe}]$ and $[\mathrm{Mg} / \mathrm{Fe}]$ are depleted with respect to normal values, which has led these patterns to be dubbed the " $\mathrm{Na}-\mathrm{O}$ " and " $\mathrm{Mg}-\mathrm{Al}$ anticorrelations." The range of the star-to-star abundance variations is different between MW GCs and is thought to be related to the mechanism that causes this behavior in GCs, although a consensus on a complete theoretical understanding has yet to be found (e.g., Carretta 2006; Decressin et al. 2009; Carretta et al. 2010b; D'Ercole et al. 2012; Conroy 2012; Bastian et al. 2013; Maxwell et al. 2014). The full extent of the Na-O anti correlation is observed to depend on present day GC mass, and there observationally appears to be a minimum GC mass for correlations to be present (e.g., Carretta et al. 2010b). Variations in the light elements have been directly observed in GC stellar samples in both the MW and external galaxies such as the LMC (e.g., Mucciarelli et al. 2009). They have also been indirectly observed in the high-resolution IL of GCs in M31 in C09 and Colucci et al. (2013a), the LMC (C12), Fornax (Larsen et al. 2012) and in MW GCs in Sakari et al. (2013). The effect of the variations on the abundances obtained from IL spectra is thought to be a bias in the IL abundance toward higher ratios in $[\mathrm{Na} / \mathrm{Fe}]$ and $[\mathrm{Al} / \mathrm{Fe}]$, and toward lower values in $[\mathrm{Mg} / \mathrm{Fe}]$. The abundance variations are also believed to bias measurements of $[\mathrm{Mg} / \mathrm{Fe}]$ in low-resolution GC IL 

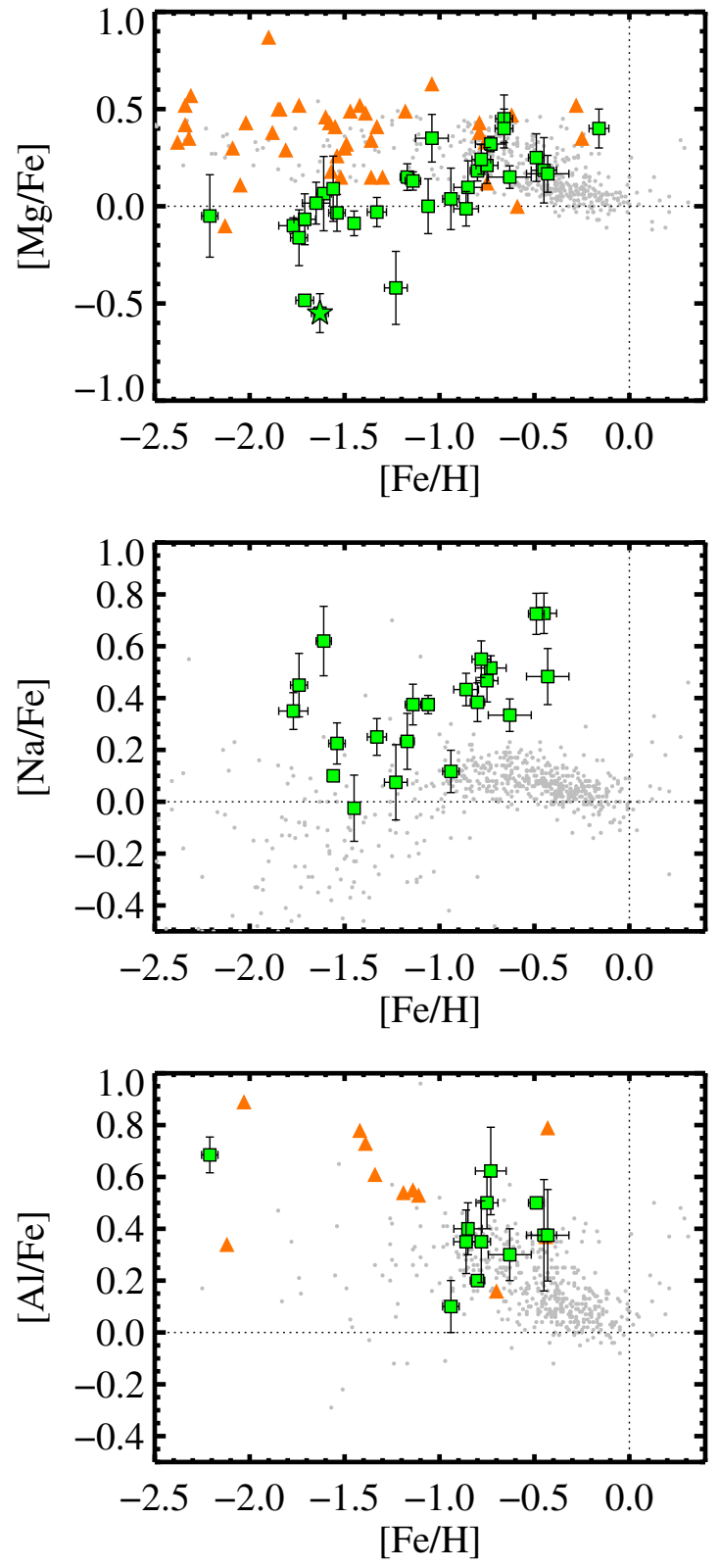

Figure 23. Light element abundance ratios for $\mathrm{Mg}$ I, Na I, and Al I. Symbols and references are the same as in Figure 21, with the addition of $\mathrm{Na}$ and $\mathrm{Al}$ stellar abundances from Fulbright (2000), Bai et al. (2004), and Carretta (2006).

(A color version of this figure is available in the online journal.)

spectra, as shown in Schiavon et al. (2013) for a large sample of M31 GCs.

Figure 23 shows further indirect evidence for star-to-star abundance variations in M31 GCs. First, we find $[\mathrm{Mg} / \mathrm{Fe}]$ abundances that are lower on average than the field star plateau values, particularly at $[\mathrm{Fe} / \mathrm{H}]<-0.7$. Our $\mathrm{Mg}$ I measurements are obtained from a handful of weak lines across the full wavelength coverage of HIRES (4167 $\AA, 4571 \AA, 4703 \AA$, $5528 \AA, 5711 \AA, 7387 \AA$ ) in order to explicitly avoid the strongest $\mathrm{Mg} \mathrm{I}$ lines in the $\mathrm{Mg}$ b region so as to limit nonLTE or saturation problems. The fact that the more metalpoor GCs are particularly biased to low $[\mathrm{Mg} / \mathrm{Fe}]$ abundances is very interesting, although not entirely understood. We note that Larsen et al. (2012) also find particularly low values of $[\mathrm{Mg} / \mathrm{Fe}]$ in very metal-poor Fornax GCs, which is consistent with the picture we find here in $\mathrm{M} 31$. The lowest $[\mathrm{Mg} / \mathrm{Fe}]$ we
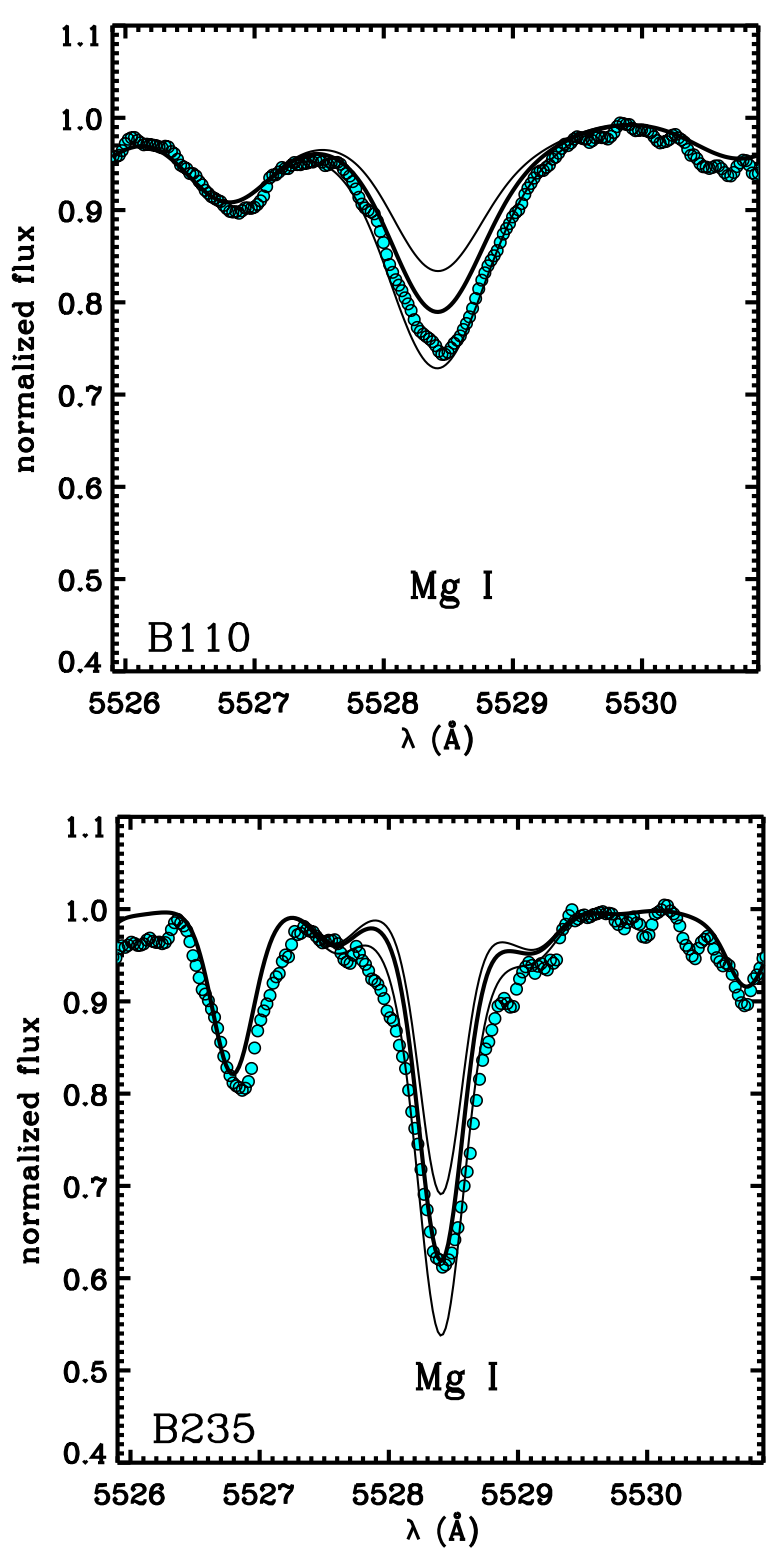

Figure 24. Example $\mathrm{Mg}_{\mathrm{I}}$ synthesis fits for B110 (top) and B235 (bottom). Points show the data, which have been smoothed by 5 pixels for presentation. Solid lines correspond to ratios of $[\mathrm{Mg} / \mathrm{Fe}]=-0.5,+0.0,+0.5$. The two GCs have similar metallicities of $[\mathrm{Fe} / \mathrm{H}] \sim-0.7$ but different velocity dispersions; $\mathrm{B} 235$ has a lower $[\mathrm{Mg} / \mathrm{Fe}]$ than $\mathrm{B} 110$.

(A color version of this figure is available in the online journal.)

find is for $\mathrm{G} 002$, of $[\mathrm{Mg} / \mathrm{Fe}]=-0.5$, and an example of a clean measurement with that abundance for the $4571 \AA$ line is shown in Figure 18. We show two examples of the Mg I $5528 \AA$ line in Figure 24 for GCs with higher metallicities of $[\mathrm{Fe} / \mathrm{H}] \sim-0.7$. B110 has a $[\mathrm{Mg} / \mathrm{Fe}] \sim+0.4$, which is similar to the other $\alpha$ element plateau values, but B235 clearly has a lower ratio of $[\mathrm{Mg} / \mathrm{Fe}] \sim 0.0$. We note that $[\mathrm{Mg} / \mathrm{Fe}]$ abundances as low as these have also been measured in a subset of stars in the MW outer halo GC NGC 2419 by Cohen \& Kirby (2012).

We also find elevated $[\mathrm{Na} / \mathrm{Fe}]$, which can be seen at all $[\mathrm{Fe} / \mathrm{H}]$ in Figure 23. The $\mathrm{Na} \mathrm{I}$ abundances are measured from the unsaturated $5582 \AA$ and $6160 \AA$ doublets, which minimizes non-LTE effects. Although we only have four transitions to work with, the Na I lines are moderately strong and the $5582 \AA$ doublet in particular is only partially blended, so we are able to make Na I measurements for most of the range in $[\mathrm{Fe} / \mathrm{H}]$ of the 


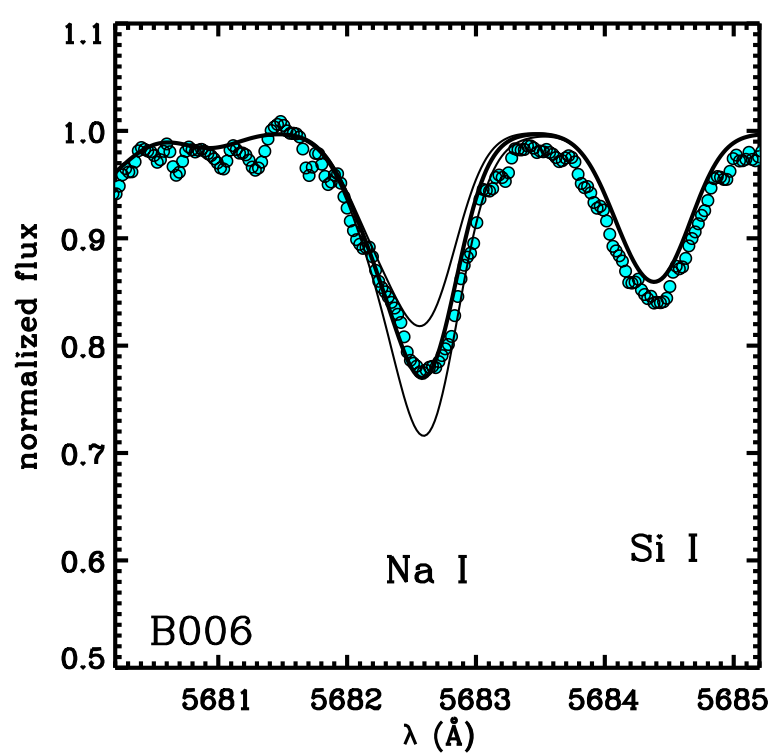

Figure 25. Example Na I synthesis fits for B006. Points show the data, which have been smoothed by 5 pixels for presentation. Solid lines correspond to ratios of $[\mathrm{Na} / \mathrm{Fe}]=+0.0,+0.5,+1.0$. The mean $[\mathrm{Si} / \mathrm{Fe}]=+0.48$ has been adapted to account for blends.

(A color version of this figure is available in the online journal.)

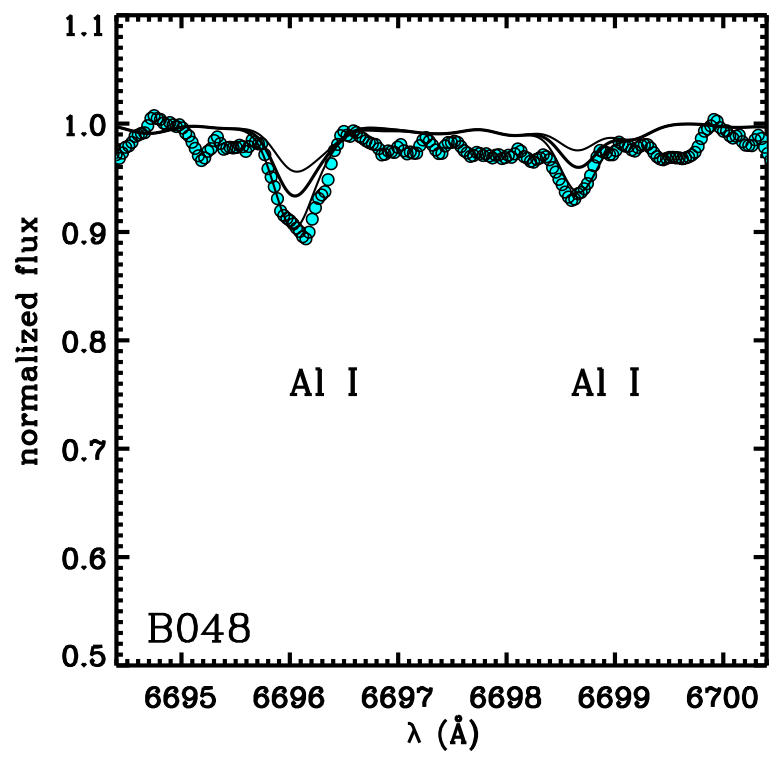

Figure 26. Example Al I synthesis fits for B048. Points show the data, which have been smoothed by 5 pixels for presentation. Solid lines correspond to ratios of $[\mathrm{Al} / \mathrm{Fe}]=-0.3,+0.0,+0.3$.

(A color version of this figure is available in the online journal.)

sample. An example of an enhanced $[\mathrm{Na} / \mathrm{Fe}]$ measurement is shown in Figure 25 for B006, which has a mean of $[\mathrm{Na} / \mathrm{Fe}]=$ +0.52 .

The $[\mathrm{Al} / \mathrm{Fe}]$ abundances that we measure are also significantly super-solar; however, they are primarily measured at higher metallicities and have more overlap with abundances in normal MW field stars. With the exception of the metal-poor GC B358 in C09, the Al I abundances are entirely measured from the $6696 \AA$ doublet, which should not require substantial non-LTE corrections. The Al I measurements are from some of the weakest lines that we are able to cleanly measure. An example is shown in Figure 26 for B048, where we also show
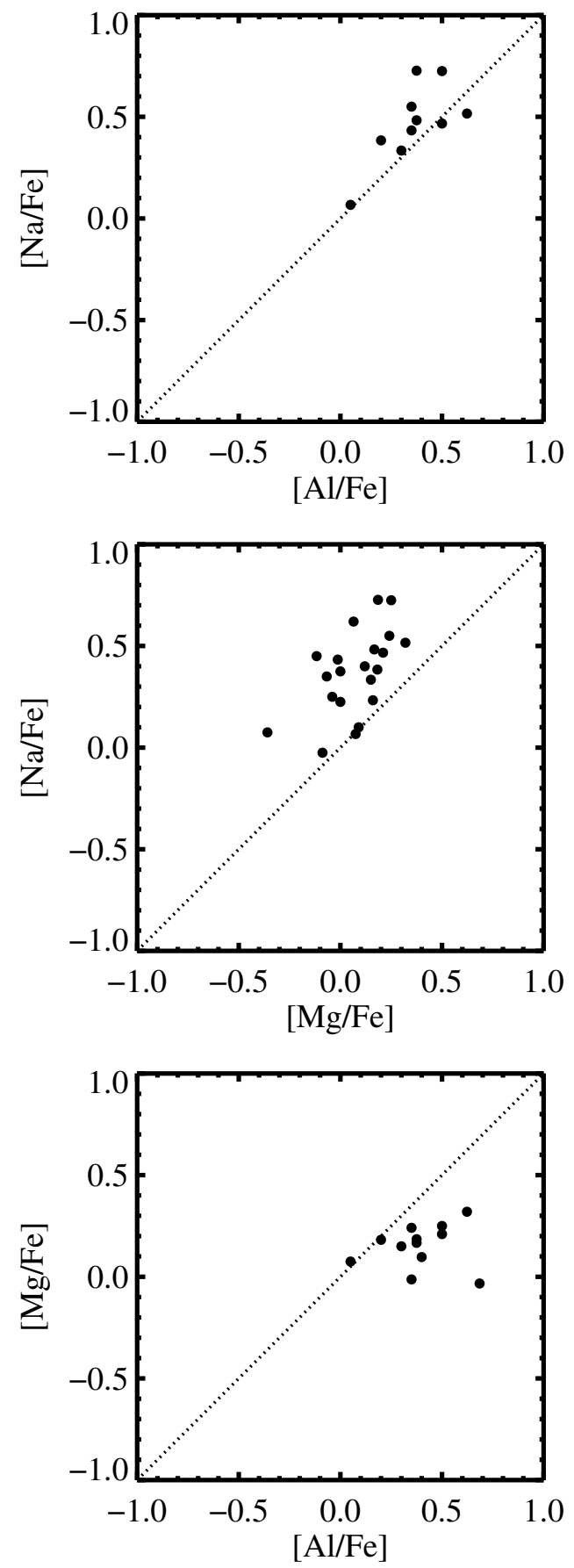

Figure 27. Possible correlations in the light element abundance ratios in M31 GCs. The dotted line shows a 1:1 correspondence. We find initial evidence that $[\mathrm{Na} / \mathrm{Fe}]$ may be loosely correlated with $[\mathrm{Al} / \mathrm{Fe}]$ and $[\mathrm{Mg} / \mathrm{Fe}]$, but $[\mathrm{Al} / \mathrm{Fe}]$ does not appear correlated with $[\mathrm{Mg} / \mathrm{Fe}]$.

the subtle differences between syntheses with $[\mathrm{Al} / \mathrm{Fe}]=-0.3$, +0.0 , and +0.3 . B048 has an $[\mathrm{Fe} / \mathrm{H}]=-0.89$, which is approximately the lowest metallicity for which we are able to cleanly measure an $\mathrm{Al}$ I abundance from these weak features, which are also not usually possible to measure for GCs with large velocity dispersions.

In Figure 27, we compare the behaviors of the light element abundance ratios with each other in order to look for possible correlations in the measurements. It is important to remember, however, that the comparison of IL flux weighted average values of the light element ratios are fundamentally different 
comparisons from the standard $\mathrm{Na}-\mathrm{O}, \mathrm{Mg}-\mathrm{Al}$ anti-correlations of individual stars within a GC. We find evidence that $[\mathrm{Na} / \mathrm{Fe}]$ is loosely correlated with $[\mathrm{Al} / \mathrm{Fe}]$ and $[\mathrm{Mg} / \mathrm{Fe}]$, but $[\mathrm{Mg} / \mathrm{Fe}]$ and $[\mathrm{Al} / \mathrm{Fe}]$ do not appear correlated. Note that not all GCs have measurements of all three elements, and $\mathrm{Al} I$ is only measured in the highest metallicity GCs, which may contribute to the lack of a correlation with $[\mathrm{Mg} / \mathrm{Fe}]$. Naively, we may conclude that the possibility of correlations between some of these elements simply shows that there is some consistency to whatever mechanism is driving the star-to-star abundance variations, although it is unclear why $[\mathrm{Mg} / \mathrm{Fe}]$ and $[\mathrm{Al} / \mathrm{Fe}]$ would not be correlated if their relative amounts are only determined by high temperature $p$ capture reaction chains. Indeed, Carretta (2006) also find that stars within MW GCs do not always show a clearly defined $\mathrm{Mg}-\mathrm{Al}$ relationship, and that large changes in $\mathrm{Al}$ are only accompanied by small changes in $\mathrm{Mg}$, which is consistent with what we find here in the IL abundances.

In summary, all three of the light elements we measure, $\mathrm{Mg}$ I, $\mathrm{NaI}$, and $\mathrm{AlI}$, appear to confirm the widespread existence of star-to-star abundance variations in M31.

\section{DISCUSSION}

In the following sections, we first examine possible relationships of the M31 GC abundances with other GC properties, such as luminosity, mass and velocity dispersion. We then discuss the abundances with respect to properties of the host galaxy, M31, such as radius. Next, we discuss implications that our measurements have for the star formation and accretion history of M31. Finally, we present comparisons of the high-resolution measurements with previous studies using other techniques.

\subsection{Behavior with GC Properties}

While our sample is smaller than those in photometric or low-resolution spectra studies of M31 GCs, and obviously not complete, it is interesting to look for relationships of the high precision abundances with other GC properties. Relationships of GC luminosity with $[\mathrm{Fe} / \mathrm{H}]$, or mass-metallicity relationships, have been extensively searched for in GC systems in other galaxies (e.g., Harris et al. 2006; Strader \& Smith 2008; Mieske et al. 2010). While correlations with mass and metallicity have not been found in the MW (Strader \& Smith 2008 with data from Harris 1996) or M31 GC systems previously (e.g., Barmby et al. 2000; Caldwell et al. 2011), they are commonly found in the metal-poor component of GC systems in earlytype galaxies (Harris 2009). The low-metallicity GC massmetallicity relationship, or "blue tilt," is thought to be created by GC "self-enrichment" (e.g., Bailin \& Harris 2009; Mieske et al. 2010; Goudfrooij \& Kruijssen 2014), where massive GCs are able to retain a significant fraction of their early SN II ejecta in order to incorporate it into their late-forming stars. It is not yet clear how the mass-metallicity relationship of GCs seen in early type galaxies may be related to the multiple generations of stars seen in MW and LMC GCs (e.g., Piotto 2009; Milone et al. 2009), or the possible mechanism for star-to-star light element variations.

In Figure 28, we show our $[\mathrm{Fe} / \mathrm{H}]$ measurements as a function of reddening-corrected absolute magnitude in $V$, and we have visually separated "metal-poor" and "metal-rich" subpopulations with blue and red symbols, respectively. We use a value of $[\mathrm{Fe} / \mathrm{H}]=-1.2$ to divide the sample, which is approximately the location of the minimum between peaks in the MW's [Fe/H] distribution (Harris 1996, 2010 revision).

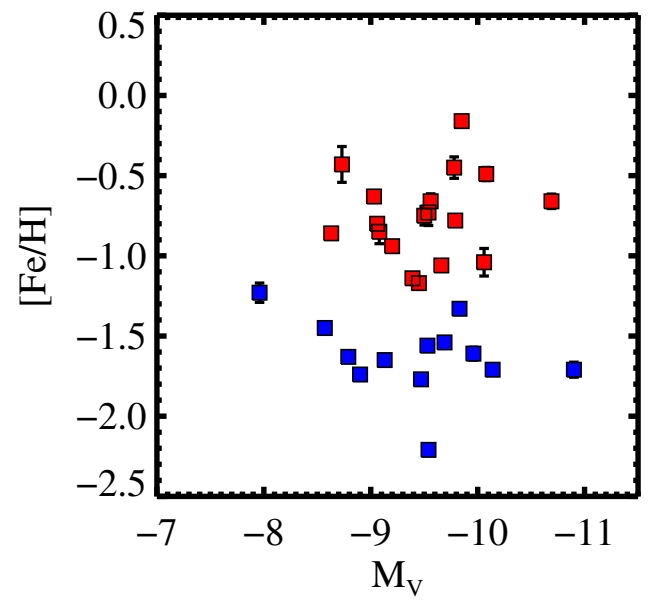

Figure 28. Luminosity-metallicity relationship for the M31 GC sample. We divide the populations at $[\mathrm{Fe} / \mathrm{H}]=-1.2$. Magnitudes are listed in Table 1, and are taken from Galleti et al. (2004), $E(B-V)$ and associated references are found in Table 1, and we assume a distance modulus of 24.47 (McConnachie et al. 2005) and extinction parameter $R_{V}=3.1$.

(A color version of this figure is available in the online journal.)

Although our GC sample is small compared to the total GC population in M31, it is nevertheless interesting to separate behaviors of the metal-poor and metal-rich subpopulations because of the long history of study of GC subpopulations in general (e.g., Brodie \& Strader 2006). Approximately half of our sample is genuinely more metal poor than $[\mathrm{Fe} / \mathrm{H}]=-1$. We do not find statistically significant evidence for a mass-metallicity relationship in either subpopulation, or in the sample as a whole, although this would be much better addressed with a larger sample. Even so, this is consistent with the results from larger M31 GC samples of Barmby et al. (2000) and Caldwell et al. (2011).

Observations of MW and M31 GCs show that the majority of old GCs have a roughly constant $M / L$ ratio (e.g., McLaughlin \& van der Marel 2005; Barmby et al. 2007; Strader et al. 2011). Under the assumption of constant $M / L$, relationships with integrated luminosity should reflect trends with GC mass. Velocity dispersion should also scale with GC mass according to the virial theorem.

In Figure 29 we examine the behavior of the $\alpha$-element ratios as a function of absolute $V$ magnitude $\left(M_{V}\right)$ and velocity dispersion $\left(v_{\sigma}\right)$, in order to investigate relationships of the abundance ratios with proxies for GC mass. It is interesting to look for trends with mass in order to gain insight on GC formation, particularly the role that gas retention may play in multiple populations or star-to-star abundance variations. As in Figure 28, in Figure 29, we show metal-poor and metalrich GCs with blue and red symbols. We also perform linear least-squares fits to the metal-poor, metal-rich, and whole sample, which we show with blue, red, and black dotted lines, respectively. Interestingly, we find positive correlations with luminosity and velocity dispersion for the $[\mathrm{Ca} / \mathrm{Fe}]$ and $[\mathrm{Si} / \mathrm{Fe}]$ in the metal-poor populations. The metal-rich subpopulation also shows correlations for $[\mathrm{Ca} / \mathrm{Fe}]$, but not for $[\mathrm{Si} / \mathrm{Fe}]$. For each relationship we also calculate the Spearman's rank correlation coefficient $(\rho)$ and the probability $(P)$ associated with the null hypothesis, which are noted in the upper left corner of each panel. The Spearman's rank calculation is most useful for the $[\mathrm{Ca} / \mathrm{Fe}]$ abundances, which have small, comparable errors for all of the GCs. The Spearman's rank calculation confirms a 

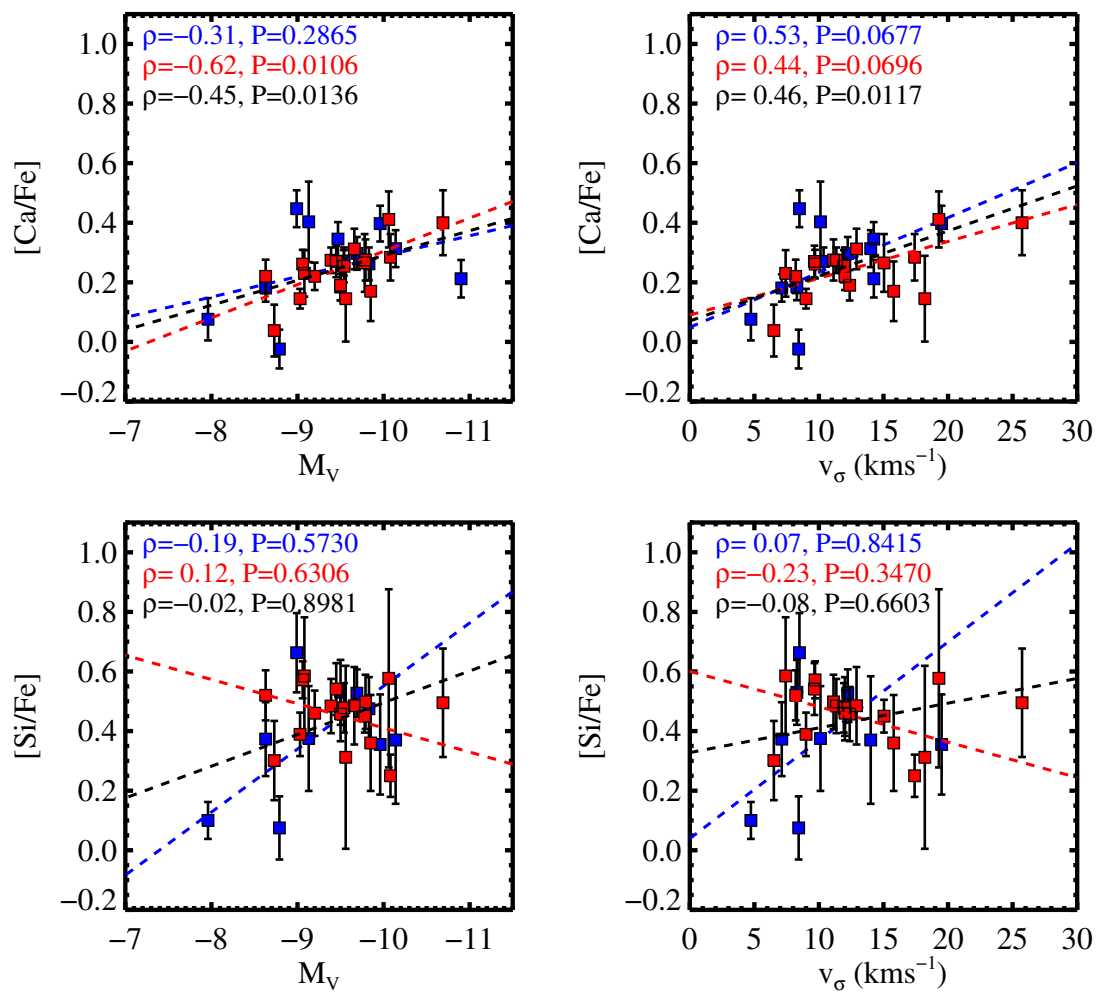

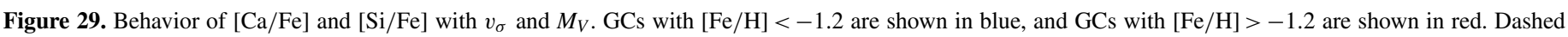

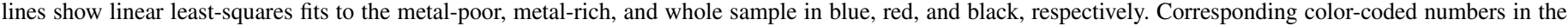
upper left corner of each panel show the Spearman rank correlation coefficient $(\rho)$ and the probability associated with the null hypothesis $(P)$.

(A color version of this figure is available in the online journal.)

moderate correlation with luminosity and velocity dispersion of the entire sample with $[\mathrm{Ca} / \mathrm{Fe}]$ to high probability.

We show the same analysis for the $[\mathrm{Ti}$ I/Fe] and [Ti II/Fe] abundances in Figure 30. Both ratios have a larger scatter with $M_{V}$ and $v_{\sigma}$, and while the trend in the metal-poor population is still present, it is much less pronounced. The Spearman's rank correlation calculation does not support a strong relationship of either $\mathrm{Ti}$ I or $\mathrm{Ti}$ II with these quantities.

The same analysis for the light element ratios is shown in Figure 31. We find more dramatic correlations, particularly for the metal-rich subpopulation for $[\mathrm{Mg} / \mathrm{Fe}],[\mathrm{Na} / \mathrm{Fe}]$, and possibly $[\mathrm{Al} / \mathrm{Fe}]$. Moreover, we find that the trends with luminosity and velocity dispersion for the metal-rich subpopulation are offset to higher abundance ratios than the metal-poor subpopulation in $[\mathrm{Mg} / \mathrm{Fe}]$, although we also note that the behavior of the metalpoor subpopulation is skewed by the three GCs with the lowest $[\mathrm{Mg} / \mathrm{Fe}]$, which span the entire range in GC mass. Accordingly, the Spearman's rank correlation coefficient is largest, with the smallest probability for the null hypothesis for the metal-rich $\mathrm{GCs}$ and $[\mathrm{Mg} / \mathrm{Fe}]$. The $[\mathrm{Na} / \mathrm{Fe}]$ abundances show more moderate correlations with luminosity and velocity dispersion. The $[\mathrm{Al} / \mathrm{Fe}]$ abundances also appear to correlate with luminosity and velocity dispersion, although we have fewer measurements for $\mathrm{Al} \mathrm{I}$, so the trends are less robust.

We also found trends of $[\mathrm{Na} / \mathrm{Fe}]$ and $[\mathrm{Al} / \mathrm{Fe}]$ with cluster mass for the LMC in $\mathrm{C} 12$, however in that work we could not determine if the correlations were driven primarily by the age or the mass of the clusters. In this case, our M31 GC sample is almost entirely old, so we do find that for old GCs in M31 the $[\mathrm{Na} / \mathrm{Fe}]$ and $[\mathrm{Mg} / \mathrm{Fe}]$ abundances correlate with proxies for cluster mass.
Our results and other recent GC IL work have interesting, although perhaps puzzling, implications for models of GC formation and GC self enrichment. A correlation of IL GC nitrogen abundances with mass was recently reported by Schiavon et al. (2013), for a larger sample of metal-rich $([\mathrm{Fe} / \mathrm{H}]>-1) \mathrm{M} 31$ GCs using low-resolution, high $\mathrm{S} / \mathrm{N}$ spectra and line indexes. However, Schiavon et al. (2013) did not find correlations of GC mass with $\mathrm{Mg}$ and $\mathrm{Ca}$, contrary to what we find here, but this could be because our high resolution measurements are more sensitive to small changes in the $\mathrm{Mg}$ and $\mathrm{Ca}$ features than the broader line indexes.

One question is whether abundance correlations with GC mass are related to multiple generations of star formation in GCs, and a second is whether the correlations give insight into the polluters that caused the present day star-to-star abundance variations in GCs. There are empirically motivated theoretical predictions for light element correlations with GC mass (Carretta et al. 2010b; Conroy 2012) that tie these two questions together. In particular, the models of Carretta et al. (2010b) and Conroy (2012) favor early AGB stars as the polluters that cause the $\mathrm{Na}-\mathrm{O}$ and $\mathrm{Mg}-\mathrm{Al}$ anticorrelations. Schiavon et al. (2013) propose that the nitrogen-mass correlation and lack of $\mathrm{Mg}$ - and Ca-mass correlations in their observations support AGB stars as the polluters (e.g., Renzini 2008; D'Ercole et al. 2012). The $\mathrm{Na}$ - and Al-mass correlations that we find here may also be consistent with an increased fraction of ejecta from AGB stars retained in more massive GCs. On the other hand, it is not clear that this would explain the $\mathrm{Mg}$ - and Ca-mass correlations that we find, since $\mathrm{Mg}$ is depleted in AGB stars and Ca should not be affected. However, we find the most extreme and lowest $[\mathrm{Mg} / \mathrm{Fe}]$ abundances in the metal-poor subpopulation only, 

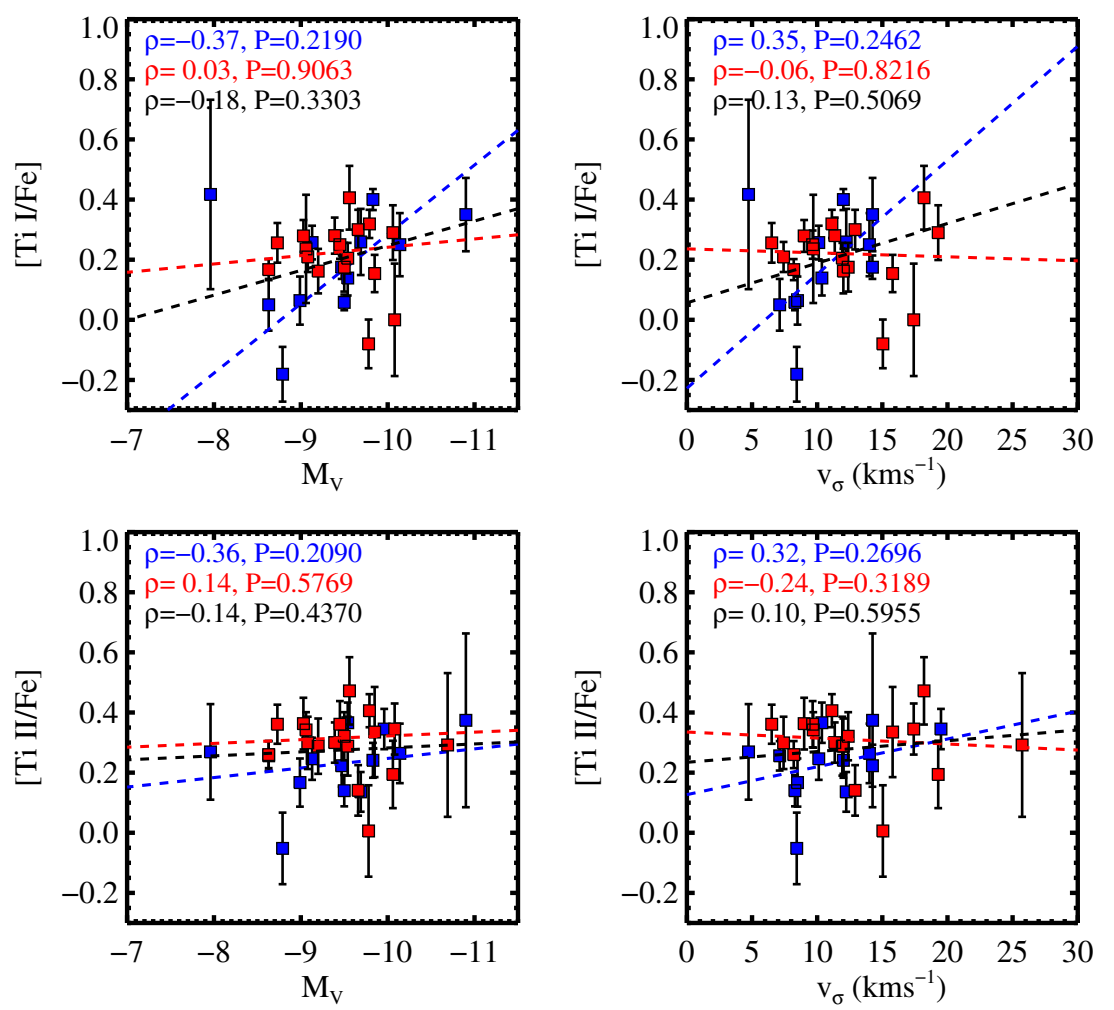

Figure 30. Same as Figure 29 for [Ti I/Fe] and [Ti II/Fe].

(A color version of this figure is available in the online journal.)

which do not seem to correlate with the proxies for GC mass. Therefore, the metal-poor subpopulation may still be consistent with self enrichment by AGB ejecta.

The $\mathrm{Mg}$-mass correlation in the metal-rich subpopulation may be indicative of a different form of self-enrichment, which may be more related to the $\mathrm{Ca}-$ mass correlation. The metal-rich subpopulation abundance correlations may instead be related to the self-enrichment scenario that forms the blue tilt, or mass-metallicity relationship, seen in the most massive GCs in early type galaxies. Further modeling is needed to determine how the abundance ratio trends we observe may be related to different GC self-enrichment scenarios.

\subsection{Behavior with Galactocentric Radius}

We show the $[\mathrm{Fe} / \mathrm{H}]$ behavior with galactocentric radius from M31 in Figure 32, using radii tabulated in the Bologna Catalog (Galleti et al. 2004). For context, we also show the larger sample of low resolution $[\mathrm{Fe} / \mathrm{H}]$ measurements of Caldwell et al. (2011), as well as the low resolution measurements of outer halo GCs from Alves-Brito et al. (2009). We find a spread in $[\mathrm{Fe} / \mathrm{H}]$ for GCs within $R_{\mathrm{M} 31} \sim 20 \mathrm{kpc}$, and a fairly constant $[\mathrm{Fe} / \mathrm{H}]$ for GCs at $R_{\mathrm{M} 31}>20 \mathrm{kpc}$. The mean value for the $5 \mathrm{GCs}$ in our sample that lie outside $20 \mathrm{kpc}$ from M31 is $[\mathrm{Fe} / \mathrm{H}]=$ $-1.63 \pm 0.10$, which is consistent with the metallicity of outer MW halo GCs of $[\mathrm{Fe} / \mathrm{H}]=-1.7$ (Harris 1996, 2010 revision).

A nearly constant $[\mathrm{Fe} / \mathrm{H}]$ for $\mathrm{M} 31$ outer halo GCs has also been found in previous works (Perrett et al. 2002; Mackey et al. 2007; Alves-Brito et al. 2009; Huxor et al. 2011). Like our analysis, Alves-Brito et al. (2009) found a flat metallicity distribution for GCs outside $30 \mathrm{kpc}$, with a mean metallicity of $[\mathrm{Fe} / \mathrm{H}]=-1.6$, which was estimated from line indexes. Using CMD-derived metallicities, (Huxor et al. 2011) found an outer halo metallicity of $[\mathrm{Fe} / \mathrm{H}] \sim-1.9$, which is lower than what we find, but the offset can probably be ascribed to a systematic offset between spectroscopic and photometric measurements.

Interestingly, Mackey et al. (2013) studied two newly discovered GCs from the PAndAS survey (Huxor et al. 2014), which have projected galactocentric radii of $\sim 85 \mathrm{kpc}$ from M31. From photometric CMD-based metallicities, Mackey et al. (2013) estimate that these GCs are both more metal-rich than other halo GCs, with $[\mathrm{Fe} / \mathrm{H}] \sim-1.35$, and possibly a few Gyr younger in age. However, these GCs are projected onto major stellar substructure in the M31 halo, and have radial velocities offset from the systemic velocity of M31, which makes it likely that these GCs were recently accreted into the M31 halo from a satellite galaxy. Mackey et al. (2013) suggest that these M31 GCs are analogs to the "young" MW halo GCs of Zinn (1993), which later studies proposed were accreted at late times (e.g., Da Costa \& Armandroff 1995).

Thus, evidence has been mounting that, like the MW, M31 has both a "young" and "old" halo, where the young GCs are likely GCs that were accreted late times and which may be younger and show chemical similarities to the dwarf galaxies in which they originated. The young and old halo GCs are likely related to the stream-like and smooth halo stellar populations, which are also associated with metal-rich $([\mathrm{Fe} / \mathrm{H}] \sim-0.7)$ and metalpoor $([\mathrm{Fe} / \mathrm{H}] \sim-1.5)$ abundances, respectively (Ibata et al. 2014). The old halo GCs appear to be uniformly old and metalpoor, consistent with the "proto-galactic" fragment formation scenario of old MW GCs first popularized by Searle \& Zinn (1978). Our current sample of M31 GCs with detailed chemical abundances appears to be drawn from the old halo population, in that all of our GCs at $R_{\mathrm{M} 31}>20 \mathrm{kpc}$ are old and metal-poor. A more complete high resolution census of the outer halo GCs will be interesting to study the younger metal-rich GCs at large projected radius to determine their detailed abundance patterns. 

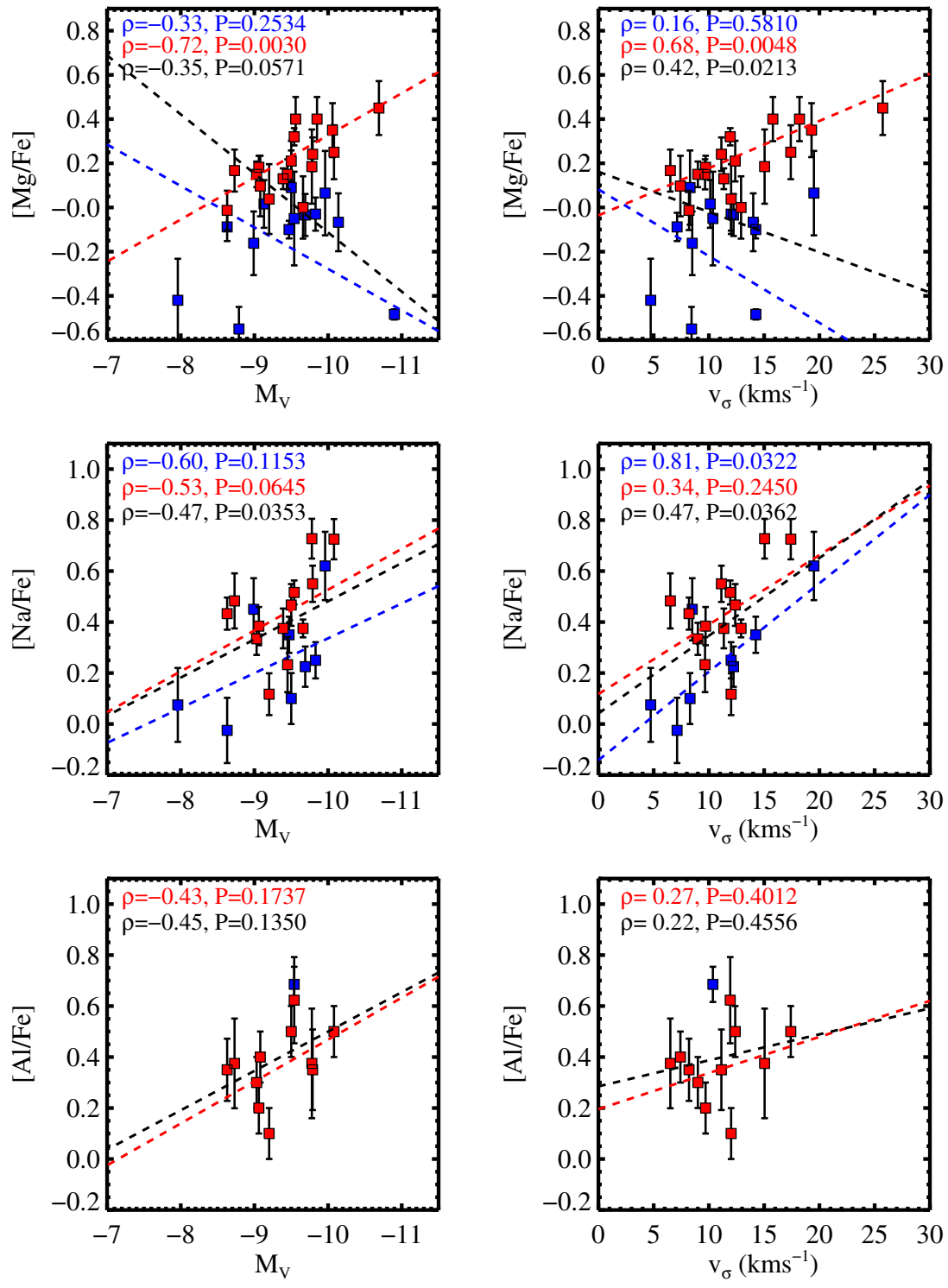

Figure 31. Same as Figure 29 for $[\mathrm{Mg} / \mathrm{Fe}]$, $[\mathrm{Na} / \mathrm{Fe}]$, and $[\mathrm{Al} / \mathrm{Fe}]$.

(A color version of this figure is available in the online journal.)

In addition to the behavior of $[\mathrm{Fe} / \mathrm{H}]$ with galactocentric radius, we are able to examine the behavior of the abundance ratios with radius, as shown for $[\mathrm{Ca} / \mathrm{Fe}],[\mathrm{Si} / \mathrm{Fe}],[\mathrm{Mg} / \mathrm{Fe}]$, and $[\mathrm{Na} / \mathrm{Fe}]$ in Figure 33. In the current sample, there are no compelling trends of the abundance ratios with radius, although it will be interesting to increase the sample size of GCs at large radii in the future in order to further investigate the chemical composition of the outer halo of M31.

\subsection{Star Formation and Accretion History of M31}

As presented in Section 4.3, we find that the $\alpha$-element abundances of this sample of M31 GCs strongly resemble the average properties of MW GCs and field stars. We find a plateau of $[\alpha / \mathrm{Fe}] \sim+0.3$ that extends from $[\mathrm{Fe} / \mathrm{H}]=-2$ to at least $[\mathrm{Fe} / \mathrm{H}]=-0.7$, which indicates that at early times the chemical enrichment of M31 was dominated by the ejecta of SN II. There is some indication from the $[\mathrm{Ca} / \mathrm{Fe}],[\mathrm{Si} / \mathrm{Fe}]$ and $[\mathrm{Ti} / \mathrm{Fe}]$ abundances that the highest metallicity GCs in our sample begin to decline at high $[\mathrm{Fe} / \mathrm{H}]$, which may indicate the increasing contribution of SN Ia enrichment when these GCs formed. Continued study of the highest metallicity GCs in M31 would be interesting to confirm this behavior.

A handful of the GCs in our sample have a scatter in the $[\alpha / \mathrm{Fe}]$ ratios obtained from different elements. This may indicate subtle differences in the ISM mixing when the M31 GCs were formed. At least one GC in our sample, G002, has a noticeably different $[\alpha / \mathrm{Fe}]$ enrichment pattern than the other GCs at similar $[\mathrm{Fe} / \mathrm{H}]$. In particular, the $\alpha$-element abundances of this cluster are lower than for other low-metallicity GCs $([\mathrm{Fe} / \mathrm{H}] \sim-1.6)$, which is similar to the situation of the MW GC Rup 106 (Brown et al. 1997; Villanova et al. 2013, [Fe/ $\mathrm{H}] \sim-1.5$ and $[\alpha / \mathrm{Fe}] \sim 0.0$. Because of the different $\alpha$-element abundance pattern, it is speculated that Rup 106 formed outside the MW and was later accreted. We compare the abundance ratios for Rup 106 from the recent analysis by Villanova et al. (2013) to both G002 and a more "halo-like" GC, B240, in Figure 34 for the elements in common ( $\mathrm{Fe}, \mathrm{Ca}, \mathrm{Si}, \mathrm{Ti} \mathrm{I}$, and $\mathrm{Mg}$ ). With the exception of $[\mathrm{Mg} / \mathrm{Fe}]$, it is clear that the abundance pattern of G002 closely resembles the abundance pattern of Rup 106, and neither Rup 106 nor G002 are similar 


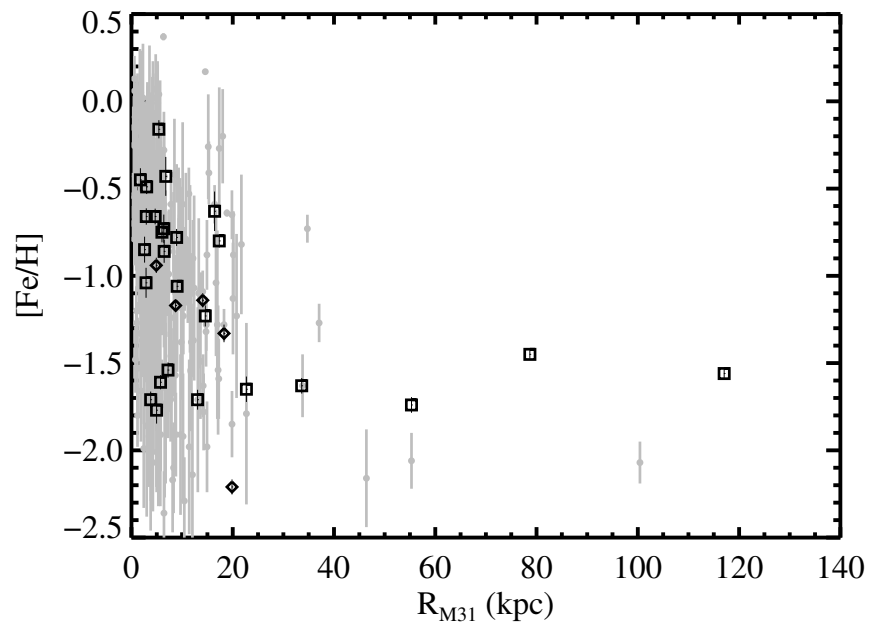

Figure 32. Behavior of $[\mathrm{Fe} / \mathrm{H}]$ with galactocentric radius from $\mathrm{M} 31\left(R_{\mathrm{M} 31}\right)$. Squares show the new M31 GCs from this work and diamonds show the GCs from $\mathrm{C} 09$. Gray points show the low-resolution spectra $[\mathrm{Fe} / \mathrm{H}]$ measurements of the larger sample of Caldwell et al. (2011), as well as the large radii GCs of Alves-Brito et al. (2009).

to $\mathrm{B} 240$. In general, lower $[\alpha / \mathrm{Fe}]$ abundances are found in dwarf galaxies than in the MW, although dwarf galaxies can also show a wide range in $[\alpha / \mathrm{Fe}]$ within themselves. For example, LMC GCs have mean $[\alpha / \mathrm{Fe}]$ ranging from +0.0 to +0.4 at $[\mathrm{Fe} / \mathrm{H}]<-1.5$ (Johnson et al. 2006; Mucciarelli et al. 2009, 2010; Colucci et al. 2012); Fornax GCs have $[\alpha / \mathrm{Fe}] \sim+0.15$ (Letarte et al. 2006), and the possible Sagittarius member clusters Terzan $8, \operatorname{Arp} 2$, and M54 have $[\alpha / \mathrm{Fe}]$ ranging from +0.2 to +0.4 (Mottini et al. 2008; Carretta et al. 2010a, 2014). We therefore suggest that G002 was likely accreted into the M31 GC system at late times from a massive dwarf galaxy. We will further investigate similarities of the abundances of
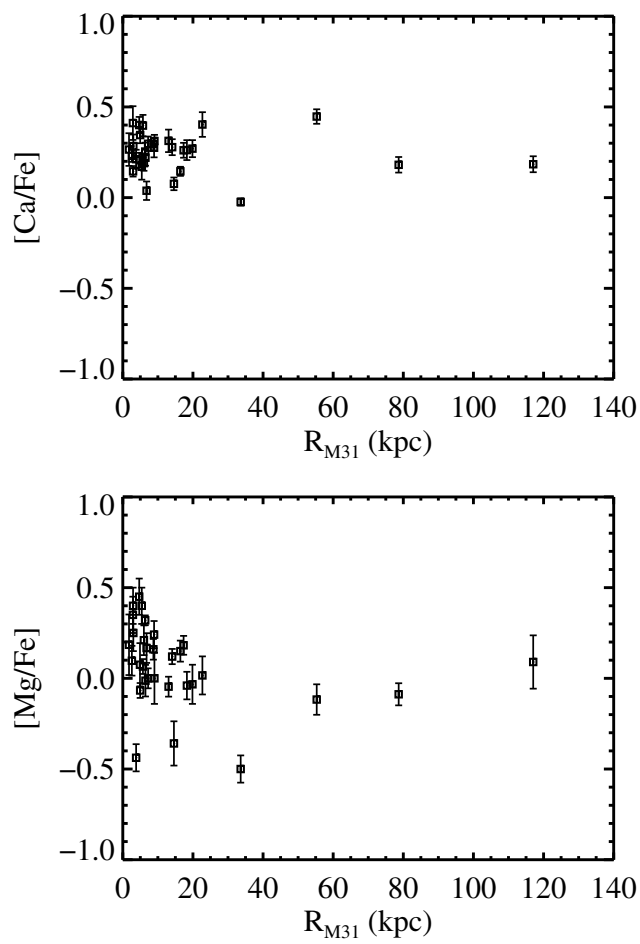

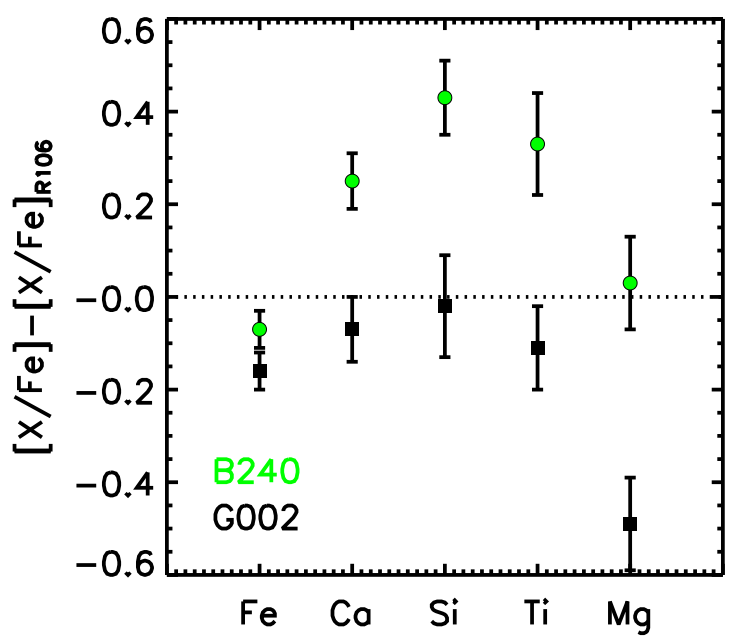

Figure 34. Comparison of the abundance pattern of G002 (black squares) and B240 (green circles) to that of Ruprecht 106 from Villanova et al. (2013). The abundance ratios $([\mathrm{X} / \mathrm{Fe}])$ are compared for the elements (X) shown, with the exception of $\mathrm{Fe}$, for which we show $[\mathrm{Fe} / \mathrm{H}]$. We have adjusted the abundances of Villanova et al. (2013) so that all abundance ratios use the same solar abundance pattern. For Ti, we compare our Ti I abundances, since the $[\mathrm{Ti} / \mathrm{Fe}]$ in Villanova et al. (2013) is measured solely from Ti I lines.

(A color version of this figure is available in the online journal.)

heavy elements in G002 with GC like Ruprecht 106 and those in dwarf galaxies in a future paper.

There is independent evidence that G002 is associated with a relic system. Mackey et al. (2010b) found that both G002 and G001 lie in an over density of GCs in the halo of M31, and classified the over density as a substructure named "Association 2." Follow-up velocity work on the M31 outer halo GC system was performed by Veljanoski et al. (2014), who found that the GCs in Association 2, including G002, fall into two distinct kinematic subsets. Their analysis showed a low probability
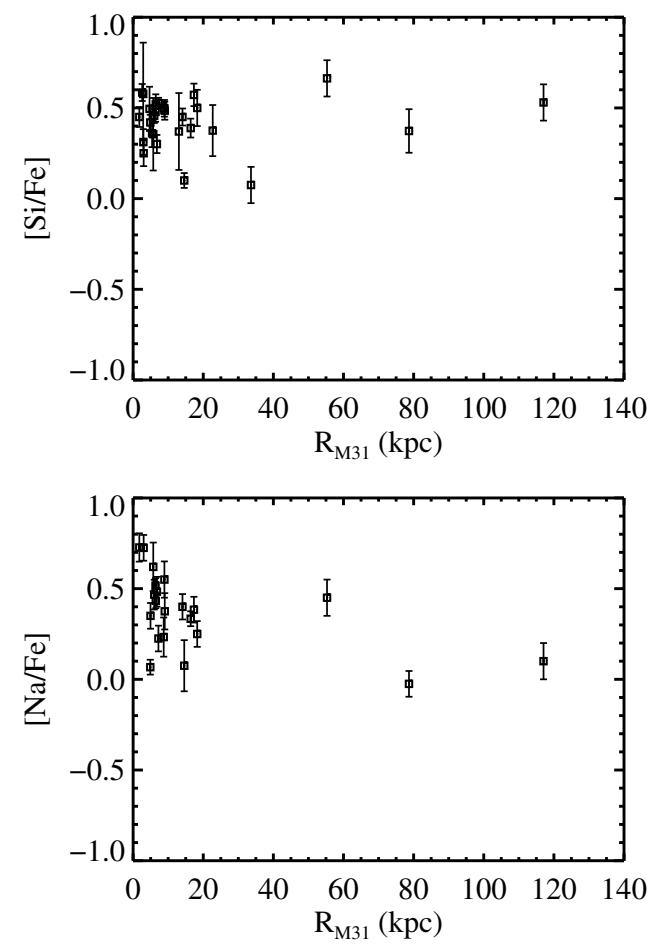

Figure 33. Behavior of abundance ratios $[\mathrm{Ca} / \mathrm{Fe}],[\mathrm{Si} / \mathrm{Fe}],[\mathrm{Mg} / \mathrm{Fe}]$, and $[\mathrm{Na} / \mathrm{Fe}]$ with galactocentric radius from $\mathrm{M} 31\left(R_{\mathrm{M} 31}\right)$. 

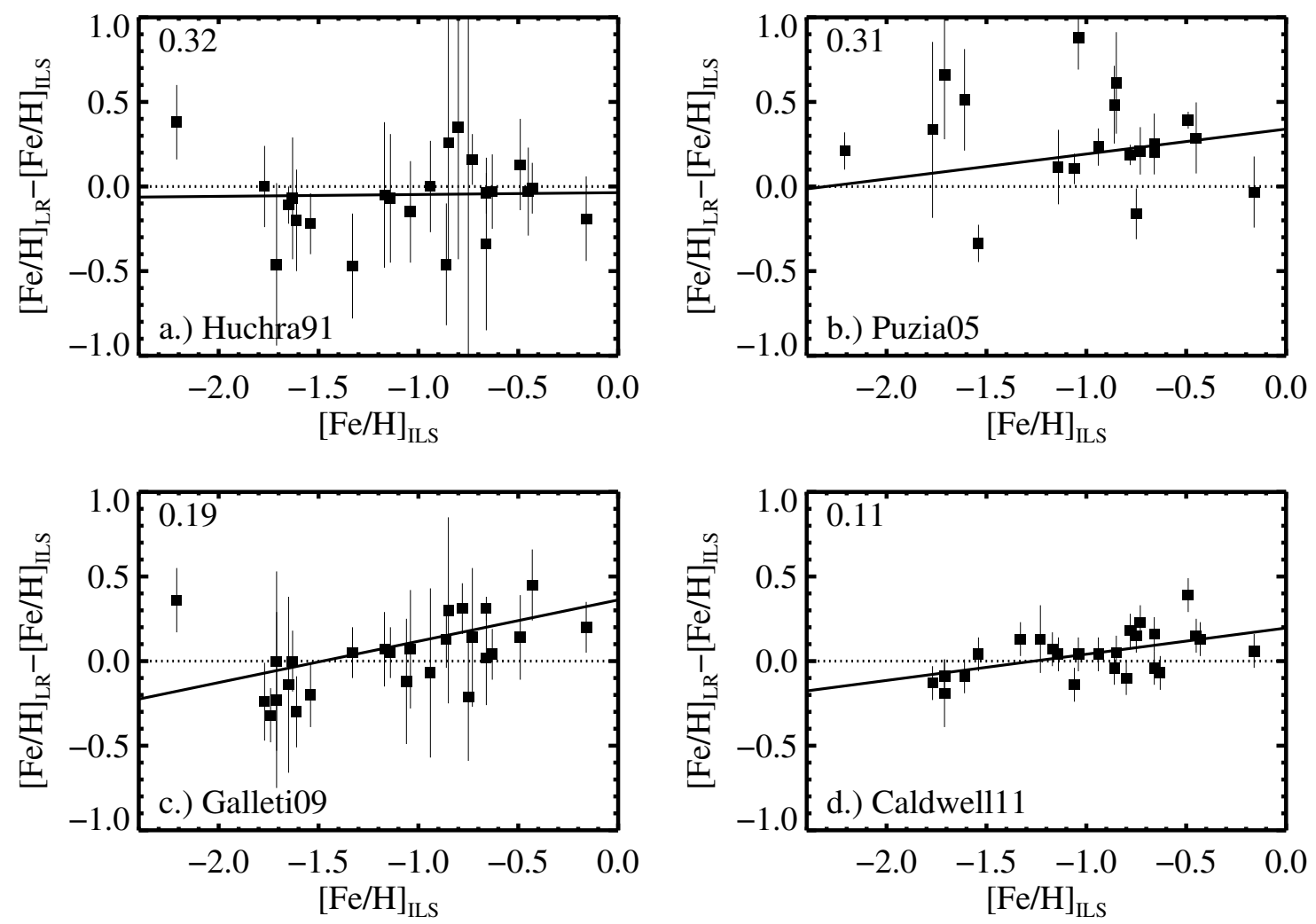

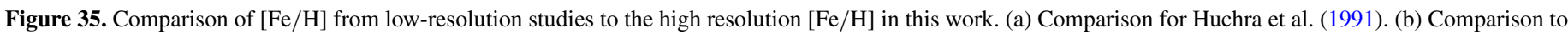

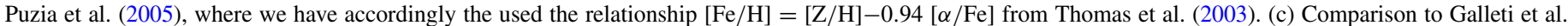

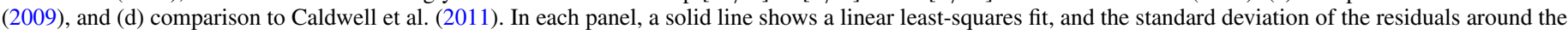
fit is noted in the upper left corner.

that the GCs in this region inhabit an over density and have distinct velocities by chance, and conclude that Association 2 may be a projection of two relic systems, possibly associated with the expected base of the prominent M31 substructure, the North-West Stream. According to Veljanoski et al. (2014) there are 10 GCs associated with Association 2, including G001, G002, H2, H7, H8, PAndAS-18, 19, 21, 22 and 23. Of these, G001, H2, H7, PAndAS-21, and PAndAS-22 are luminous enough $\left(M_{V}<-6\right)$ to be good candidates for high resolution ILS abundance analysis, and we therefore plan to target for follow-up. In the MW, metal-poor halo stars with low $[\mathrm{Ca} / \mathrm{Fe}]$ have been tagged as an "outlier" group (Cohen et al. 2013), so it would be extremely interesting if some or all of the GCs in Association 2 shared the abundance pattern of G002, and could be chemically tagged as a distinct accretion event in M31.

\subsection{Comparison with Previous Estimates: Fe and Alpha}

In this section, we compare our precise high resolution abundance and age measurements with the wealth of previous data on M31 GCs that have previously been obtained with lower resolution spectra and line index techniques.

First, we compare the high resolution $[\mathrm{Fe} / \mathrm{H}]$ to lowresolution $[\mathrm{Fe} / \mathrm{H}]$ in Figure 35 using measurements from Huchra et al. (1991), Puzia et al. (2005), Galleti et al. (2009), and Caldwell et al. (2011). It is important to note that "metallicity" measured from line index techniques isn't strictly an $[\mathrm{Fe} / \mathrm{H}]$ in the same sense as the high resolution $[\mathrm{Fe} / \mathrm{H}]$, which is measured directly from $\mathrm{Fe}$ lines. Instead, low-resolution estimates use a combination of metallicity sensitive indexes, which are largely sensitive to $\mathrm{Fe}$, but also to $\mathrm{Mg}$, for example (e.g., Burstein et al.
1984; Rose 1985; Brodie \& Huchra 1990; Worthey et al. 1994; Trager et al. 1998; Beasley et al. 2002; Thomas et al. 2003; Graves \& Schiavon 2008; Vazdekis et al. 2010; Thomas et al. 2011). Our goal in this work is not to identify the reasons why metallicity estimates vary between high resolution and low resolution (e.g., different stellar and atmospheric models, assumed stellar populations, or calibration samples), or vary between different low-resolution techniques themselves, since that level of detail is beyond the scope of the current work. Rather, we hope that our metallicity scale can be used for future calibration of low resolution techniques in abundance ranges that the MWs resolved GC system does not include.

Following the fit to the differences in Figure 35, it is clear that the low resolution solutions show a bias with metallicity that crosses over from under- to overestimating the true metallicity at about $[\mathrm{Fe} / \mathrm{H}]=-1.5$ to -1 . Figure 35 also shows that after accounting for the bias, the overall dispersion in the residuals is smallest for the Caldwell et al. (2011) sample, although it has more GCs that deviate by more than $1 \sigma$ because of the much smaller formal errors than in the other samples. While the claim of super-solar GCs in M31 has been around for some time (Huchra et al. 1991; Perrett et al. 2002; Puzia et al. 2005; Fan et al. 2008; Galleti et al. 2009; Caldwell et al. 2011), our IL spectra and analysis do not support that finding. Moreover, we note that the behavior seen in Figure 35 casts doubt on the likelihood that there are truly super-solar GCs in M31, as it is clear that abundances are overestimated in metal-rich clusters. A targeted follow up study of the presumed solar and supersolar GCs in M31 is necessary to determine if such clusters do exist; there are $\sim 10$ GCs in the Caldwell et al. (2011) sample 

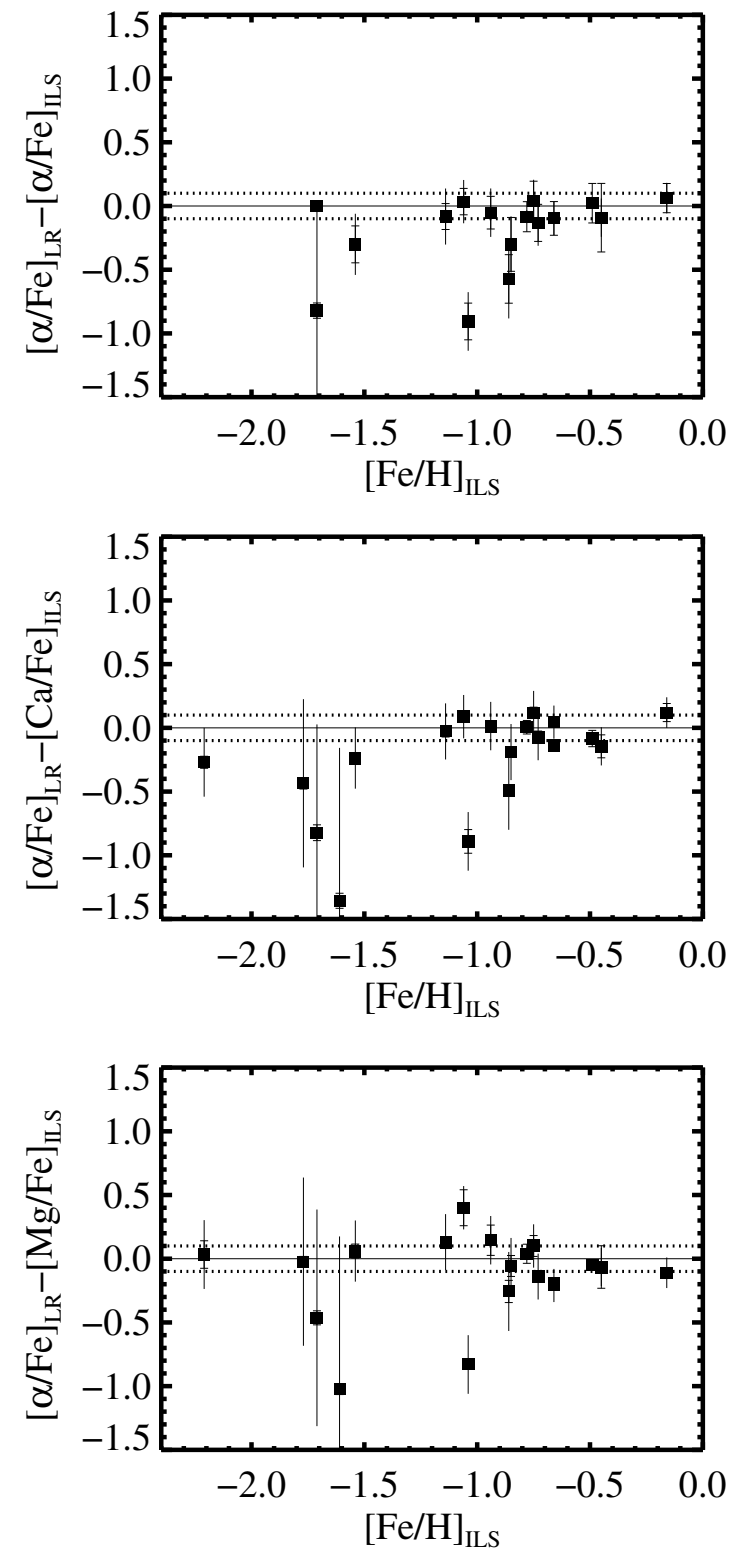

Figure 36. Comparison to the $[\alpha / \mathrm{Fe}]$ estimates of Puzia et al. (2005). The top panel compares the average of our $\mathrm{Ca}, \mathrm{Si}$, and $\mathrm{Ti}$ measurements, and the middle panel compares only to $\mathrm{Ca}$, which we have measured for all GCs in the sample. The bottom panel compares to $[\mathrm{Mg} / \mathrm{Fe}]$, which we note does not track the other $\alpha$ elements in the GCs. Dotted lines are shown at \pm 0.10 dex to guide the eye, which is approximately the limit for meaningful distinctions between "solar-scaled" and " $\alpha$-enhanced" abundances.

that have $[\mathrm{Fe} / \mathrm{H}]>0$ and are bright enough for IL abundance analysis $(V<18.5)$; however, the super-solar GCs tend to be projected onto the bulge and inner disk of M31, so the analysis of these GCs may be more difficult and special care would have to be taken for background subtraction.

While low-resolution bulk metallicity measurements are well established (and getting increasingly better according to Figure 35$),[\alpha / \mathrm{Fe}]$ estimates from low resolution have only started to become available recently and are prone to greater systematics and inconsistencies (see a longer discussion in Brodie $\&$ Strader 2006). With that in mind, we compare to the $[\alpha / \mathrm{Fe}]$ estimates from Puzia et al. (2005) in Figure 36, which are currently the only $[\alpha / \mathrm{Fe}]$ estimates that are explicitly tabulated for individual GCs (however we anticipate a much larger low resolu- tion $\alpha$-element sample will soon be available for comparison in R. P. Schiavon et al. 2015, in preparation). Therefore, while the $[\mathrm{Fe} / \mathrm{H}]$ comparison discussed above spanned several different line index models and techniques, we are only able to compare to one set of $[\alpha / \mathrm{Fe}]$ measurements in this case, which were made from the specific models of Thomas et al. (2003, 2004).

We compare both to the average of our $\mathrm{Ca}, \mathrm{Si}$, and $\mathrm{Ti}$ ratios, as well as the individual $\mathrm{Ca}$ ratios, and the individual $\mathrm{Mg}$ ratios. $\mathrm{A}$ range of \pm 0.10 dex is marked in Figure 36, which is effectively the minimum scatter for meaningfully distinguishing between solar-scaled ratios of $[\alpha / \mathrm{Fe}]=0.0$ and $\alpha$-enhanced ratios of $[\alpha / \mathrm{Fe}]=+0.3$.

Figure 36 suggests that high resolution is necessary for useful IL $[\alpha / \mathrm{Fe}]$ measurements (e.g., precisions $<0.1 \mathrm{dex}$ ), especially for metal poor GCs with $[\mathrm{Fe} / \mathrm{H}]<-1$. We find that low-resolution spectra can produce more accurate measurements of $[\alpha / \mathrm{Fe}]$ at higher metallicities, but that the accuracy quickly declines at metallicities below $[\mathrm{Fe} / \mathrm{H}] \sim-1.1$, at which $[\alpha / \mathrm{Fe}]$ is always underestimated. This can partly be explained by the degeneracy of the SSP models at lower metallicities and the unavoidable disappearance of the spectral lines, as can be seen in Puzia et al. (2005). However, we find that the low-resolution measurements appear to track the individual $[\mathrm{Mg} / \mathrm{Fe}]$ measurements better than the other $\alpha$ elements. This is not surprising, since strong $\mathrm{Mg}$ features probably dominate the low resolution $\alpha$ element indicators of the Thomas et al. (2003, 2004) models. However, it is a mixed blessing, since $\mathrm{Mg}$ does not necessarily track the true $\alpha$ element abundances in the GCs because of the effect of star-to-star abundance variations on the IL abundances. Ideally, a better low-resolution $\alpha$-element indicator would not be dominated by $\mathrm{Mg}$ features.

\section{SUMMARY}

We have presented analysis of high-resolution, high S/N, IL spectra of $31 \mathrm{GCs}$ in M31. We report precise radial velocities and velocity dispersions using our high quality data; velocity dispersions of nine of the GCs are measured here for the first time. We have presented refinements of our original technique for detailed abundance analysis of IL spectra of GCs, which we have used to obtain ages for the GCs and abundances of Fe from Fe I and Fe II lines, Ca I, Si I, Ti from Ti I and Ti II lines, Mg I, $\mathrm{Na}$, and $\mathrm{Al}$ I. Below we summarize the key results from this work.

1. For accurate age and abundance measurements, the spectra of clusters with large velocity dispersions $\left(v_{\sigma} \geqslant 15 \mathrm{~km} \mathrm{~s}^{-1}\right)$ and/or high metallicity $([\mathrm{Fe} / \mathrm{H}] \geqslant-0.3)$ must be analyzed using line synthesis in order to perform accurate continuum placement and to account for line blending.

2. Of the 31 GCs analyzed in this work, all of the GCs but B029 have ages consistent with being $\geqslant 10$ Gyr. For B029 we obtain an age of $\sim 2 \mathrm{Gyr}$, which is the first evidence for an intermediate GC in M31 that does not rely on integrated colors or Balmer line strengths.

3 The mean, low-metallicity $[\alpha / \mathrm{Fe}]$ plateau values of the M31 GCs in our sample are similar to the MW GC stellar abundances of Pritzl et al. (2005). Subtle differences in the overall patterns of individual $\alpha$ elements are seen, just as in the MW.

4. The abundances of the light elements $\mathrm{Mg}$ I, $\mathrm{Na}$, and $\mathrm{Al}$ I show indirect evidence for star-to-star abundance variations within the GCs, which was first hinted at in C09. 
5. We find correlations of $[\mathrm{Ca} / \mathrm{Fe}],[\mathrm{Na} / \mathrm{Fe}]$, and perhaps $[\mathrm{Al} / \mathrm{Fe}]$ with proxies for cluster mass $\left(M_{v}\right.$ and $\left.v_{\sigma}\right)$ when considering the sample as a whole, as well as when dividing the sample into metal-poor and metal-rich subpopulations. This is the first evidence of mass-metallicity relationships in elements other than Fe. We also find a strong correlation of $[\mathrm{Mg} / \mathrm{Fe}]$ with proxies for $\mathrm{GC}$ mass in the metalrich subpopulation. This may indicate that the mechanism responsible for star-to-star abundance variations in GCs in dependent on cluster mass.

6. We find at least one GC, G002, that has a significantly different $[\alpha / \mathrm{Fe}]$ abundance pattern than other GCs at similar $[\mathrm{Fe} / \mathrm{H}]$. This abundance pattern may indicate that this GC, which is also associated with the Association 2 overdensity, was accreted at late times into the M31 GC system.

7. We find a fairly constant low metallicity $([\mathrm{Fe} / \mathrm{H}]=-1.6)$ for the GCs in our sample that have projected galactocentric radii $>20 \mathrm{kpc}$ from M31. This resembles the "old" halo population in the MW.

Detailed abundances of additional Fe peak and heavy elements will be presented in the next papers in this series.

The authors thank the anonymous referee for a thoughtful report, which improved the clarity of the paper. J.E.C. is supported by an NSF Astronomy and Astrophysics Postdoctoral Fellowship under award AST-1302710. J.G.C. thanks NSF grant AST-0908139 for partial support. J.E.C. and R.A.B. thank NSF grant AST-0507350 for partial support. Digitized Sky Surveys were produced at the Space Telescope Science Institute under U.S. Government grant NAG W-2166. The Second Palomar Observatory Sky Survey (POSS-II) was made by the California Institute of Technology with funds from the National Science Foundation, the National Geographic Society, the Sloan Foundation, the Samuel Oschin Foundation, and the Eastman Kodak Corporation. The authors wish to recognize and acknowledge the very significant cultural role and reverence that the summit of Mauna Kea has always had within the indigenous Hawaiian community. We are most fortunate to have the opportunity to conduct observations from this mountain.

\section{REFERENCES}

Alves-Brito, A., Forbes, D. A., Mendel, J. T., Hau, G. K. T., \& Murphy, M. T. 2009, MNRAS, 395, L34

Asplund, M., Grevesse, N., Sauval, A. J., \& Scott, P. 2009, ARA\&A, 47, 481

Bai, G. S., Zhao, G., Chen, Y. Q., et al. 2004, A\&A, 425, 671

Bailin, J., \& Harris, W. E. 2009, ApJ, 695, 1082

Barklem, P. S., Christlieb, N., Beers, T. C., et al. 2005, A\&A, 439, 129

Barmby, P., \& Huchra, J. P. 2000, ApJL, 531, L29

Barmby, P., Huchra, J. P., Brodie, J. P., et al. 2000, AJ, 119, 727

Barmby, P., McLaughlin, D. E., Harris, W. E., Harris, G. L. H., \& Forbes, D. A. 2007, AJ, 133, 2764

Bastian, N., Lamers, H. J. G. L. M., de Mink, S. E., et al. 2013, MNRAS, 436, 2398

Beasley, M. A., Baugh, C. M., Forbes, D. A., Sharples, R. M., \& Frenk, C. S. 2002, MNRAS, 333, 383

Beasley, M. A., Brodie, J. P., Strader, J., et al. 2004, AJ, 128, 1623

Beasley, M. A., Brodie, J. P., Strader, J., et al. 2005, AJ, 129, 1412

Bernstein, R. A., \& McWilliam, A. 2005, in ASP Conf. Ser., Resolved Stellar

Populations, ed. D. Valls-Gabaud \& M. Chavez (San Francisco, CA: ASP)

Brodie, J. P., \& Huchra, J. P. 1990, ApJ, 362, 503

Brodie, J. P., \& Strader, J. 2006, ARA\&A, 44, 193

Brown, J. A., Wallerstein, G., \& Zucker, D. 1997, AJ, 114, 180

Brown, T. M., Ferguson, H. C., Smith, E., et al. 2004, ApJL, 613, L125

Burstein, D., Faber, S. M., Gaskell, C. M., \& Krumm, N. 1984, ApJ, 287, 586
Caldwell, N., Schiavon, R., Morrison, H., Rose, J. A., \& Harding, P. 2011, AJ, 141,61

Cameron, S. A. 2009, PhD thesis, Univ. Michigan

Carretta, E. 2006, AJ, 131, 1766

Carretta, E., Bragaglia, A., Gratton, R. G., et al. 2010a, A\&A, 520, A95

Carretta, E., Bragaglia, A., Gratton, R. G., et al. 2010b, A\&A, 516, A55

Carretta, E., Bragaglia, A., Gratton, R. G., et al. 2014, A\&A, 561, A87

Castelli, F., \& Kurucz, R. L. 2004, arXiv:astro-ph/0405087

Cohen, J. G. 2004, AJ, 127, 1545

Cohen, J. G., Christlieb, N., Thompson, I., et al. 2013, ApJ, 778, 56

Cohen, J. G., \& Kirby, E. N. 2012, ApJ, 760, 86

Colucci, J. E., \& Bernstein, R. A. 2012, ApJ, 749, 124

Colucci, J. E., Bernstein, R. A., Cameron, S. A., \& McWilliam, A. 2011, ApJ, 735,55

Colucci, J. E., Bernstein, R. A., Cameron, S. A., \& McWilliam, A. 2012, ApJ, 746, 29

Colucci, J. E., Bernstein, R. A., Cameron, S., McWilliam, A., \& Cohen, J. G. 2009, ApJ, 704, 385

Colucci, J. E., Bernstein, R. A., McWilliam, A., \& Cohen, J. G. 2013a, MmSAI, 84,50

Colucci, J. E., Fernanda Durán, M., Bernstein, R. A., \& McWilliam, A. 2013b, ApJL, 773, L36

Conroy, C. 2012, ApJ, 758, 21

Cordier, D., Pietrinferni, A., Cassisi, S., \& Salaris, M. 2007, AJ, 133, 468

Da Costa, G. S., \& Armandroff, T. E. 1995, AJ, 109, 2533

Decressin, T., Charbonnel, C., Siess, L., et al. 2009, A\&A, 505, 727

D'Ercole, A., D'Antona, F., Carini, R., Vesperini, E., \& Ventura, P. 2012, MNRAS, 423, 1521

Djorgovski, S. G., Gal, R. R., McCarthy, J. K., et al. 1997, ApJL, 474, L19

Dubath, P., \& Grillmair, C. J. 1997, A\&A, 321, 379

Dubath, P., Meylan, G., \& Mayor, M. 1997, A\&A, 324, 505

Eggen, O. J., Lynden-Bell, D., \& Sandage, A. R. 1962, ApJ, 136, 748

Fan, Z., Ma, J., de Grijs, R., \& Zhou, X. 2008, MNRAS, 385, 1973

Ferguson, J. W., Alexander, D. R., Allard, F., et al. 2005, ApJ, 623, 585

Forbes, D. A., \& Bridges, T. 2010, MNRAS, 404, 1203

Fulbright, J. P. 2000, AJ, 120, 1841

Fulbright, J. P., McWilliam, A., \& Rich, R. M. 2007, ApJ, 661, 1152

Galleti, S., Bellazzini, M., Buzzoni, A., Federici, L., \& Fusi Pecci, F. 2009, A\&A, 508,1285

Galleti, S., Federici, L., Bellazzini, M., Fusi Pecci, F., \& Macrina, S. 2004, A\&A, 416, 917

Goudfrooij, P., \& Kruijssen, J. M. D. 2014, ApJ, 780, 43

Gratton, R., Sneden, C., \& Carretta, E. 2004, ARA\&A, 42, 385

Graves, G. J., \& Schiavon, R. P. 2008, ApJS, 177, 446

Harris, W. E. 1996, AJ, 112, 1487

Harris, W. E. 2009, ApJ, 699, 254

Harris, W. E., Whitmore, B. C., Karakla, D., et al. 2006, ApJ, 636, 90

Huchra, J. P., Brodie, J. P., \& Kent, S. M. 1991, ApJ, 370, 495

Huxor, A. P., Ferguson, A. M. N., Tanvir, N. R., et al. 2011, MNRAS, 414,770

Huxor, A. P., Mackey, A. D., Ferguson, A. M. N., et al. 2014, MNRAS, 442,2165

Ibata, R. A., Lewis, G. F., McConnachie, A. W., et al. 2014, ApJ, 780, 128

Jiang, L., Ma, J., Zhou, X., et al. 2003, AJ, 125, 727

Johnson, J. A., Ivans, I. I., \& Stetson, P. B. 2006, ApJ, 640, 801

Kang, Y., Rey, S.-C., Bianchi, L., et al. 2012, ApJS, 199, 37

Kroupa, P. 2002, Sci, 295, 82

Larsen, S. S., Brodie, J. P., \& Strader, J. 2012, A\&A, 546, A53

Lee, H.-c., Lee, Y.-W., \& Gibson, B. K. 2002, AJ, 124, 2664

Lee, H.-c., \& Worthey, G. 2005, ApJS, 160, 176

Letarte, B., Hill, V., Jablonka, P., et al. 2006, A\&A, 453, 547

Ma, J., Wang, S., Wu, Z., et al. 2012, AJ, 143, 29

Mackey, A. D., Ferguson, A. M. N., Irwin, M. J., et al. 2010a, MNRAS, 401, 533

Mackey, A. D., Huxor, A., Ferguson, A. M. N., et al. 2007, ApJL, 655, L85

Mackey, A. D., Huxor, A. P., Ferguson, A. M. N., et al. 2010b, ApJL, 717, L11

Mackey, A. D., Huxor, A. P., Ferguson, A. M. N., et al. 2013, MNRAS, 429, 281

Maxwell, A. J., Wadsley, J., Couchman, H. M. P., \& Sills, A. 2014, MNRAS, 439, 2043

McConnachie, A. W., Irwin, M. J., Ferguson, A. M. N., et al. 2005, MNRAS, 356,979

McConnachie, A. W., Irwin, M. J., Ibata, R. A., et al. 2009, Natur, 461, 66

McLaughlin, D. E., \& van der Marel, R. P. 2005, ApJS, 161, 304

McWilliam, A. 1998, AJ, 115, 1640

McWilliam, A., \& Bernstein, R. A. 2008, ApJ, 684, 326 
McWilliam, A., Preston, G. W., Sneden, C., \& Searle, L. 1995a, AJ, 109, 2757 McWilliam, A., Preston, G. W., Sneden, C., \& Shectman, S. 1995b, AJ, 109,2736

McWilliam, A., \& Rich, R. M. 1994, ApJS, 91, 749

Mieske, S., Jordán, A., Côté, P., et al. 2010, ApJ, 710, 1672

Milone, A. P., Bedin, L. R., Piotto, G., \& Anderson, J. 2009, A\&A, 497, 755

Mottini, M., Wallerstein, G., \& McWilliam, A. 2008, AJ, 136, 614

Mucciarelli, A., Origlia, L., \& Ferraro, F. R. 2010, ApJ, 717, 277

Mucciarelli, A., Origlia, L., Ferraro, F. R., \& Pancino, E. 2009, ApJL, 695, L134

Peacock, M. B., Maccarone, T. J., Knigge, C., et al. 2010, MNRAS, 402, 803

Percival, S. M., \& Salaris, M. 2011, MNRAS, 412, 2445

Perrett, K. M., Bridges, T. J., Hanes, D. A., et al. 2002, AJ, 123, 2490

Pietrinferni, A., Cassisi, S., Salaris, M., \& Castelli, F. 2004, ApJ, 612, 168

Pietrinferni, A., Cassisi, S., Salaris, M., \& Castelli, F. 2006, ApJ, 642, 797

Piotto, G. 2009, in IAU Symp. 258, The Ages of Stars, ed. E. E. Mamajek, D.

R. Soderblom, \& R. F. G. Wyse (Cambridge: Cambridge Univ. Press), 233

Pritzl, B. J., Venn, K. A., \& Irwin, M. 2005, AJ, 130, 2140

Puzia, T. H., Perrett, K. M., \& Bridges, T. J. 2005, A\&A, 434, 909

Reddy, B. E., Lambert, D. L., \& Allende Prieto, C. 2006, MNRAS, 367, 1329

Renzini, A. 2008, MNRAS, 391, 354

Rey, S.-C., Sohn, S. T., Beasley, M. A., et al. 2009, ApJL, 700, L11

Rose, J. A. 1985, AJ, 90, 1927

Sakari, C. M., Shetrone, M., Venn, K., McWilliam, A., \& Dotter, A. 2013, MNRAS, 434, 358

Sakari, C. M., Venn, K., Shetrone, M., Dotter, A., \& Mackey, D. 2014, MNRAS, 443,2285

Sbordone, L., Bonifacio, P., Marconi, G., Buonanno, R., \& Zaggia, S. 2005, A\&A, 437, 905

Schiavon, R. P., Caldwell, N., Conroy, C., et al. 2013, ApJL, 776, L7
Schiavon, R. P., Rose, J. A., Courteau, S., \& MacArthur, L. A. 2004, ApJL, 608, L33

Searle, L., \& Zinn, R. 1978, ApJ, 225, 357

Sneden, C. 1973, ApJ, 184, 839

Strader, J., Caldwell, N., \& Seth, A. C. 2011, AJ, 142, 8

Strader, J., \& Smith, G. H. 2008, AJ, 136, 1828

Thomas, D., Johansson, J., \& Maraston, C. 2011, MNRAS, 412, 2199

Thomas, D., Maraston, C., \& Bender, R. 2003, MNRAS, 339, 897

Thomas, D., Maraston, C., \& Korn, A. 2004, MNRAS, 351, L19

Tonry, J., \& Davis, M. 1979, AJ, 84, 1511

Trager, S. C., Worthey, G., Faber, S. M., Burstein, D., \& González, J. J. 1998, ApJS, 116, 1

Vazdekis, A., Sánchez-Blázquez, P., Falcón-Barroso, J., et al. 2010, MNRAS, 404, 1639

Veljanoski, J., Mackey, A. D., Ferguson, A. M. N., et al. 2014, MNRAS, 442, 2929

Venn, K. A., Irwin, M., Shetrone, M. D., et al. 2004, AJ, 128, 1177

Villanova, S., Geisler, D., Carraro, G., Moni Bidin, C., \& Muñoz, C. 2013, ApJ, 778,186

Vogt, S. S., Allen, S. L., Bigelow, B. C., et al. 1994, Proc. SPIE, 2198, 362

Wang, S., \& Ma, J. 2013, AJ, 146, 20

Woosley, S. E., \& Weaver, T. A. 1995, ApJS, 101, 181

Worthey, G. 1994, ApJS, 95, 107

Worthey, G., Faber, S. M., Gonzalez, J. J., \& Burstein, D. 1994, ApJS, 94, 687

Zaritsky, D., Colucci, J. E., Pessev, P. M., Bernstein, R. A., \& Chandar, R. 2012, ApJ, 761, 93

Zaritsky, D., Colucci, J. E., Pessev, P. M., Bernstein, R. A., \& Chandar, R. 2013, ApJ, 770, 121

Zinn, R. 1993, in ASP Conf. Ser. 48, The Globular Cluster-Galaxy Connection, ed. G. H. Smith \& J. P. Brodie (San Francisco, CA: ASP), 38 